División de Ciencias Básicas e Ingeniería
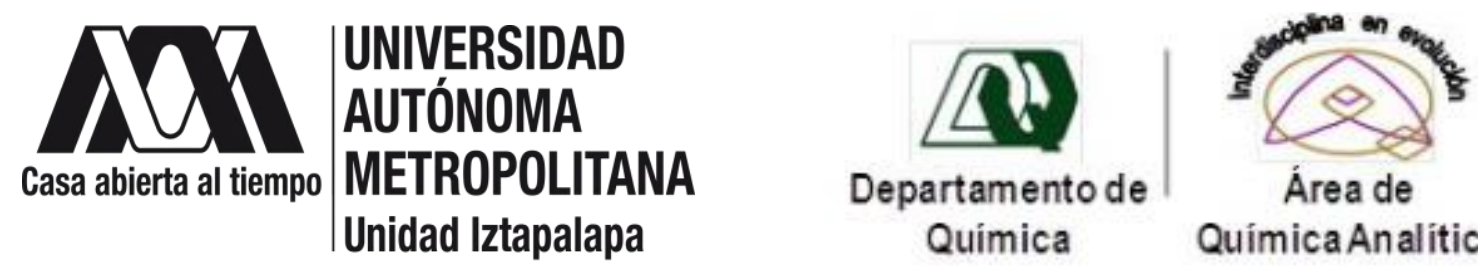

Química Analítica

\title{
ESTUDIO DE LA ESTABILIDAD QUÍMICA DE LA CURCUMINA EN MEDIO ACUOSO: FORMACIÓN DE COMPUESTOS SUPRAMOLECULARES CON $\beta$-CICLODEXTRINA.
}

TESIS

QUE PARA OBTENER EL GRADO DE

Doctor en Ciencias (Química)

\author{
PRESENTA \\ Q. Jorge Martínez Guerra
}

Directores de Tesis:

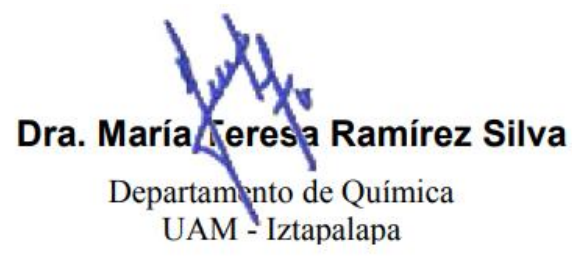

Departampnto de Química

UAM - Iztapalapa

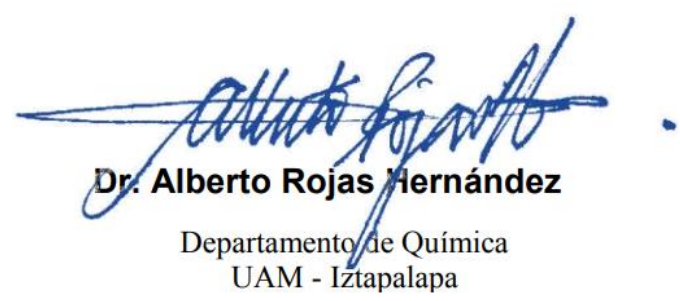

\section{Jurado Evaluador:}

Dra. Gabriela Roa Morales

Ciencias Ambientales, Facultad de Química UAEMex

Presidente
Dra. Silvia Corona Avendaño

Departamento de Materiales UAM - Azcapotzalco
Secretario
Dr. Carlos Andrés Galán Vidal

Centro de Investigaciones Químicas Instituto de Ciencias Básicas e Ingeniería, UAEH

$$
\begin{gathered}
\text { Laboratorio de Química Ambiental } \\
\text { Centro Conjunto de Investigación } \\
\text { Química, UAEMex } \\
\text { Vocal }
\end{gathered}
$$

Dr. Jorge Iván Aldana González

Departamento de Materiales UAM - Azcapotzalco Vocal

Ciudad de México, 23 de Marzo de 2020. 
"Si no sabes a dónde vas, cualquier camino te conduce allí" - Lewis Carroll 


\section{DEDICATORIAS:}

A mis padres y abuelita Claudia.

A Oswaldo Frausto (Ozwa) mi más sincero agradecimiento, amor y cariño.

"Investigar significa pagar la entrada por adelantado y entrar sin saber lo que se va a ver"

- Robert Oppenheimer 


\section{AGRADECIMIENTOS:}

A la Universidad Autónoma Metropolitana Unidad Iztapalapa por estos años de crecimiento profesional, académico e incluso personal en mí camino hacia la obtención del Doctorado.

Al Consejo Nacional de Ciencia y Tecnología (CONACYT) por la beca brindada (No. 306024) durante la realización de mi proyecto de investigación.

A la Dra. María Teresa Ramírez Silva (Tere) porque no sólo fue un pilar importante en la dirección de este trabajo, sino además ha sido una gran amiga donde su cubículo en muchas mañanas se convertía prácticamente en un saloncito para terapia expandiendo mi mente no solo en la Química sino en este camino que llamamos vida. Tu calidad humana es inigualable, ¡Gracias por todas tus enseñanzas y apoyarme cuando más lo he necesitado! y ¡Gracias por dejarme ser ahora un hijo académico más tuyo!.

Al Dr. Alberto Rojas Hernández por aceptarme desde un inicio en el laboratorio y ayudarme a satisfacer mi sed de conocimiento en la Química Analítica. Es un modelo a seguir.

A la Dra. Silvia Corona, por dejarme conocer el gran ser humano que eres con esa disciplina inquebrantable que te caracteriza tanto al momento de trabajar. Gracias por acompañarme en este camino desde mis primeras evaluaciones y ser testigo de mi evolución y culminación de este trabajo. ¡Tus aportes fueron valiosos y cruciales!.

Al Dr. Manuel Palomar por esas calurosas pláticas durante la realización de mi primer artículo. ¡Debatir contigo ha sido una de las mejores experiencias en mi formación profesional y personal!.

Al Dr. Mario Romero Romo porque además de un gran profesionista es un gran ser humano. ¡Gracias por tus aportes!.

Al Dr. Carlos Galán Vidal dado que su perspectiva ayudó a enriquecer positivamente la versión final de este trabajo.

Gracias a la Dra. Patricia Balderas, Dra. Gabriela Roa y Dr. Iván Aldana.

A mis compañeros y colegas: Dafne, Julio Alva, Julio Vega, Karina, Marlene, Adriana, Alzu, Lucero, Miguel, Yuri, Cristal, Fernando y Selene (UAM-A).

Al Q.F.B. Oswaldo Frausto González (Ozwa) porque expandiste mi mente de una manera que nunca imaginé. Gracias por todos los momentos y anécdotas que hemos construido y que guardo con todo mi cariño en mi corazón y por dejarme ver en ti la gran calidad de ser humano que eres: tus altos valores y tu esencia cautivaron mi mente y corazón. Me contagiaste de tu curiosidad y amor por la naturaleza y saber cómo funciona el mundo biológico. Nunca olvidaré los debates cada que podíamos acerca de mis experimentos. Muchas ideas que aportaste se llevaron a la práctica experimental. ¡Gracias por creer, motivar y contribuir al desarrollo de mi potencial!. Mi cariño, amor y estima te acompañen siempre. 


\section{ESTUDIO DE LA ESTABILIDAD QUÍMICA DE LA CURCUMINA EN MEDIO ACUOSO: FORMACIÓN DE COMPUESTOS SUPRAMOLECULARES CON $\beta$-CICLODEXTRINA.}




\section{ÍNDICE}

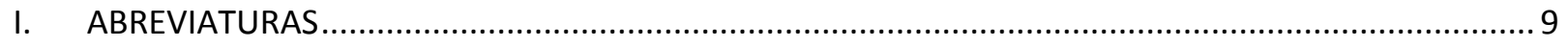

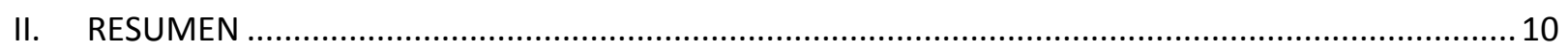

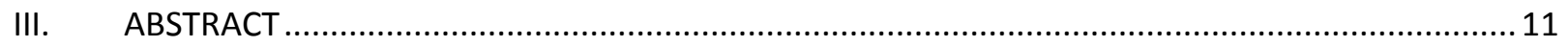

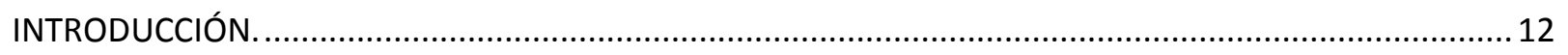

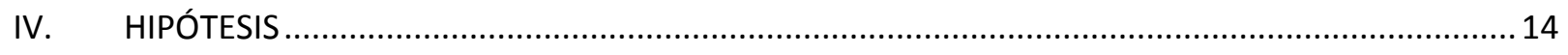

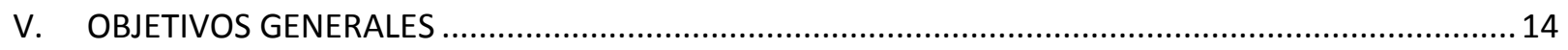

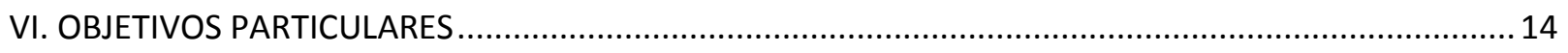

CAPÍTULO 1. Estudio de estabilidad de la curcumina por espectrofotometría de UV-Vis y por

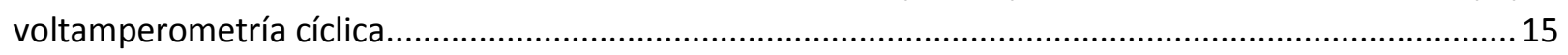

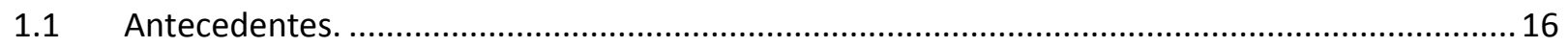

1.2 Estudio preliminar de la estabilidad de la curcumina en medio acuoso.......................................17

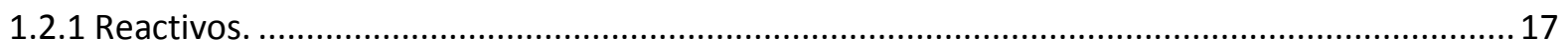

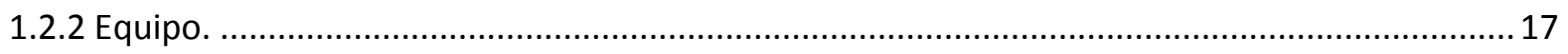

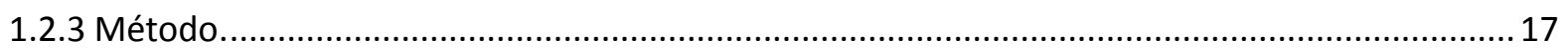

1.2.4 Influencia de las condiciones experimentales sobre la estabilidad de la curcumina por

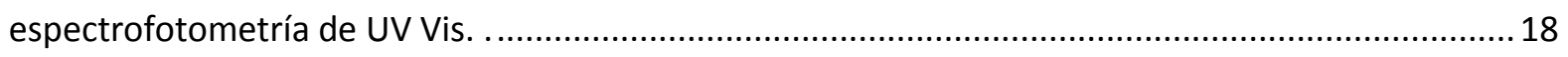

1.3 Estudio de la estabilidad de la curcumina en medio acuoso. Cálculo de la velocidad de

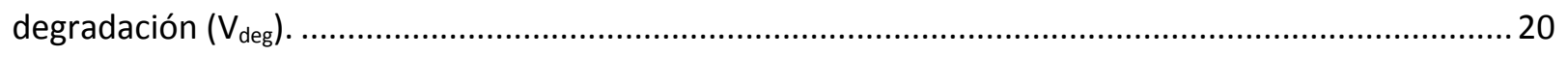

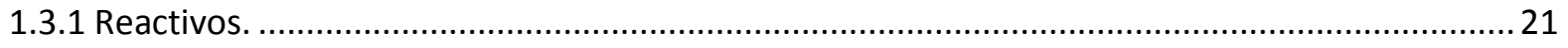

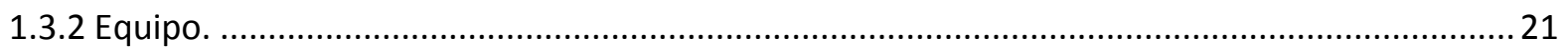

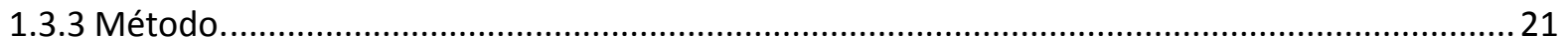

1.3.4 Monitoreo de la estabilidad de la curcumina por espectrofotometría de UV-Vis.......................22

1.3.5 Monitoreo de la estabilidad de la curcumina por voltamperometría cíclica................................25

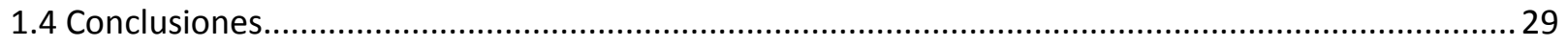

CAPÍTULO 2. Determinación de las constantes de acidez de la curcumina por espectrofotometría de

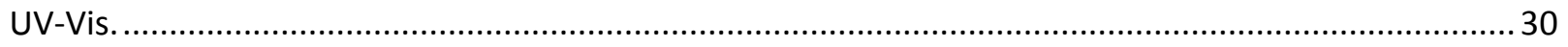

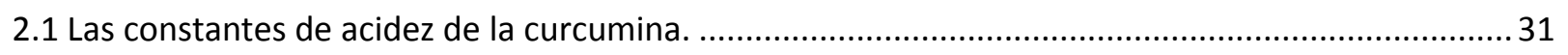

2.2 Determinación de las constantes de acidez de la curcumina bajo condiciones experimentales

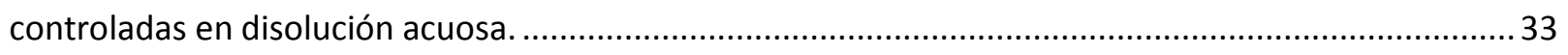

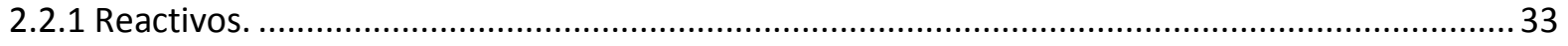


2.2.4 Cálculo de las constantes de acidez utilizando una estrategia gráfica y el programa

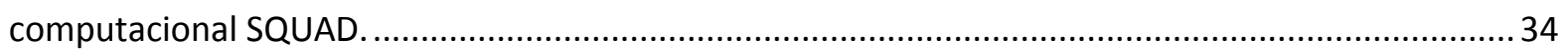

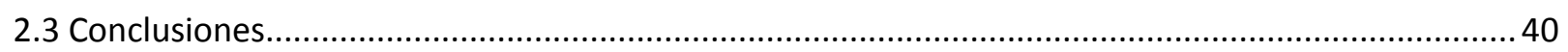

CAPÍTULO 3. Estrategias de estabilización para la Curcumina en medio acuoso. .................................... 41

3.1 Estabilización química de la curcumina mediante la formación de un compuesto de coordinación

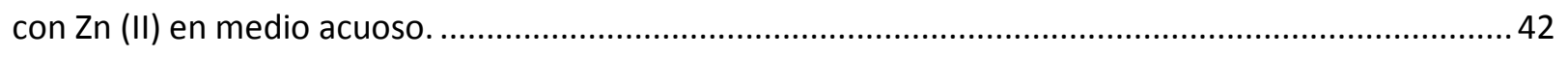

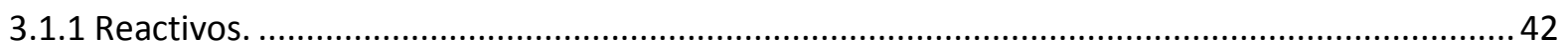

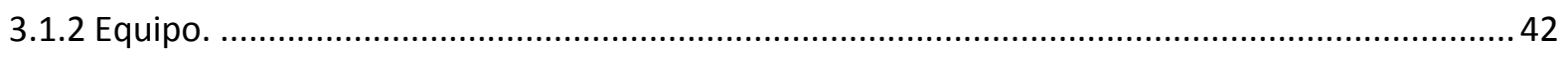

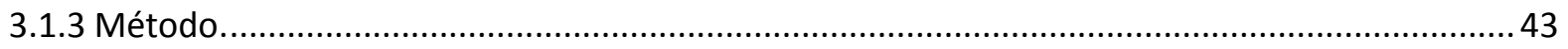

3.1.4 Estudio espectro-electroquímico de la interacción Curcumina-Zn (II) a pH $3.576 \pm 0.001$........ 43

3.1.5 Estudio espectro-electroquímico de la interacción Curcumina-Zn (II) a pH $7.025 \pm 0.001$........ 45

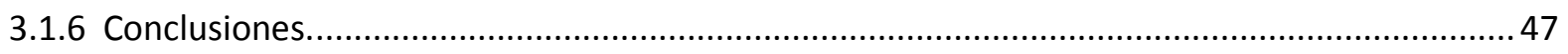

3.2 Estabilización química de la curcumina mediante la formación de un compuesto de inclusión con $\beta$ -

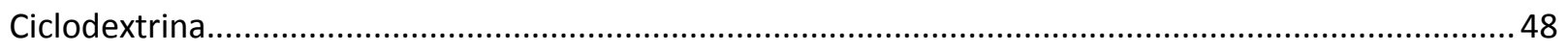

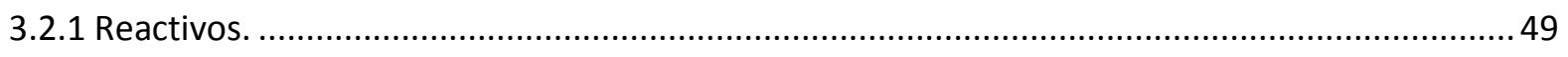

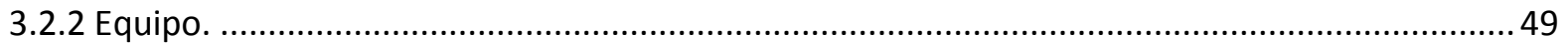

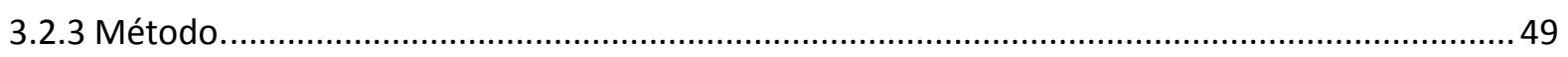

3.2.4 Estudio de la influencia de las condiciones experimentales sobre la estabilidad de la curcumina en medio acuoso en presencia de diferentes concentraciones de $\beta$-CD por espectrofotometría de

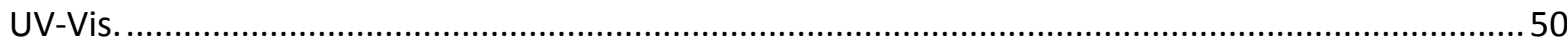

3.2.5 Estudio de la influencia de las condiciones experimentales sobre la estabilidad de la curcumina en medio acuoso por voltamperometría cíclica en medio amortiguado de $\mathrm{p} \beta-\mathrm{CD}=2$.

3.3 Determinación por espectrofotometría de UV-Vis de la constante del complejo de inclusión

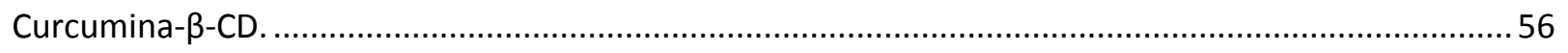

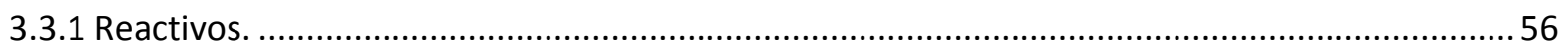

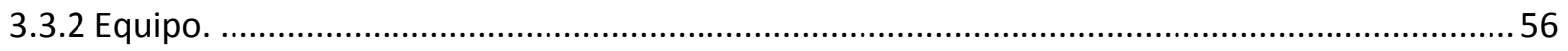

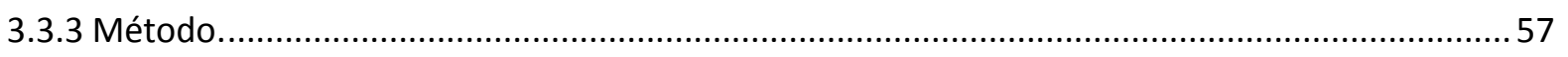

3.3.4 Cálculo de las constantes de inclusión de la curcumina-ß-Ciclodextrina en medio ácido..........57

3.3.5 Cálculo de la constante de inclusión de la curcumina- $\beta$-Ciclodextrina en medio básico. ...........61

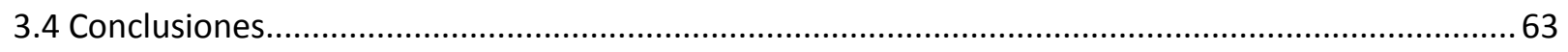


CAPÍTULO 4. Método analítico para la cuantificación de Curcumina en disolución acuosa en presencia de $\beta$-Ciclodextrina.

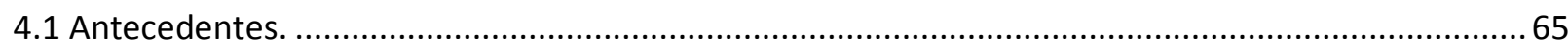

4.2 Diseño de un método analítico para la cuantificación de curcumina en medio amortiguado de $\beta$-CD

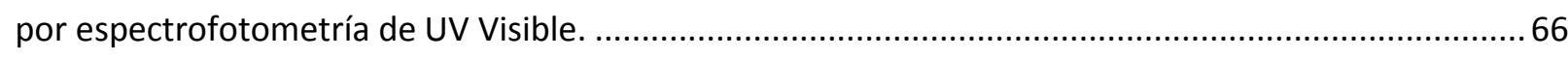

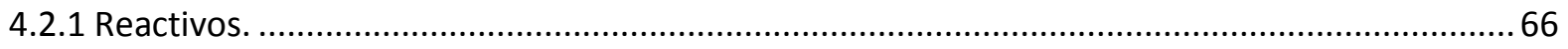

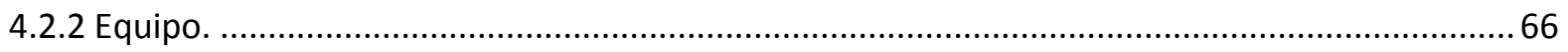

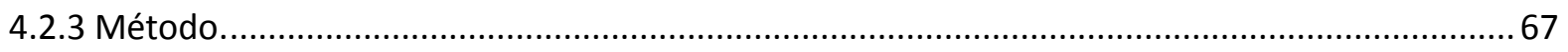

4.2.4 Cálculo de los parámetros analíticos de desempeño del método de cuantificación...................67

4.3 Diseño de un método analítico para la cuantificación de curcumina en medio amortiguado de $\beta$-CD por voltamperometría cíclica. ..................................................................................................... 70

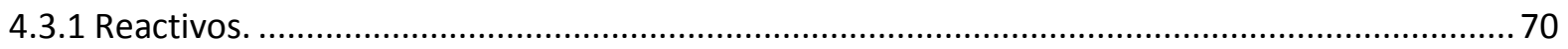

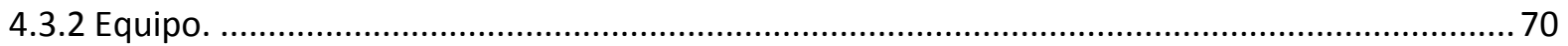

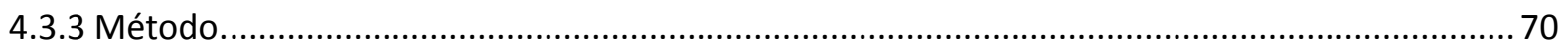

4.3.4 Cálculo de los parámetros analíticos de desempeño del método de cuantificación...................71

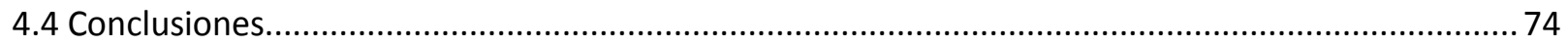

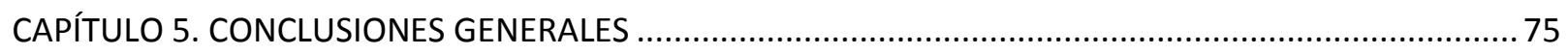

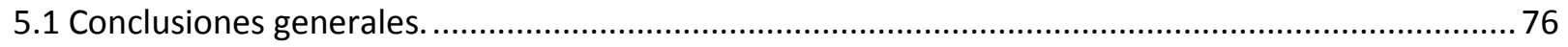

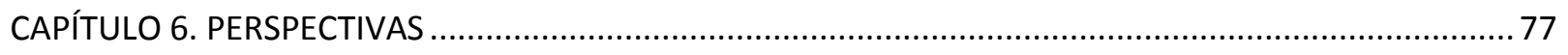

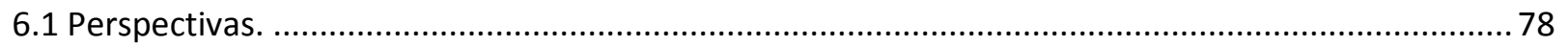

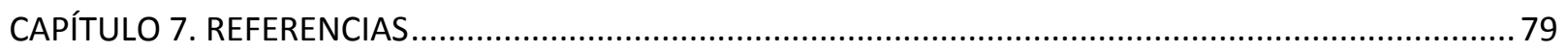

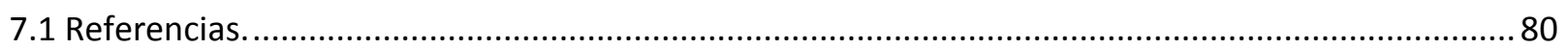

APÉNDICE 1 Estabilidad de la solución stock de curcumina [0.002 M] en medio acuoso .......................82

APÉNDICE 2 Estudio $E_{\lambda}$ y propuesta del mecanismo de oxidación de la curcumina en medio acuoso....84

APÉNDICE 3 Stability QUotiens from Absorbance Data (SQUAD) .................................................... 86

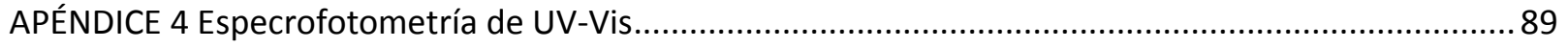

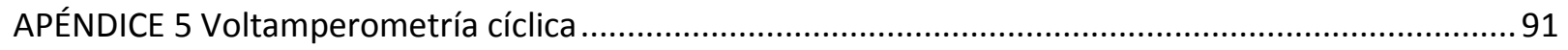

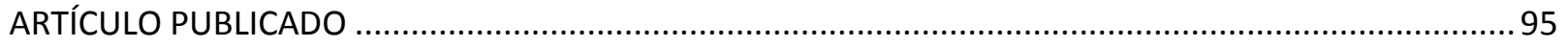

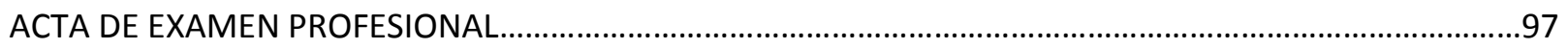




\section{ABREVIATURAS}

Cur

$\mathrm{H}_{3} \mathrm{Cur}$

$\mathrm{H}_{2} \mathrm{Cur}^{-}$

HCur ${ }^{2-}$

Cur ${ }^{3-}$

$\beta-C D$

$p \beta-C D$

$\mathrm{N}_{2}$ (g)

$\mathrm{NaOH}$

$\lambda$

$\mathrm{nm}$

UV-Vis

$\mathrm{mM}$

$\mu \mathrm{A}$

SQUAD

$\sigma$

U

$\mathrm{HClO}_{4}$

EPC

ER

EA

$\mu \mathrm{M}$

$\mathrm{Ip}^{\mathrm{a}}$

Ip $\mathrm{p}^{\mathrm{c}}$

$E p^{a}$

$E p^{c}$

\% DC
Curcumina

Especie de curcumina totalmente protonada

Especie de curcumina biprotonada

Especie de curcumina monoprotonada

Especie de curcumina totalmente desprotonada

Beta ciclodextrina

Operador matemático que representa el menos logaritmo base 10 de la concentración de beta ciclodextrina

Nitrógeno gaseoso

Hidróxido de sodio

Longitud de onda

nanómetros

Ultravioleta-Visible

milimolar

microampere

Stability QUotiens from Absorbance Data

Desviación estándar

Suma de los residuos de absorbancia cuadráticos

Ácido perclórico

Electrodo de Pasta de Carbono

Electrodo de Referencia

Electrodo Auxiliar o contraelectrodo

micromolar

Corriente de pico anódico

Corriente de pico catódico

Potencial de pico anódico

Potencial de pico catódico

Porcentaje de degradación de la curcumina 


\section{RESUMEN}

En este trabajo se estudia el impacto de diferentes variables experimentales sobre la estabilidad de la curcumina evaluando el impacto que tienen sobre la respuesta de espectrofotométrica y voltamperométrica. A partir de esta información se hace un estudio de estabilidad obteniendo valores de porcentaje de degradación así como valores de velocidad de degradación de la curcumina en medio acuoso a diferentes valores de $\mathrm{pH}$ bajo dos condiciones experimentales: a) sin control experimental y b) bajo condiciones controladas. Siendo este último definido por: la protección de las disoluciones de trabajo de la luz incidente, ausencia de $\mathrm{O}_{2(\mathrm{~g})}$ mediante el burbujeo con gas $\mathrm{N}_{2}(\mathrm{~g})$ así como una atmósfera inerte con este mismo gas y una temperatura constante $(21.0 \pm 0.1){ }^{\circ} \mathrm{C}$. Conociendo esta información, se propone un método para calcular las constantes de acidez de la curcumina en medio acuoso.

Adicionalmente se exploran dos estrategias para disminuir la velocidad de degradación de la curcumina en medio acuoso mediante: 1) la formación de compuestos de coordinación entre la curcumina y el zinc y 2) la formación de compuestos supramoleculares entre la curcumina y la $\beta$-ciclodextrina siendo esta última la que mejores resultados ofrece.

A partir de esto se propone el diseño de un método analítico de cuantificación en medio acuoso bajo condiciones experimentales controladas en medio amortiguado de $\beta$-ciclodextrina y $\mathrm{pH}$. 


\section{ABSTRACT}

In this work, the impact of different experimental variables on the stability of curcumin is studied by evaluating the impact they have on the spectrophotometric and voltammetric response. Based on this information, a stability study is obtained, obtaining degradation percentage values as well as degradation rate values of curcumin in aqueous medium at different $\mathrm{pH}$ values under two experimental conditions: a) without experimental control and b) under controlled conditions. The latter being defined by: the protection of working solutions from incident light, absence of $\mathrm{O}_{2(\mathrm{~g})}$ by bubbling with gas $\mathrm{N}_{2(\mathrm{~g})}$ as well as an inert atmosphere with this same gas and a constant temperature $(21.0 \pm 0.1)^{\circ} \mathrm{C}$. Knowing this information, a method is proposed to calculate the acidity constants of curcumin in aqueous medium.

Additionally, two strategies to reduce the degradation rate of curcumin in aqueous medium are explored by: 1) the formation of coordination compounds between curcumin and zinc and 2) the formation of supramolecular compounds between curcumin and $\beta$-cyclodextrin being the latter offers the best results.

From this, the design of an analytical method of quantification in aqueous medium is proposed under controlled experimental conditions in buffer medium of $\beta$ cyclodextrin and $\mathrm{pH}$. 


\section{INTRODUCCIÓN.}




\section{INTRODUCCIÓN.}

La curcumina [1,7-bis(4-hidroxi-3-metoxifenol)-1,6-heptadieno-3,5-diona] es el principal compuesto de la curcumina turmérica, comúnmente llamada cúrcuma. Sin embargo, también está presente pero en menor proporción e importancia biológica la demetoxicurcumina, la bisdemetoxicurcumina y la ciclocurcumina [1].

Su presentación turmérica es la de un sólido amarillo que se puede encontrar en los rizomas de la planta Curcuma Longa L. la cual forma parte de la familia de las zingiberáceas nativa del suroeste de la India [1].

Se sabe que la cúrcuma se ha utilizado desde tiempos muy antiguos como especia, sobre todo en la comida asiática, además se le ha atribuido propiedades antiinflamatorias (desde una perspectiva empírica) y se ha usado como un auxiliar en problemas estomacales. Según la Food, Drug \& Administration, FDA, por sus siglas en inglés; la curcumina no es tóxica.

Hoy en día, está reportado ampliamente que la curcumina es un compuesto fenólico que presenta propiedades antiinflamatorias [1,2], antioxidantes [1, 3-7], anticancerígenas [1, 6-8] y antivirales [1, 9-13] por mencionar las más importantes.

Sin embargo, su baja solubilidad en agua y su pobre biodisponibilidad ha frenado su potencial aplicación en la industria farmacéutica.

En la Figura 1 se muestra la estructura química de la curcumina la cual presenta un equilibrio tautomérico ceto-enol, siendo la forma enólica la de mayor predominio en solución acuosa con un $99.5 \%$ de abundancia [14, 15].<smiles>COc1cc(/C=C/C(=O)CC(=O)/C=C/c2ccc(O)c(OC)c2)ccc1O</smiles><smiles>COc1cc(/C=C/C(=O)/C=C(O)/C=C/c2ccc(O)c(OC)c2)ccc1O</smiles>

Figura 1. Equilibrio ceto-enol de la curcumina. 


\section{HIPÓTESIS}

Conocer las diferentes variables que influyen sobre la estabilidad química de la curcumina permitirá controlar el medio de reacción para obtener respuestas confiables y reproducibles durante su caracterización y su determinación analítica en medio acuoso.

\section{OBJETIVOS GENERALES}

Determinar las condiciones experimentales que influyen sobre la degradación química de la curcumina en medio acuoso así como diseñar estrategias de estabilización eficientes que permitan su caracterización y cuantificación en medio acuoso.

\section{OBJETIVOS PARTICULARES}

a) Calcular la velocidad de degradación de la curcumina en medio acuoso mediante espectrofotometría de UV-Vis y voltamperometría cíclica a tres diferentes valores de $\mathrm{pH}: 3.576,7.025$ y 10.526 .

b) Determinar las constantes de acidez de la curcumina en medio acuoso mediante espectrofotometría de UV- Vis.

c) Desarrollar una estrategia para optimizar la estabilidad química de la curcumina en disolución acuosa mediante la formación de compuestos de coordinación con el Zinc (II)

d) Estudiar la estabilidad de la curcumina en disolución acuosa mediante la formación de compuestos supramoleculares con la $\beta$-ciclodextrina.

e) Calcular las constantes de inclusión en disolución acuosa entre la curcumina y la $\beta$-ciclodextrina por espectrofotometría de UV-Vis.

f) Desarrollar un método analítico de cuantificación para la curcumina en medio amortiguado de $\beta$-ciclodextrina por espectrofotometría de UV-Vis y voltamperometría cíclica. 


\section{CAPÍTULO 1. Estudio de estabilidad de la curcumina por espectrofotometría de UV-Vis y por voltamperometría cíclica.}

"No estudio por saber más, sino por ignorar menos" 


\subsection{Antecedentes.}

Está reportado que las disoluciones acuosas de curcumina presentan procesos de degradación. Tonnesen y Karlsen [16] ponen en evidencia la degradación de curcumina en el intervalo de $\mathrm{pH}$ de 7 a 10 encontrando que el $\mathrm{pH}$ debe mantenerse por debajo de 7 para garantizar su estabilidad. Sin embargo, analizando el método descrito por los autores, la reacción de degradación de la curcumina la detienen adicionando $\mathrm{HCl}$ hasta $\mathrm{pH} 2$ inyectando su muestra a un sistema cromatográfico determinando que la vainillina, el ácido ferúlico y el feruloilmetano son los principales productos de degradación [17]. Wang et al. [18] mostraron evidencia sobre la degradación de curcumina en diferentes sistemas buffer (citrato, fosfatos y carbonatos) calculando una cinética de degradación de primer orden a $37^{\circ} \mathrm{C}$ determinando además que la estabilidad de la curcumina en medio acuoso es mayor conforme el valor de $\mathrm{pH}$ es más ácido presentando un tiempo de vida media, $\mathrm{t}_{1 / 2}$, de hasta 118.63 minutos reportando los mismos productos de degradación que Tonnesen y Karlsen [16,17]. Sin embargo, es importante mencionar que en el método descrito por Wang et al. [18] amortiguan el nivel de acidez hasta $\mathrm{pH} 3$ de las disoluciones de trabajo con $\mathrm{HCl} 6 \mathrm{~N}$ previo al análisis cromatográfico.

Recientemente, Gordon et al. [19] proponen que la curcumina en disolución acuosa ( $\mathrm{pH}$ 7.4) se autooxida a diferentes especies entre ellas las que reportan Tonnesen y Karlsen $[16,17]$ y Wang et. al. [18] pero comentan que la vainillina, el ácido ferúlico y el feruloilmetano son solo productos minoritarios y que el producto principal de degradación es la biciclopentadiona.

Bernabé et al. [20] encuentran que la curcumina en medio básico presenta un máximo de absorción a $468 \mathrm{~nm}$ el cual va decreciendo conforme el pH se vuelve más ácido. Tang et al. [21] reportan el mismo comportamiento pero además reportan que conforme el pH se vuelve más ácido aparece una nueva banda de transición a 360 $\mathrm{nm}$. Dicha banda también es reportada por Balasubramanian [22] a $343 \mathrm{~nm}$ la cual es asociada a una transición electrónica $\pi$ a $\pi^{*}$ aplicando un modelo de energía de orbitales moleculares para la especie neutra basado en el hamiltoniano de PariserParr-Pople (PPP).

Dada la diferencia en el $\mathrm{pH}$ inicial de la disoluciones de curcumina entre los trabajos de Tonnesen y Karlsen [16,17] y Wang et al. [18]; así como las discrepancias en el comportamiento espectrofotométrico entre Bernabé et al. [20], Tang et al. [21] y Balasubramanian [22]; existe la posibilidad que la curcumina en medio ácido no sea del todo estable. Muchos autores han tomado como válidos los resultados sobre la estabilidad de la curcumina en $\mathrm{pH}$ ácidos [21, 23-24] para hacer estudios sobre diferentes aspectos de la curcumina tales como: la determinación de sus constantes de inclusión con $\beta$-ciclodextrina [21], propiedades antioxidantes, como potencial neuroprotector [23] e incluso su cuantificación [24], por lo tanto, en este trabajo consideramos muy importante volver a estudiar la estabilidad de la curcumina en medio acuoso. 


\subsection{Estudio preliminar de la estabilidad de la curcumina en medio acuoso.}

Está reportado que algunas moléculas orgánicas presentan procesos de degradación en agua los cuales dependen directamente de las condiciones experimentales, por ejemplo; el nivel de acidez, de la intensidad de luz incidente a las disoluciones de trabajo, presencia de oxígeno, $\mathrm{O}_{2(g)}$, disuelto en el sistema que pueda contribuir a la oxidación de la molécula bajo estudio, por mencionar solo los más importantes [25-27].

A continuación se presenta el estudio de cómo influye el control de estas condiciones experimentales sobre la respuesta de la curcumina por espectrofotometría de UV-Vis.

\subsubsection{Reactivos.}

Curcumina grado analítico marca Sigma Aldrich ( $\geq 99.5 \%)$ el cual se utilizó sin ningún proceso de purificación, lentejas de $\mathrm{NaOH}$ marca Macron, $\mathrm{HClO}_{4}$ concentrado

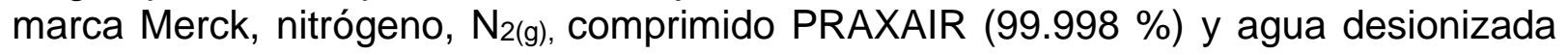
(18.2 M $\Omega$ ) libre de materia orgánica obtenida con un filtro PURE-LAB Plus UV.

\subsubsection{Equipo.}

La lectura de $\mathrm{pH}$ se realiza con un potenciómetro marca sensION equipado con un electrodo de vidrio de $\mathrm{Ag}^{\circ} \mid \mathrm{AgCl}[\mathrm{KCl}, 3 \mathrm{M}]$ marca Hach $5014 \mathrm{~T}$.

Para el control de la temperatura se utiliza un recirculador Grant W14.

Cada espectro de absorción se registra mediante un espectrofotómetro Lambda 20 UV Vis equipado con celdas de cuarzo $1 \mathrm{~cm}$ de longitud de paso óptico. El intervalo de adquisición de los espectros es de 200 a $700 \mathrm{~nm}$ a una velocidad de 1960 $\mathrm{nm} \mathrm{min}^{-1}$.

\subsubsection{Método.}

Monitoreo de la estabilidad de la curcumina por espectrofotometría de UV-Vis. Se monitorea la respuesta de absorbancia a tiempo cero de una disolución de trabajo de curcumina [32.5 $\mu \mathrm{M}]$ proveniente de una solución stock [0.002 M] la cual es previamente preparada en $\mathrm{NaOH}$ [5 mM] (esta se mantiene en refrigeración, ver Apéndice 1) a tres diferentes valores de pH: a) $\mathrm{pH} 3.576 \pm 0.001$, b) $\mathrm{pH} 7.025 \pm$ 
0.001 y c) $\mathrm{pH} 10.526 \pm 0.001$. Estos registros se hacen de manera independiente y bajo diferentes condiciones experimentales:

a) Sin ningún control experimental sobre el sistema de trabajo.

b) Trabajando en ausencia de luz.

c) Burbujeando previamente la disolución con $\mathrm{N}_{2(\mathrm{~g})}\left(1 \mathrm{~min} \mathrm{~mL}^{-1}\right)$ manteniendo una atmósfera con este mismo gas durante el tiempo que dura el experimento.

d) Temperatura constante de $(21.0 \pm 0.1){ }^{\circ} \mathrm{C}$ durante el tiempo que dura el experimento.

e) Manteniendo el control de todas estas variables experimentales al mismo tiempo.

Es importante mencionar que para estudiar cada valor de $\mathrm{pH}$ y cada condición experimental se hace utilizando disoluciones de trabajo de curcumina recién preparadas.

1.2.4 Influencia de las condiciones experimentales sobre la estabilidad de la curcumina por espectrofotometría de UV Vis. .

En la Figura 2 se muestran los espectros de absorción iniciales (tiempo 0 min) de la curcumina [32.5 $\mu \mathrm{M}$ ] bajo las diferentes condiciones experimentales expuestas en el punto 1.2.3. En la Figura 2a se muestran los espectros de absorción a pH 3.576 \pm 0.001 , en la Figura $2 \mathrm{~b}$ son los espectros de absorción a pH $7.025 \pm 0.001$ mientras que los de pH $10.526 \pm 0.001$ se muestran en la Figura $2 \mathrm{c}$.

a)

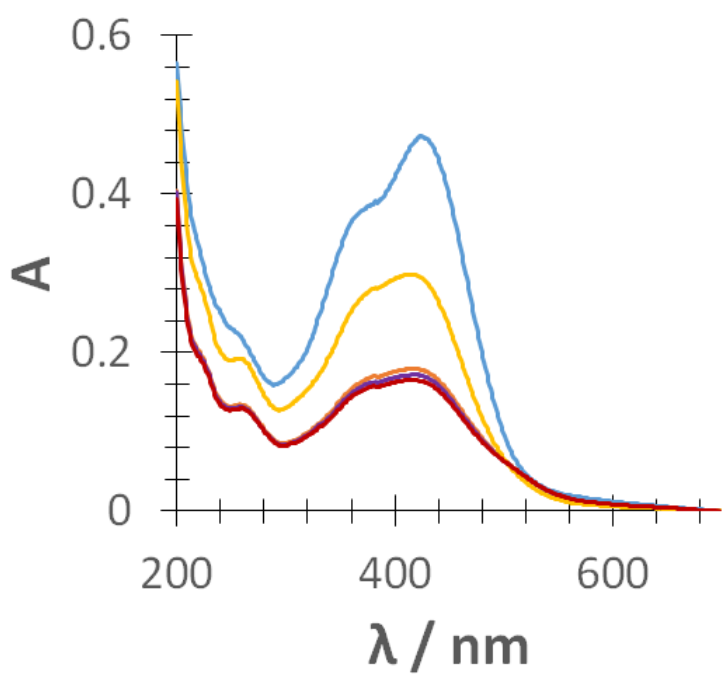

b)

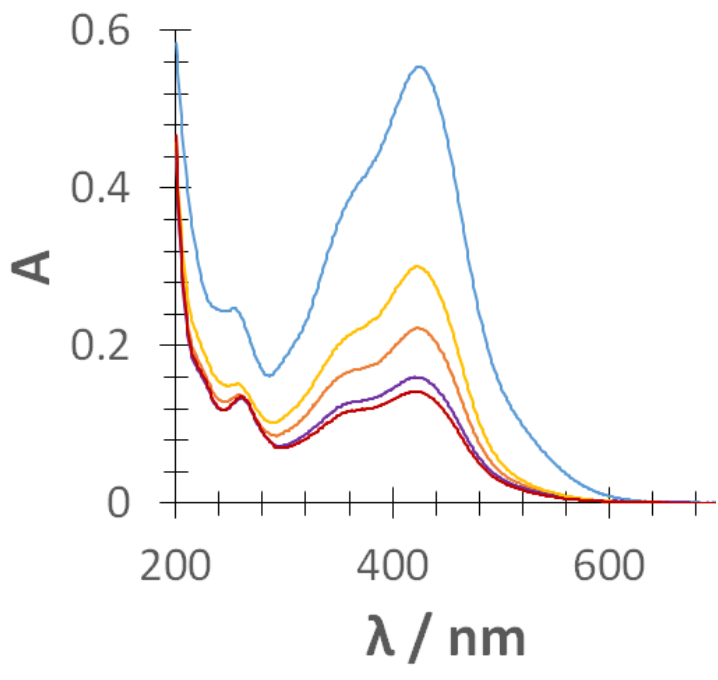




\section{c)}

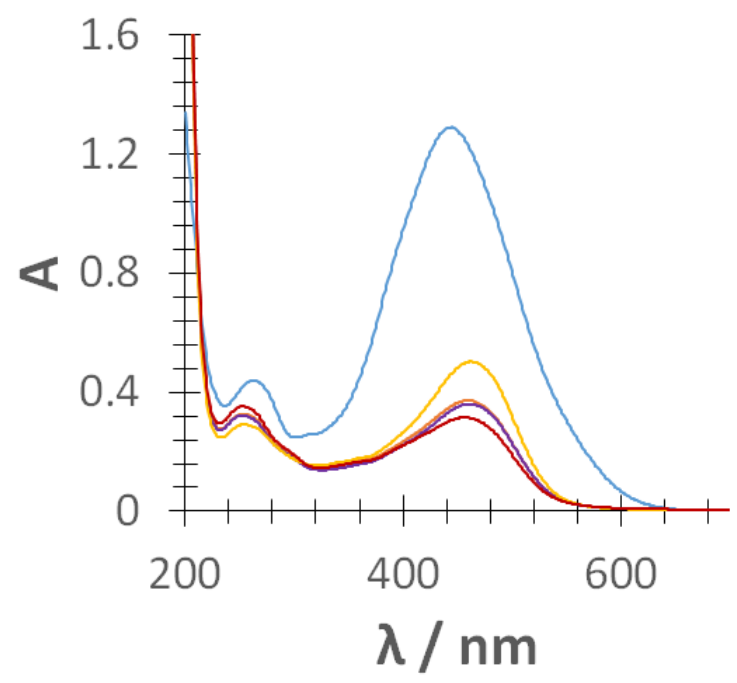

Figura 2. Espectros de absorción de una disolución de curcumina [32.5 $\mu \mathrm{M}]$ en medio acuoso bajo diferentes condiciones experimentales. a) pH $3.576 \pm 0.001$, b) $7.025 \pm 0.001$ y c) $\mathrm{pH} 10.526 \pm 0.001$. Sin control de ninguna variable experimental $(-)$, ausencia de luz (-), burbujeo de $\mathrm{N}_{2(g)}(-)$, temperatura constante $(21.0 \pm 0.1)^{\circ} \mathrm{C}(-)$ y control de todos las variables al mismo tiempo (-).

En general, se ve que al considerar un control de todas las variables experimentales al mismo tiempo el espectro de absorción muestra una mejor definición en los tres casos de $\mathrm{pH}$ estudiados aunque es de destacar que a pH 3.576 el efecto es más marcado. De manera particular a pH $3.576 \pm 0.001$ el espectro de absorción registrado cuando se trabaja en ausencia de luz así como el registrado cuando se trabaja en atmósfera inerte de $\mathrm{N}_{2(g)}$ no presenta diferencia alguna con aquel registrado cuando no existe control experimental. Solo se logra distinguir una banda a $263 \mathrm{~nm}$ y una banda que se extiende desde 357 a $423 \mathrm{~nm}$. El espectro de absorción registrado a temperatura constante muestra prácticamente el mismo comportamiento espectral discutido previamente pero con la diferencia que empieza a distinguirse el máximo de absorción a $423 \mathrm{~nm}$. La banda a $263 \mathrm{~nm}$ presenta un incremento en el valor de absorbancia del $30 \%$ y un $44 \%$ para el máximo de absorción a $423 \mathrm{~nm}$. Cuando se controlan todas las variables al mismo tiempo no solo se define mejor el máximo de absorción a $423 \mathrm{~nm}$ sino que la banda presenta un incremento del $65 \%$ con respecto al espectro registrado en ausencia de control experimental. En el caso de la banda a $263 \mathrm{~nm}$ el incremento es del $40 \%$.

A pH 7.025 se aprecia una banda a 263 nm y un máximo de absorción a 423 $\mathrm{nm}$. Se aprecia que al ir evaluando cada variable experimental sobre el sistema de estudio la absorbancia de la longitud de onda máxima también va en incremento: un $12 \%$ cuando se trabaja en atmósfera inerte de $\mathrm{N}_{2(\mathrm{~g})}$, un $36 \%$ cuando hay ausencia de luz, un $53 \%$ cuando se mantiene temperatura constante y un $74 \%$ cuando se mantienen todas las condiciones controladas al mismo tiempo. Todos los porcentajes 
anteriores son calculados con respecto al espectro que no tiene ningún control experimental. En el caso de la banda a $263 \mathrm{~nm}$, esta no presenta cambios entre cada variable estudiada. Solo cuando se trabaja bajo el control de todas las variables experimentales es cuando el valor de absorbancia se incrementa hasta un $41 \%$.

Para el caso a pH 10.526 se aprecia una banda a $254 \mathrm{~nm}$ y un máximo de absorción a $468 \mathrm{~nm}$. Se aprecia que al ir evaluando cada variable experimental sobre el sistema de estudio la absorbancia de la longitud de onda máxima también va en incremento: un $7 \%$ cuando se trabaja en atmósfera inerte de $\mathrm{N}_{2(\mathrm{~g})}$, un $11 \%$ cuando hay ausencia de luz, y un $30 \%$ cuando se mantiene temperatura constante. Sin embargo, cuando en el sistema se controlan todas las variables experimentales al mismo tiempo, se incrementa el valor de absorbancia del máximo de absorción hasta un $77 \%$ con un corrimiento hacia longitudes de onda menor de hasta $36 \mathrm{~nm}$. Todos los porcentajes anteriores son calculados con respecto al espectro que no tiene ningún control experimental. En el caso de la banda a $254 \mathrm{~nm}$, esta no presenta cambios entre cada variable estudiada; solo cuando se trabaja bajo el control de todas las variables experimentales es cuando la banda a $254 \mathrm{~nm}$ se recorre a $263 \mathrm{~nm}$. El incremento en absorbancia de esta banda es del $20 \%$ con respecto a si no hay control experimental.

De esta manera queda demostrado que la respuesta de absorbancia de la curcumina en medio acuoso para los tres casos de $\mathrm{pH}$ estudiados se ve mejorada cuando al mismo tiempo se trabaja en ausencia de luz, con previo burbujeo y manteniendo una atmósfera con gas $\mathrm{N}_{2(\mathrm{~g})}$ así como estableciendo una temperatura constante de $(21.0 \pm 0.1)^{\circ} \mathrm{C}$.

\subsection{Estudio de la estabilidad de la curcumina en medio acuoso. Cálculo de la velocidad de degradación $\left(V_{\mathrm{deg}}\right)$.}

Para estudiar cuál es el impacto de trabajar sin ningún control experimental y bajo condiciones controladas de: ausencia de luz, con previo burbujeo y manteniendo una atmósfera con gas $\mathrm{N}_{2}(\mathrm{~g})$ así como estableciendo una temperatura constante de $(21.0 \pm 0.1)^{\circ} \mathrm{C}$ sobre la velocidad de degradación de la curcumina en medio acuoso, se monitorea por espectrofotometría de UV-Vis y por voltamperometría cíclica la respuesta como una función del tiempo. 


\subsubsection{Reactivos.}

Curcumina grado analítico marca Sigma Aldrich ( $\geq 99.5 \%)$ sin ningún tratamiento de purificación posterior, lentejas de $\mathrm{NaOH}$ marca Macron, $\mathrm{HClO}_{4}$

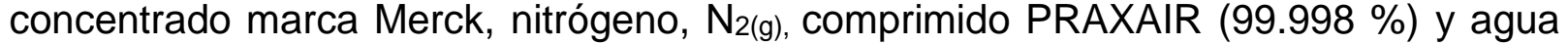
desionizada $(18.2 \mathrm{M} \Omega$ ) libre de materia orgánica obtenida con un filtro PURE-LAB Plus UV.

\subsubsection{Equipo.}

La lectura de $\mathrm{pH}$ se realiza con un potenciómetro marca sensION equipado con un electrodo de vidrio de $\mathrm{Ag}^{\circ} \mid \mathrm{AgCl}[\mathrm{KCl}, 3 \mathrm{M}]$ marca Hach $5014 \mathrm{~T}$.

Para el control de la temperatura se utiliza un recirculador Grant W14.

Cada espectro de absorción se registra mediante un espectrofotómetro Lambda 20 UV-Vis equipado con celdas de cuarzo $1 \mathrm{~cm}$ de longitud de paso óptico. El intervalo de adquisición de los espectros es de 200 a 700 nm a una velocidad de 1960 $\mathrm{nm} \mathrm{min}^{-1}$.

Para los registros voltamperométricos se utiliza una celda convencional con capacidad para tres electrodos siendo el electrodo de pasta de carbono el electrodo de trabajo preparado de acuerdo al método de Ramírez-Silva [28, 29]. Se utiliza un electrodo de referencia de $\mathrm{Ag}^{\circ} \mid \mathrm{AgCl}[\mathrm{KCl}, 3 \mathrm{M}]$ marca BAS MF-2052 y un alambre de platino BAS MW-1032 como contraelectrodo. Los registros voltamperométricos se hacen utilizando una ventana de potencial de $-350 \mathrm{mV}$ a $1000 \mathrm{mV}$, a una velocidad de barrido de $100 \mathrm{mV} \mathrm{s}^{-1}$ y considerando un ciclo de barrido. Entre cada barrido de potencial se renovó la superficie del electrodo mediante el pulimiento sobre papel.

\subsubsection{Método.}

Monitoreo de la estabilidad de la curcumina por espectrofotometría de UV-Vis. Se monitorea la respuesta de absorbancia de una disolución de trabajo de curcumina [32.5 $\mu \mathrm{M}]$ proveniente de una solución stock $[0.002 \mathrm{M}]$ la cual es previamente preparada en $\mathrm{NaOH}$ [5 mM] (esta se mantiene en refrigeración, ver Apéndice 1) a tres diferentes valores de $\mathrm{pH}: 3.576 \pm 0.001,7.025 \pm 0.001$ y $10.526 \pm 0.001$. Estos registros se hacen de manera independiente y bajo dos diferentes condiciones:

a) Sin control. No se impone cuidado alguno sobre las disoluciones de trabajo.

b) Control experimental. El cual consiste en trabajar en ausencia de luz, burbujear previamente por 5 minutos la disolución de trabajo con $\mathrm{N}_{2}(\mathrm{~g})$ manteniendo posteriormente una atmósfera inerte con este mismo gas e imponiendo una 
temperatura constante de $(21.0 \pm 0.1){ }^{\circ} \mathrm{C}$ durante el tiempo que dure el experimento.

Monitoreo de la estabilidad de la curcumina por voltamperometría cíclica. Se obtiene la respuesta de corriente de una disolución de trabajo de curcumina [32.5 $\mu \mathrm{M}]$ proveniente de una solución stock [0.002 M] la cual es preparada en $\mathrm{NaOH}$ [5 mM] (esta se mantiene en refrigeración, ver Apéndice 1) a tres diferentes valores de $\mathrm{pH}$ : $3.576 \pm 0.001,7.025 \pm 0.001$ y $10.526 \pm 0.001$. Estos registros se hacen de manera independiente y bajo diferentes condiciones experimentales, las cuales han sido mencionadas en los puntos a y $b$ del párrafo anterior.

Es importante destacar que para mejorar la respuesta a tiempo cero cuando no hay control experimental y cuando se trabaja bajo condiciones controladas, la preparación de la disolución de curcumina de trabajo fue lo último que se realizó mejorando así la respuesta a tiempo cero, lo cual se puede observar al comparar los espectros de absorción sin control experimental de la Figura 2 y de la Figura 3.

\subsubsection{Monitoreo de la estabilidad de la curcumina por espectrofotometría de UV-} Vis.

En la Figura 3 se muestran una familia de espectros de absorción a diferentes valores de $\mathrm{pH}$ y diferentes condiciones experimentales. De manera general a $\mathrm{pH}$ $3.576 \pm 0.001$ y pH $7.025 \pm 0.001$ se muestran dos transiciones electrónicas en común: el máximo de absorción localizado a $423 \mathrm{~nm}(432 \mathrm{~nm}$ para pH $10.526 \pm 0.001)$ y otra banda a $263 \mathrm{~nm}$. De acuerdo a lo reportado por Balasubramanian [22], la transición a $423 \mathrm{~nm}(432 \mathrm{~nm}$ para $\mathrm{pH} 10.526 \pm 0.001)$ correspondería a una transición de tipo $n$ a $\pi^{*}$ característica de compuestos donde están presentes pares de electrones libres asociados con heteroátomos. Acorde a la Figura 1 serían los oxígenos del grupo ceto enol a los que se le asociaría esta transición. En el caso de la banda a $263 \mathrm{~nm}$, Balarubramanian [22] reporta que es una transición electrónica de tipo $\pi$ a $\pi^{*}$ característica de compuestos con dobles enlaces conjugados. De acuerdo a la estructura de la curcumina, serían los grupos fenólicos de ambos extremos de la molécula los responsables de dicha transición. La banda a $355 \mathrm{~nm}$ que se observa a $\mathrm{pH} 3.576 \pm 0.001$ y pH $7.025 \pm 0.001$ es una transición que de acuerdo a Balasubramanian [22] corresponde a una transición del tipo $\pi$ a $\pi^{*}$ asociada a los dobles enlaces de la cadena alifática

Así mismo, en la Figura 3 se muestra como la absorbancia cae drásticamente como una función del tiempo independientemente si las condiciones experimentales son controladas o no, ver inserto en Figuras 3 a y $3 \mathrm{~b}$ para el caso a pH $3.576 \pm 0.001$. Las Figuras $3 \mathrm{c}$ y $3 \mathrm{~d}$ son los espectros de absorción a pH $7.025 \pm 0.001$. A este valor de $\mathrm{pH}$ también se muestra una variación temporal de la absorbancia, ver insertos. Es importante mencionar que el comportamiento en la Figura $3 \mathrm{c}$ es muy parecido al observado en medio ácido bajo condiciones experimentales no controladas. Sin 
embargo, a pH $10.526 \pm 0.001$ el cambio temporal de la absorbancia no es tan drástico con respecto a $\mathrm{pH}$ ácido o neutro independientemente si se controlan o no las condiciones experimentales, ver insertos de las Figuras $3 e$ y $3 f$.

A partir de estos resultados de la variación de la absorbancia se puede definir el porcentaje de degradación de la curcumina (\% DC) en disolución, ver Ecuación 1:

$$
\% D C=100 \times\left[\left(A_{o}-A_{t}\right) /\left(A_{\circ}\right)\right]
$$

\section{Ecuación 1}

Donde $A_{o}$ es la absorbancia inicial a tiempo cero, y $A_{t}$ es la absorbancia al tiempo $t$ en la longitud de onda máxima.
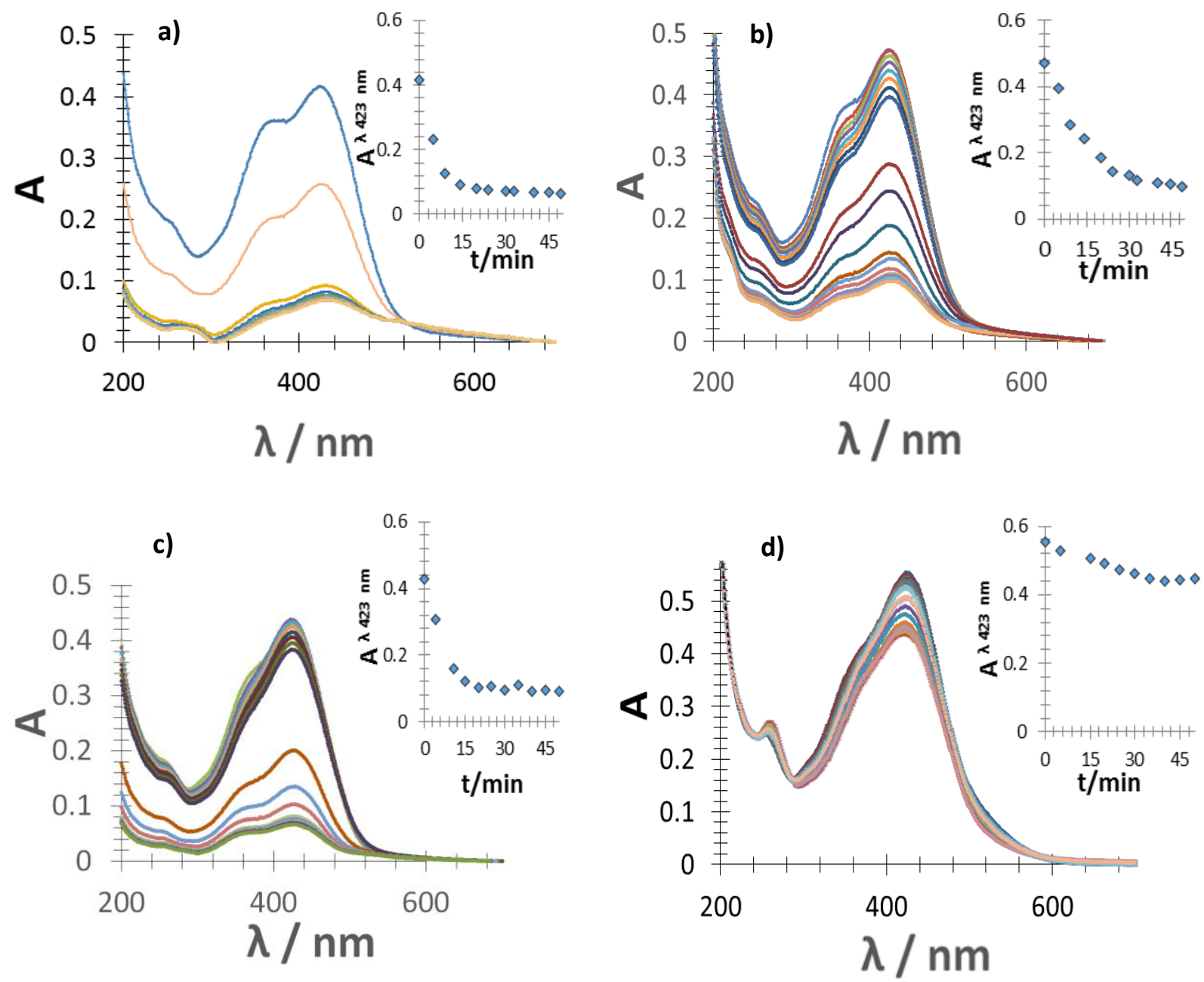

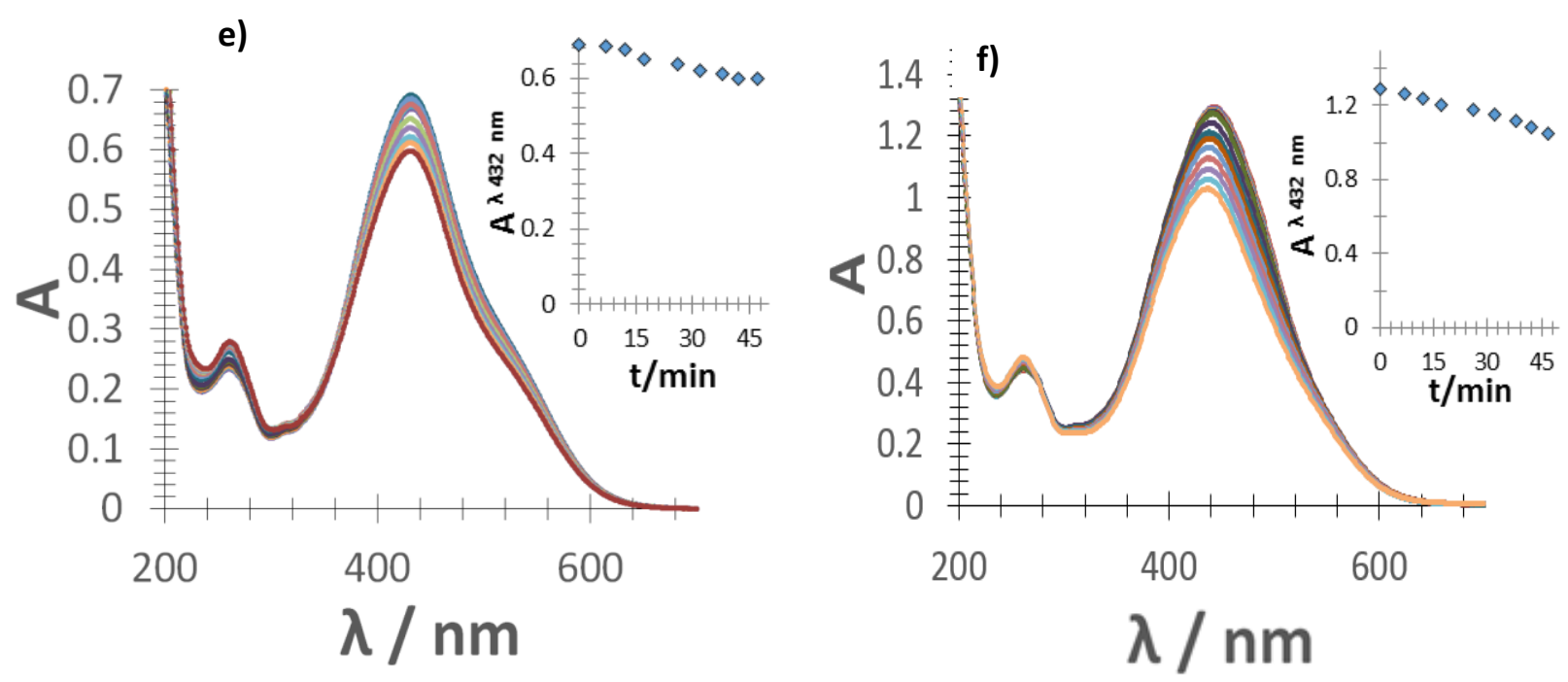

Figura 3. Familia de espectros de absorción de una disolución acuosa de curcumina [32.5 $\mu \mathrm{M}]$ a diferentes valores de $\mathrm{pH}$. a) y b) $\mathrm{pH} 3.576 \pm 0.001$; c) y d) $\mathrm{pH} 7.025 \pm 0.001$; e) y f) $10.526 \pm 0.001$. Izquierda: Sin control de las condiciones experimentales. Derecha: Control de las condiciones experimentales. Los insertos muestran la variación de la absorbancia a $423 \mathrm{~nm}$ (a-d) y $432 \mathrm{~nm}$ (e y f).

En la Figura 4 se muestra la variación del \% DC como una función del tiempo para diferentes valores de $\mathrm{pH}$ y condiciones experimentales. En los primeros 7 minutos el \% DC (cuando no hay control del sistema) es cercano al $45 \%$ y $30 \%$ para $\mathrm{pH} 3.576 \pm 0.001$ y pH $7.025 \pm 0.001$ respectivamente mientras que a pH $10.526 \pm$ 0.001 el \% DC es apenas del $2 \%$. Sin embargo, al mantener el control de las condiciones experimentales durante el tiempo que dura el experimento, el \% DC es del $16 \%$ y $5 \%$ para pH $3.576 \pm 0.001$ y pH $7.025 \pm 0.001$ respectivamente. En el caso de $\mathrm{pH}$ básico el \% DC no supera el $1 \%$. En todos los casos analizados la curcumina en disolución es más estable conforme el pH aumenta lo cual es contrario a lo reportado por Tonnesen y Karlsen $[16,17]$ y Wang et al. [18].
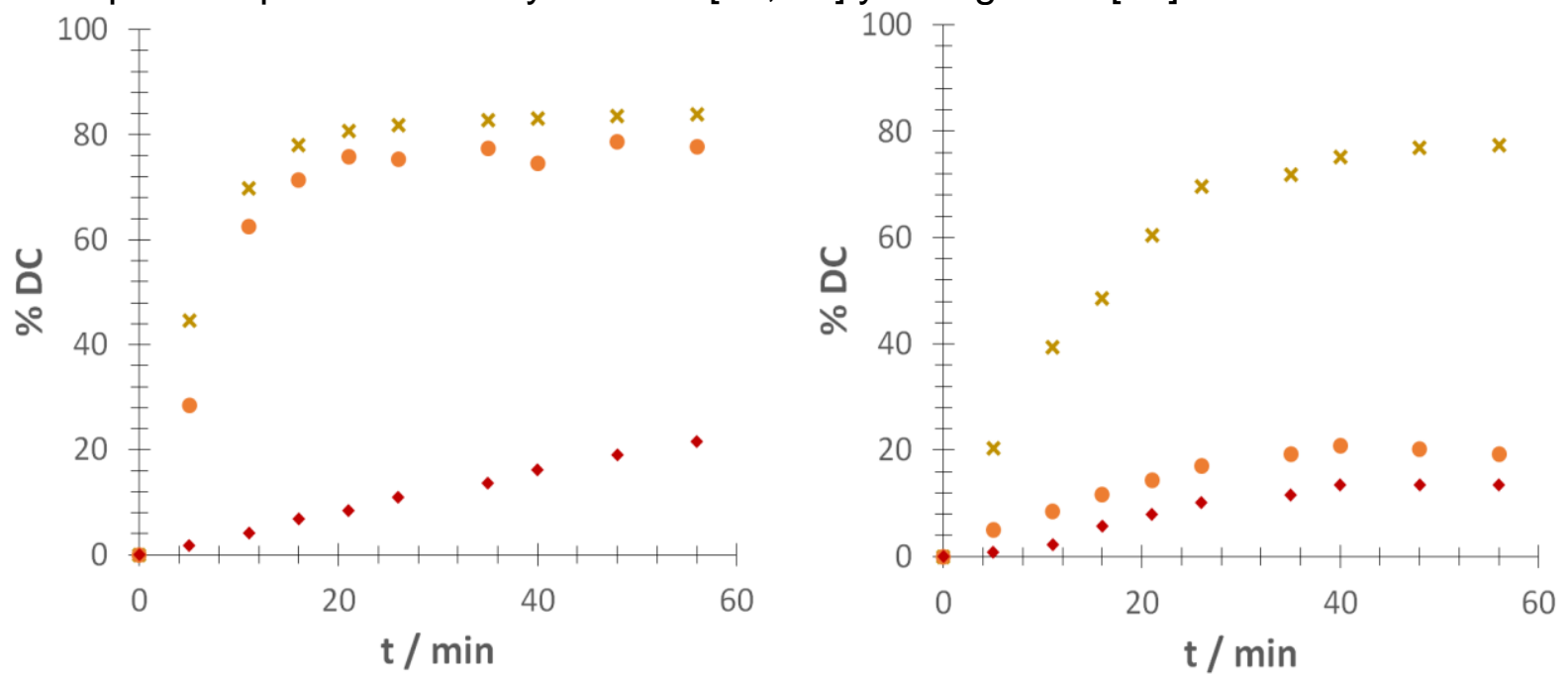

Figura 4. Porcentaje de degradación de la curcumina $[32.5 \mu \mathrm{M}]$ en disolución acuosa $(\% \mathrm{DC})$ como una función del tiempo a diferentes valores de pH: (X) $3.576 \pm 0.001,(\bullet) 7.025 \pm 0.001$ y (४) $10.526 \pm$ 0.001 . (Izquierda) condiciones no controladas, (Derecha) condiciones controladas. 
A partir de los datos mostrados en la Figura 4 se puede definir la velocidad de degradación, $V_{\text {deg, }}$ como la derivada del porcentaje de degradación de la curcumina en disolución acuosa, \% DC, con respecto al tiempo, ver Ecuación 2:

$$
\mathrm{V}_{\text {deg }}=\mathrm{d}(\% \mathrm{DC}) / \mathrm{dt}
$$

Ecuación 2

La variación temporal del \% DC es prácticamente lineal durante los primeros 20 minutos, por lo tanto, la $V_{\text {deg, }}$ puede estimarse a partir de la pendiente de estas líneas rectas, ver Tabla 1.

Tabla 1. Valores de velocidad de degradación $\left(\mathrm{V}_{\text {deg }}\right)$ de una disolución acuosa de curcumina a diferentes valores de $\mathrm{pH}$ y condiciones experimentales.

\begin{tabular}{|ccc|}
\hline & \multicolumn{2}{c|}{$\mathrm{V}_{\mathrm{deg}} / \% \mathrm{DC} \mathrm{min}^{-1}$} \\
$\mathbf{p H}$ & Condiciones No controladas & Condiciones controladas \\
\hline $\mathbf{3 . 5 7 6} \pm \mathbf{0 . 0 0 1}$ & $7.80 \pm 0.75$ & $2.86 \pm 0.29$ \\
\hline $\mathbf{7 . 0 2 5} \pm \mathbf{0 . 0 0 1}$ & $4.78 \pm 0.59$ & $0.47 \pm 0.03$ \\
\hline $\mathbf{1 0 . 5 2 6} \pm \mathbf{0 . 0 0 1}$ & $0.39 \pm 0.02$ & $0.34 \pm 0.02$ \\
\hline
\end{tabular}

De los valores mostrados en la Tabla 1 , se puede notar que la $V_{\text {deg }}$ es mayor conforme el $\mathrm{pH}$ se vuelve más ácido, por tanto, la estabilidad de la curcumina es mayor conforme el $\mathrm{pH}$ se vuelve más básico. Para cada valor de $\mathrm{pH}$, la $\mathrm{V}_{\text {deg }}$ disminuye siempre y cuando se mantenga un control de las condiciones experimentales.

\subsubsection{Monitoreo de la estabilidad de la curcumina por voltamperometría cíclica.}

Dado que la curcumina tiene grupos fácilmente oxidables $(-\mathrm{OH})$, se esperaría que algún cambio en su estructura a causa de una inestabilidad química (oxidación) pueda ser evidenciada por voltamperometría cíclica toda vez que es una técnica instrumental que permite obtener información del proceso de oxidación del sistema bajo estudio.

En la Figura 5 se muestran los voltamperogramas cíclicos de la curcumina [32.5 $\mu \mathrm{M}$ ] registrados cada 5 minutos en presencia de iones percloratos [0.068 M]. El monitoreo de la respuesta fue sin control experimental (izquierda) y bajo condiciones controladas (derecha) a tres valores de $\mathrm{pH}$ : a) y b) $\mathrm{pH} 3.576 \pm 0.001$; c) y d) $\mathrm{pH} 7.025$ \pm 0.001 ; e y f) $\mathrm{pH} 10.526 \pm 0.001$. Conforme se incrementa el valor de $\mathrm{pH}$, los procesos de oxidación se vuelven más complicados de distinguir y de interpretar razón por la cual se toma el valor de potencial de pico anódico, Ep ${ }^{\mathrm{a}}$, igual a $0.670 \mathrm{~V}$ como referencia. De esta manera en los insertos de cada figura se muestra la caída del valor de intensidad de corriente a $0.670 \mathrm{~V}$. 
a)

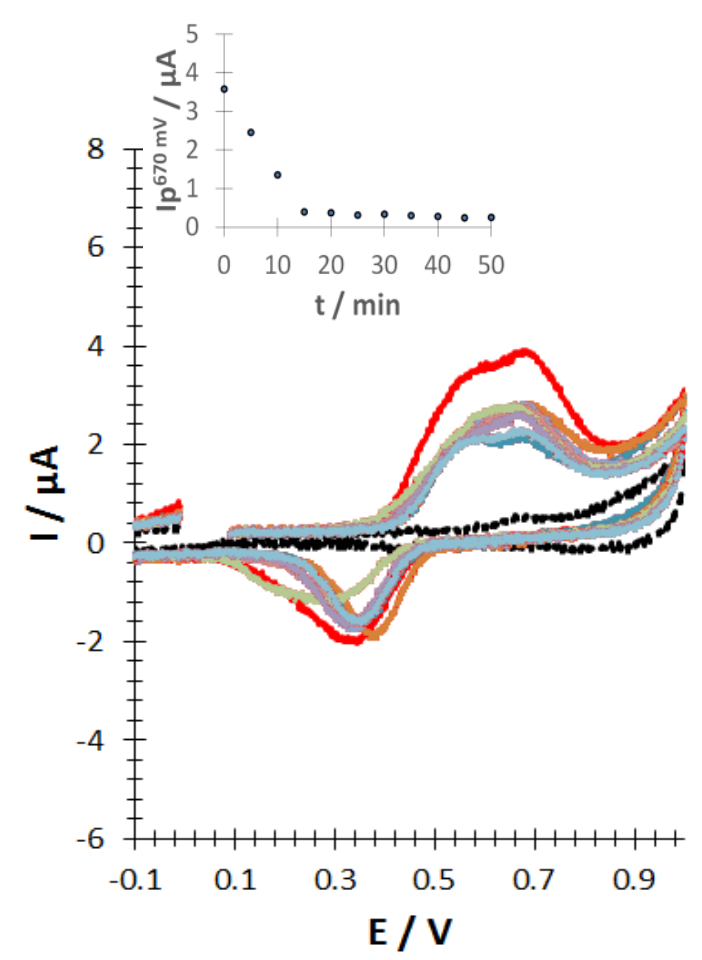

c)

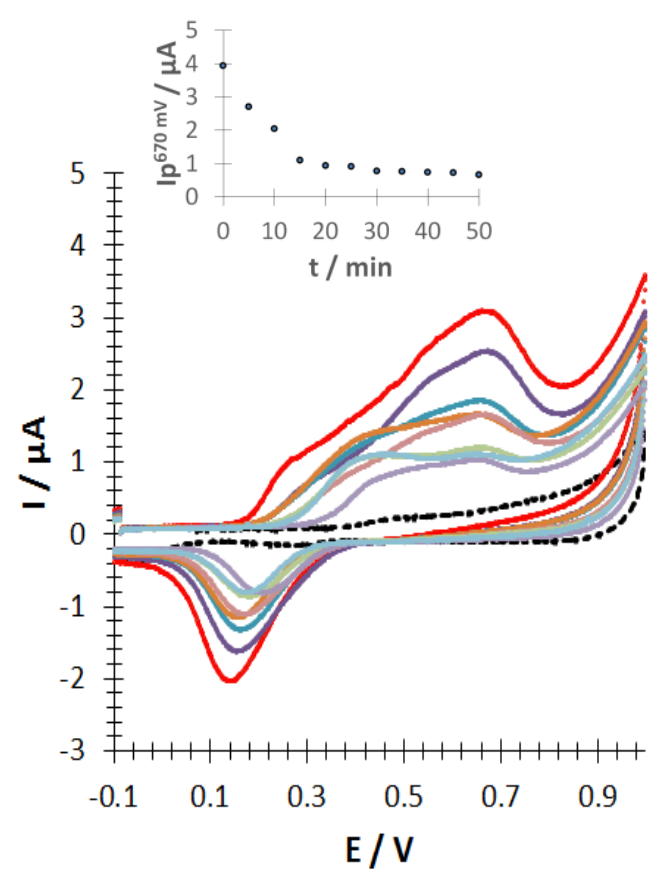

b)

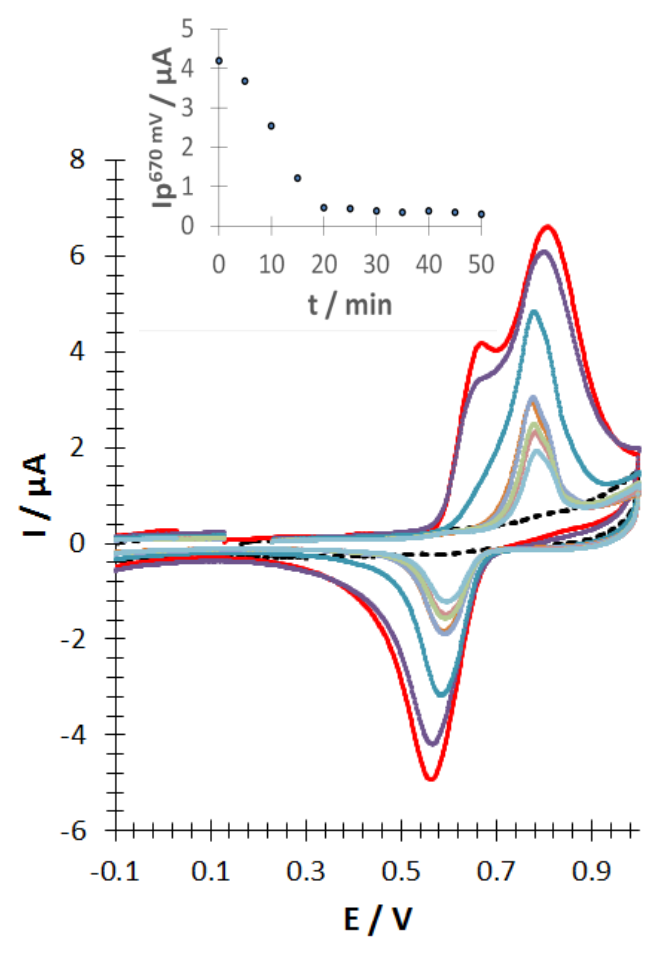

d)

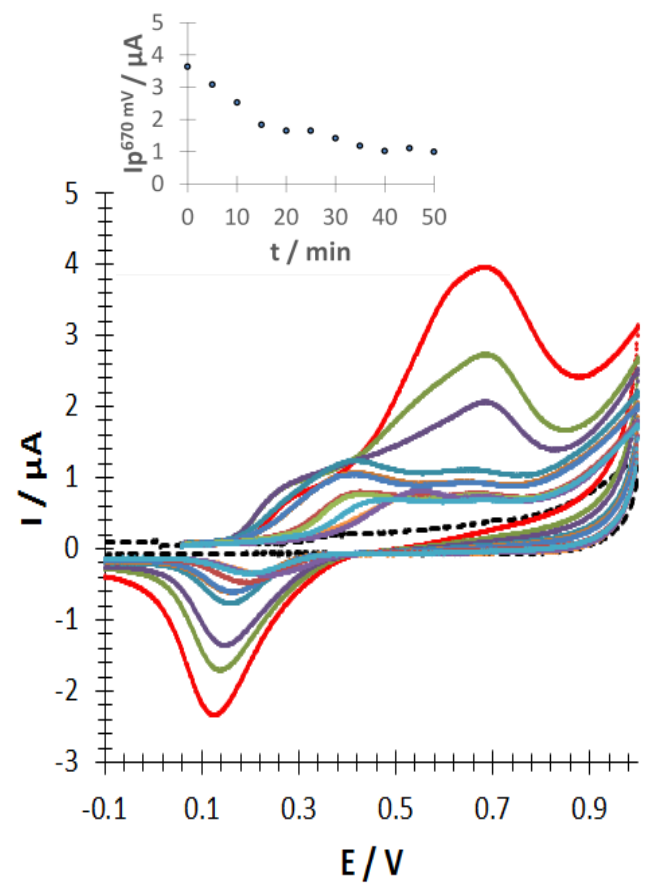


e)

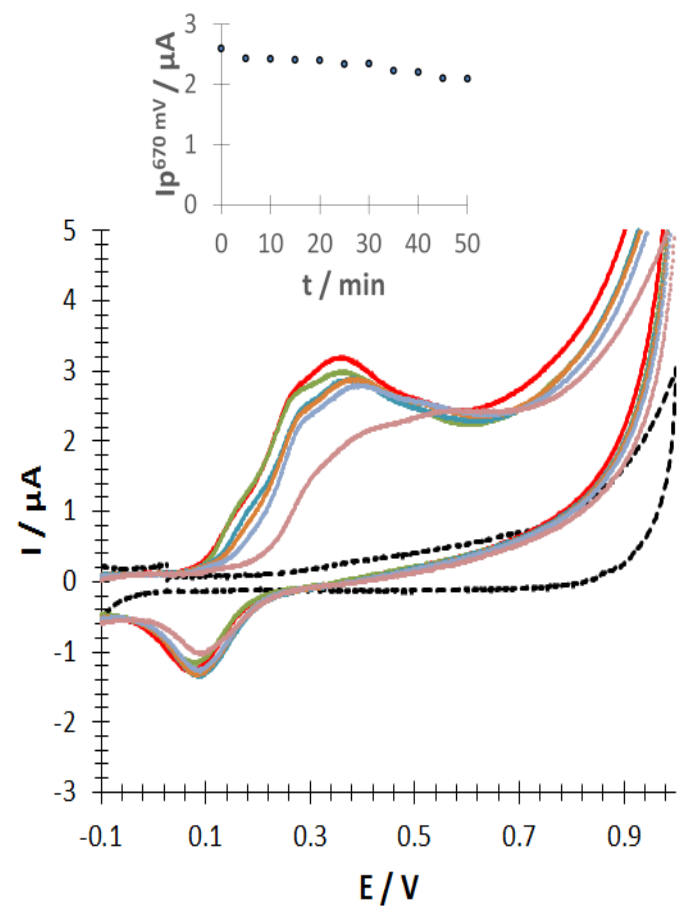

f)

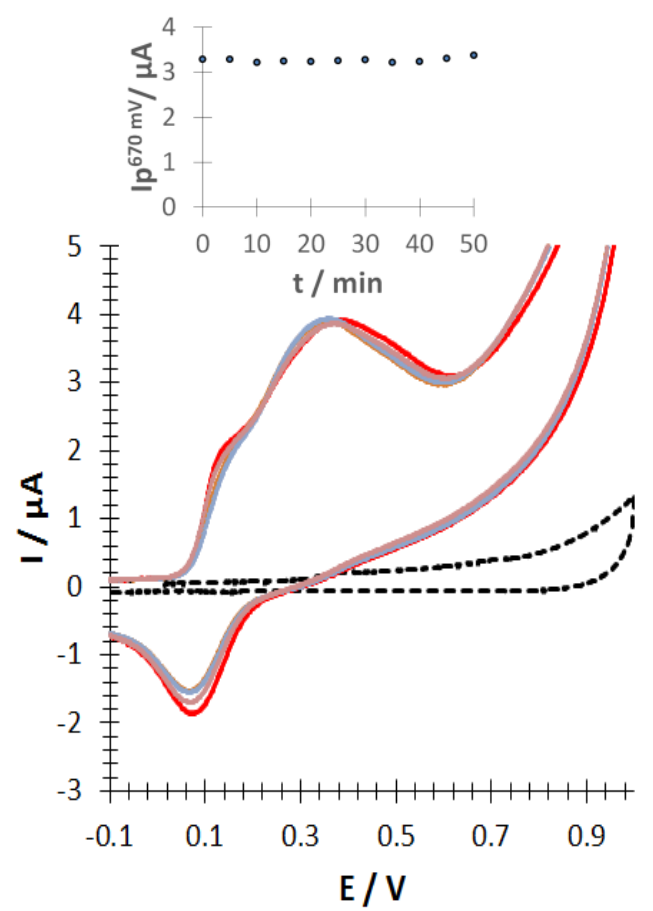

Figura 5. Voltamperogramas cíclicos de una disolución acuosa de curcumina [32.5 $\mu \mathrm{M}]$ registrados cada 5 minutos en presencia de iones percloratos $[0.068 \mathrm{M}]$, bajo condiciones no controladas (izquierda) y controladas (derecha) a tres diferentes valores de $\mathrm{pH}$. a) y b) $\mathrm{pH} 3.576 \pm 0.001 \mathrm{c}$ ) y d) $\mathrm{pH}$ $7.025 \pm 0.001$ y e) y f) $\mathrm{pH} 10.526 \pm 0.001$. El registro punteado es el blanco. Los insertos muestran la caída de corriente de pico a $670 \mathrm{mV}$ como una función del tiempo.

A pH $3.576 \pm 0.001$ existe un primer proceso de oxidación localizado a un valor de potencial de pico anódico, Ep ${ }^{a}$ igual a $0.670 \mathrm{~V}$ el cual solo se define muy bien bajo condiciones controladas y que va perdiendo su forma con el paso del tiempo hasta desaparecer por completo después de 15 minutos. Existe un segundo proceso de oxidación con valor de potencial de pico anódico, Ep॥ ${ }^{\mathrm{a}}$ igual a $0.813 \mathrm{~V}$ el cual después de 60 minutos se desplaza a Ep $\|^{a}{ }^{\prime} 0.781 \mathrm{~V}$ con una intensidad de corriente cerca del $70 \%$ menos del valor inicial. Después del potencial de inversión y conforme se llega a valores de potencial más negativos se distingue un proceso de reducción, $\mathrm{Ep}_{\mathrm{I}}{ }^{\mathrm{C}}$ igual a $0.570 \mathrm{~V}$ cuyo valor de corriente también disminuye con el paso del tiempo. Mediante un estudio $E_{\lambda}$ queda demostrado que el pico de reducción a $0.570 \mathrm{~V}$ corresponde al producto de oxidación del pico anódico localizado a $0.670 \mathrm{~V}$ (ver Apéndice 2 para más detalles de la propuesta de mecanismo de oxidación).

A pH $7.025 \pm 0.001$, el potencial del primer proceso de oxidación no está bien definido; sin embargo se puede observar que la respuesta va perdiendo su forma menos rápida con respecto a pH $3.576 \pm 0.001$. Esto quiere decir que la solución de curcumina a pH 7.025 \pm 0.001 es más estable que a pH $3.576 \pm 0.001$.

A diferencia de los casos anteriores, a pH $10.526 \pm 0.001$ la señal no mostró un cambio significativo en la respuesta de corriente durante $60 \mathrm{~min}$ (bajo condiciones controladas) sin embargo, al igual que a $\mathrm{pH} 7.025 \pm 0.001$ los procesos no se definen 
claramente. Una posible causa de esto es que los grupos susceptibles de oxidación son semejantes en ambos extremos de la molécula y por tanto, la respuesta es la suma de ambos procesos.

A partir de estos resultados de la variación de la corriente en función del tiempo se puede definir el porcentaje de degradación de la curcumina (\% DC) en disolución (Figura 6), ver Ecuación 3:

$$
\% D C=100 \times\left[\left(I p_{\circ} 670 \mathrm{mV}-I p^{670 \mathrm{mV}}\right) /\left(\mid \mathrm{p}_{\circ} 670 \mathrm{mV}\right)\right]
$$

Ecuación 3

Donde $1 \mathrm{p}_{o}^{670 \mathrm{mV}}$ es la corriente inicial a tiempo cero y $1 \mathrm{p}^{670 \mathrm{mV}}$ es la corriente al tiempo $t$.
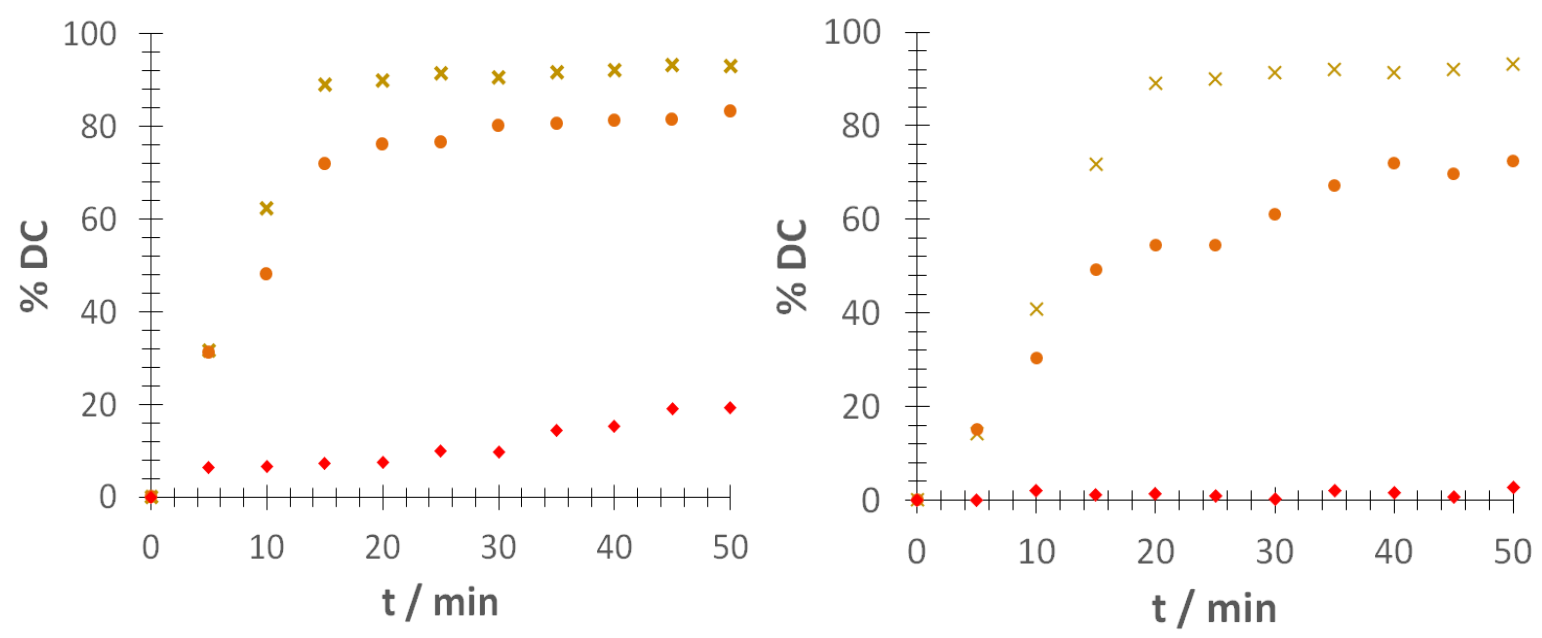

Figura 6. Porcentaje de degradación de la curcumina [32.5 $\mu \mathrm{M}]$ en disolución acuosa (\% DC) como una función del tiempo a diferentes valores de $\mathrm{pH}$ : (X) $3.576 \pm 0.001$, (•) $7.025 \pm 0.001$ y (\$) $10.526 \pm 0.001$. (Izquierda) condiciones no controladas, (Derecha) condiciones controladas.

Al igual que en el análisis por espectrofotometría de UV-Vis, la variación temporal del \% DC (Figura 6) es prácticamente lineal durante los primeros 20 minutos, por lo tanto, la $V_{\text {deg, }}$ puede estimarse a partir de la pendiente de estas líneas rectas, ver Tabla 2. 
Tabla 2. Valores de velocidad de degradación $\left(V_{\text {deg }}\right)$ de una disolución acuosa de curcumina a diferentes valores de $\mathrm{pH}$ y condiciones experimentales.

\begin{tabular}{|ccc|}
\hline & \multicolumn{2}{c|}{$\mathrm{V}_{\mathrm{deg}} / \% \mathrm{DC} \mathrm{min}^{-1}$} \\
& Condiciones No controladas & Condiciones controladas \\
\hline $\mathbf{3 . 5 7 6} \pm \mathbf{0 . 0 0 1}$ & $5.96 \pm 0.17$ & $4.85 \pm 0.54$ \\
\hline $\mathbf{7 . 0 2 5} \pm \mathbf{0 . 0 0 1}$ & $4.66 \pm 0.38$ & $3.27 \pm 0.13$ \\
\hline $\mathbf{1 0 . 5 2 6} \pm \mathbf{0 . 0 0 1}$ & $0.44 \pm 0.21$ & $0.11 \pm 0.08$ \\
\hline
\end{tabular}

A partir de los valores mostrados en la Tabla 2, se puede demostrar que la

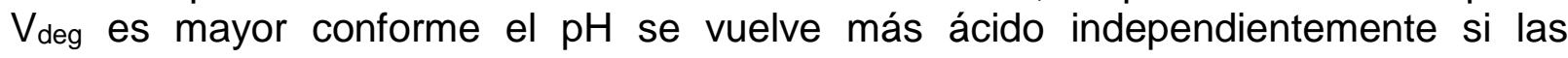
condiciones del medio son controladas o no. De esta manera se corrobora lo analizado por espectrofotometría de UV-Vis, la estabilidad de la curcumina es mayor conforme el $\mathrm{pH}$ se vuelve más básico. Para cada valor de $\mathrm{pH}$, la $\mathrm{V}_{\text {deg }}$ disminuye siempre y cuando se mantenga un control de las condiciones experimentales.

\subsection{Conclusiones.}

En el presente capítulo queda demostrado a tres valores de $\mathrm{pH} 3.576,7.025$ y 10.526 como influyen las variables experimentales (presencia de luz, $\mathrm{O}_{2(\mathrm{~g})}$ y temperatura) en la estabilidad química de la curcumina en disolución acuosa y por tanto en su respuesta espectrofotométrica y voltamperométrica como una función del tiempo. Se calculan los valores de $\mathrm{V}_{\text {deg }}$ a cada valor de $\mathrm{pH}$ encontrando que esta disminuye conforme el valor de pH es más básico. 


\section{CAPÍTULO 2. Determinación de las constantes de acidez de la curcumina por espectrofotometría de UV-Vis.}

"El coraje de un gran líder para cumplir su visión proviene de la pasión, no de la posición" 


\subsection{Las constantes de acidez de la curcumina.}

De acuerdo a la estructura enólica de la curcumina, ver Figura 7, presenta al menos tres protones ácidos los cuales pueden estar asociados a tres constantes de acidez o pka's.

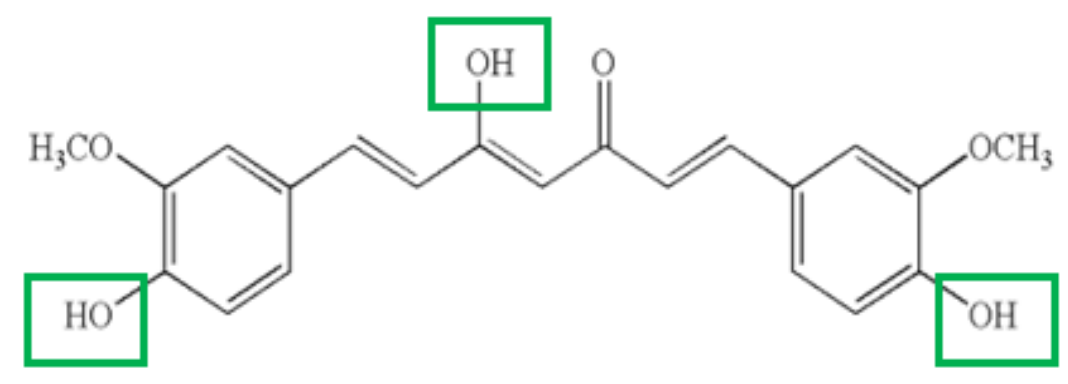

Figura 7. Estructura química de la curcumina en disolución acuosa, en cuadros verdes se encuentran encerrados los protones ácidos asociados a posibles valores de pKa.

Actualmente está reportado el cálculo de estas constantes de acidez, ver Tabla 3 , bajo diferentes métodos analíticos. Sin embargo, se puede apreciar la discrepancia entre los valores reportados. Esta discrepancia no solo radica en el reactivo de curcumina utilizado (si fue sintetizada u obtenida por alguna casa comercial), también influye si el reactivo fue purificado o no, el medio de reacción y la estrategia instrumental utilizada, o si existió algún tratamiento adicional sobre los datos obtenidos (software computacional). También es importante considerar si la disolución de trabajo utilizada fue la misma durante el tiempo que duró el experimento (variable del tiempo).

Considerando que en el Capítulo 1 de este trabajo se demuestra que la estabilidad de la curcumina es mayor conforme el pH se hace más básico contrario a lo reportado por Tonnesen y Karlsen [16, 17] y Wang et. al. [18] se considera imperante recalcular las constantes de acidez o valores de pKa bajo condiciones experimentales controladas (ya mencionadas en el Capítulo 1 de este trabajo) para garantizar así la confiabilidad de estos valores. 
Tabla 3. Valores de constantes de acidez reportados en la literatura para la curcumina en disolución acuosa.

\begin{tabular}{|c|c|c|c|c|}
\hline Método & $\begin{array}{c}\mathrm{pKa}_{1} \pm \boldsymbol{\sigma} \\
\text { Equilibrio: } \\
\mathrm{H}_{3} \mathrm{Cur}=\mathrm{H}_{2} \mathrm{Cur}^{-}+\mathrm{H}^{+}\end{array}$ & $\begin{array}{c}\mathrm{pKa}_{2} \pm \sigma \\
\text { Equilibrio: } \\
\mathrm{H}_{2} \mathrm{Cur}^{-}=\mathrm{HCur}^{2-}+\mathrm{H}^{+}\end{array}$ & $\begin{array}{c}\operatorname{pKa3}^{ \pm \sigma} \\
\text { Equilibrio: } \\
\text { HCur }^{2-}=\text { Cur }^{3-}+\mathrm{H}^{+}\end{array}$ & Ref. \\
\hline $\begin{array}{l}\text { HPLC con detector de fluorescencia. } \\
\text { Fuerza iónica } 0.10 \text { a } 0.15 \mathrm{M} \text {. } \\
\text { Temperatura } \\
(31.5 \pm 0.5)^{\circ} \mathrm{C} \text {. } \\
\text { Sistemas buffer: } \mathrm{KCl} / \mathrm{HCl} \text {, fosfatos y } \\
\text { carbonatos. } \\
\text { Curcumina sintetizada según el } \\
\text { método de Pabón. } \\
\text { La determinación de pka's se hace a } \\
\text { partir de una curva de } \mathrm{K}_{\text {deg }} \text { en función } \\
\text { del pH. }\end{array}$ & $7.75-7.80 \pm$ N.R. & $8.55 \pm 0.05$ & $9.05 \pm 0.05$ & 16 \\
\hline $\begin{array}{l}\text { Titulación espectrofotométrica con } \\
\text { HCl. Control de luz, burbujeo de } \mathrm{N}_{2(\mathrm{~g})} \text {. } \\
\text { Curcumina R.A. Merck } \\
\qquad(\geq 99 \%) \text {. } \\
\text { Refinamiento de datos mediante el } \\
\text { software SQUAD. }\end{array}$ & $8.38 \pm 0.04$ & $9.88 \pm 0.02$ & $10.51 \pm 0.01$ & 20 \\
\hline $\begin{array}{l}\text { UV-Vis con refinamiento de datos } \\
\text { mediante el software Sigma Plot. } \\
\text { Fuerza iónica 0.1 M. Temperatura (20 } \\
\pm 1)^{\circ} \mathrm{C} . \\
\text { Curcumina R.A. } \\
(\geq 99 \%) .\end{array}$ & $8.10 \pm$ N.R. & $10.45 \pm$ N.R. & N. R. & 21 \\
\hline $\begin{array}{c}\text { UV Vis } \\
\text { Método no reportado. }\end{array}$ & $8.55 \pm 0.05$ & $10.41 \pm 0.05$ & N. R. & 30 \\
\hline 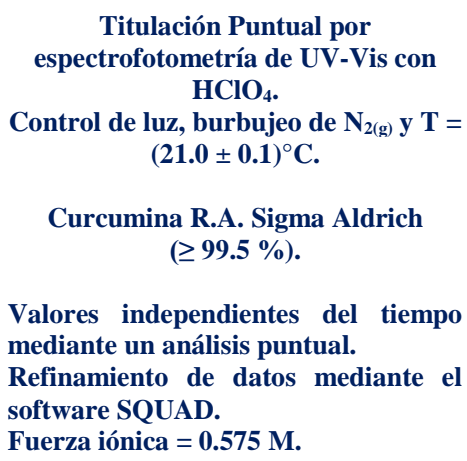 & $7.428 \pm 0.015$ & $9.552 \pm 0.024$ & $10.946 \pm 0.034$ & $\begin{array}{c}\text { Este } \\
\text { trabajo }\end{array}$ \\
\hline
\end{tabular}

\section{N.R. No Reportado}




\subsection{Determinación de las constantes de acidez de la curcumina bajo condiciones experimentales controladas en disolución acuosa.}

\subsubsection{Reactivos.}

Curcumina grado analítico marca Sigma Aldrich ( $\geq 99.5 \%$ ) sin ningún tratamiento adicional de purificación, lentejas de $\mathrm{NaOH}$ marca Macron, $\mathrm{HClO}_{4}$

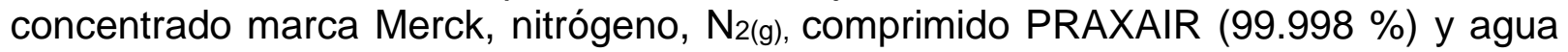
desionizada (18.2 $\mathrm{M} \Omega$ ) libre de materia orgánica obtenida con un filtro PURE-LAB Plus UV.

\subsubsection{Equipo.}

La lectura de $\mathrm{pH}$ se realiza con un potenciómetro marca sensION equipado con un electrodo de vidrio de $\mathrm{Ag}^{\circ} \mid \mathrm{AgCl}[\mathrm{KCl}, 3 \mathrm{M}]$ marca Hach $5014 \mathrm{~T}$.

Para el control de la temperatura se utiliza un recirculador Grant W14.

Cada espectro de absorción se registra mediante un espectrofotómetro Lambda 20 UV Vis equipado con celdas de cuarzo $1 \mathrm{~cm}$ de longitud de paso óptico. El intervalo de adquisición de los espectros es de 200 a 700 nm a una velocidad de 1960 $\mathrm{nm}$ min $^{-1}$.

\subsubsection{Método.}

Se preparan 32 disoluciones (medios de reacción) para la curcumina [32.5 $\mu \mathrm{M}$ ] y se ajustan a un $\mathrm{pH}$ determinado previo a la adición y lectura de una alícuota de curcumina proveniente de la disolución stock [0.002 M] previamente preparada en $\mathrm{NaOH}[5 \mathrm{mM}$ ] y que es mantenida en refrigeración. El pH se ajusta de $\mathrm{pH}$ básico a $\mathrm{pH}$ ácido. Es importante mencionar que durante el tiempo que dura el experimento, se trabaja bajo condiciones experimentales controladas las cuales se mencionan en el Capítulo 1 de este trabajo. El volumen $(5 \mathrm{~mL})$ y tiempo de agitación (previo a la lectura instrumental) fue el mismo para las 32 disoluciones. 
2.2.4 Cálculo de las constantes de acidez utilizando una estrategia gráfica y el programa computacional SQUAD.

En la Figura 8 se muestran la familia de espectros de absorción registrados por espectrofotometría de UV-Vis para la curcumina [32.5 $\mu \mathrm{M}$ ] en disolución acuosa. Se aprecia un efecto hipsocrómico, es decir, conforme el valor de $\mathrm{pH}$ disminuye, la banda principal a $475 \mathrm{~nm}$ se desplaza hacia valores de longitud de onda menores. Así mismo, se alcanza a distinguir la presencia de puntos isosbésticos los cuales están localizados a 306, 327, 382, 428 y $530 \mathrm{~nm}$. Estos puntos isosbésticos indican que dos o más especies en disolución presentan el mismo coeficiente de extinción molar a una misma longitud de onda determinada lo cual puede asociarse a que está presente uno o más equilibrios en disolución. Por la naturaleza del presente estudio, estos equilibrios serían de tipo ácido-base los cuales a su vez pueden asociarse a una 0 más constantes de acidez.

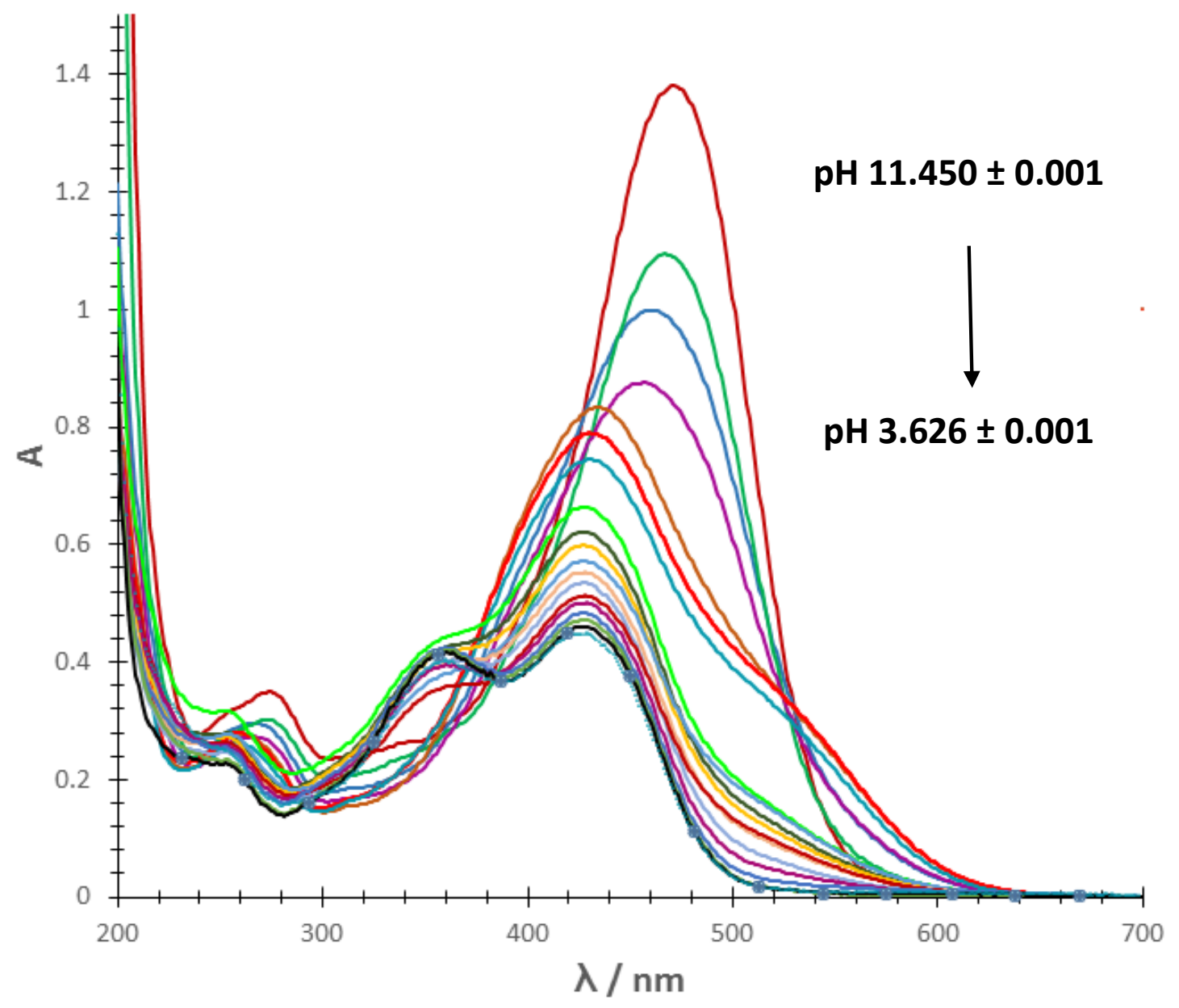

Figura 8. Familia de espectros de absorción para la curcumina [32.5 $\mu \mathrm{M}]$ en disolución acuosa a fuerza iónica a $0.575 \mathrm{M}$ como una función del pH bajo condiciones experimentales obtenidos mediante un análisis puntual. 
Para confirmar la presencia de estos equilibrios y dilucidar cuantos pKas existen y sus respectivos valores se grafica la absorbancia a una o varias longitudes de onda como una función del pH, ver Figura 9. En esta, se aprecia que para cada longitud de onda graficada existe al menos tres posibles puntos de inflexión; por tanto tres pkas. El primero alrededor de $\mathrm{pH}$ 7. Los otros dos, no se distinguen completamente dando la apariencia que se tratara de solo un cambio de pendiente entre los valores de $\mathrm{pH}$ 9-11. Es posible que por la semejanza de los grupos $\mathrm{OH}$ y la simetría de la curcumina, estos cambios en la Figura 7 no se distinguen correctamente. Esto explicaría por qué algunos autores, ver Tabla 3, reportan solo dos valores de constante de acidez.

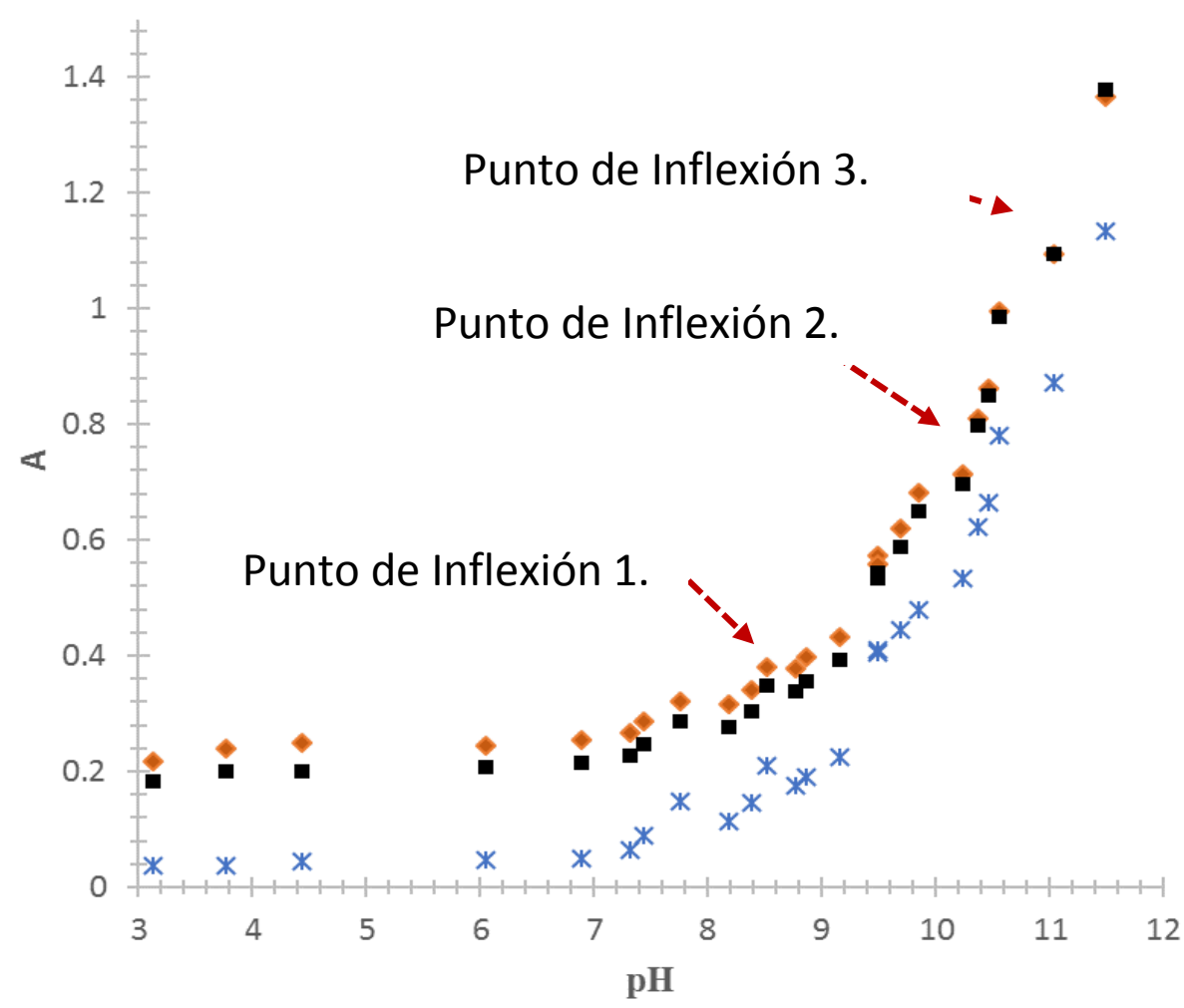

Figura 9. Absorbancia como una función del pH a (४) $465 \mathrm{~nm}$, a (•) $469 \mathrm{~nm}$ y (Ж) $500 \mathrm{~nm}$ para la curcumina $[32.5 \mu \mathrm{M}]$ en disolución acuosa bajo condiciones experimentales controladas.

A pesar de que en este trabajo se estudia a la curcumina bajo condiciones experimentales controladas, determinar el número y los valores exactos con la menor incertidumbre posible resulta una tarea complicada utilizando solamente esta estrategia gráfica. Motivo por el cual se propone aprovechar el programa computacional Stability QUotiens from Absorbance Data, SQUAD, por sus siglas en inglés (ver Apéndice 3).

Según los valores de pka mostrados en la Tabla 3 reportados por distintos autores y de acuerdo a los resultados presentados en este trabajo, para distinguir si la 
curcumina presenta dos o tres valores de pKa se procedió a utilizar primero un modelo de dos equilibrios (Modelo I) y posteriormente se cambió a uno de tres equilibrios (Modelo II). Para ambos modelos, se empleó una $\mathrm{n}=4975$ datos provenientes de 25 espectros de absorción de 5 en $5 \mathrm{~nm}$ abarcando un intervalo de longitud de onda de 201 a $696 \mathrm{~nm}$ en un intervalo de $\mathrm{pH}$ de (11.450 a 3.626) \pm 0.001 .

En la Tabla 4 se muestran los valores de constantes de acidez obtenidos por el programa computacional SQUAD para ambos modelos.

Tabla 4. Valores de constantes de acidez para la curcumina en disolución acuosa según un modelo de dos equilibrios (Modelo I) y un modelo de tres equilibrios (Modelo II).

\begin{tabular}{|cccc|}
\hline Modelo I & pKa \pm o & Modelo II & pKa \pm o \\
\hline $\mathrm{H}_{2} \mathrm{Cur}=\mathrm{HCur}^{-}+\mathrm{H}^{+}$ & $8.843 \pm 0.016$ & $\mathrm{H}_{3} \mathrm{Cur}=\mathrm{H}_{2} \mathrm{Cur}^{-}+\mathrm{H}^{+}$ & $7.428 \pm 0.015$ \\
\hline $\mathrm{HCur}^{-}=\mathrm{Cur}^{2-}+\mathrm{H}^{+}$ & $10.783 \pm 0.024$ & $\mathrm{H}_{2} \mathrm{Cur}^{-}=\mathrm{HCur}^{2-}+\mathrm{H}^{+}$ & $9.552 \pm 0.024$ \\
\hline & & $\mathrm{HCur}^{--}=\mathrm{Cur}^{3-}+\mathrm{H}^{+}$ & $10.946 \pm 0.034$ \\
\hline & & & $\mathbf{U} / \mathbf{n}=\mathbf{3} \mathbf{x 1 0}$ \\
\hline
\end{tabular}

De acuerdo a los protones ácidos disponibles de la curcumina en su forma enólica en disolución acuosa [14, 15], ver Figura 5, y de acuerdo a los resultados de $U / n$ (Tabla 4), donde $U$ es la minimización de suma de cuadrados y $n$ el número de datos del sistema tiene más sentido químico hablar de tres valores de pkas en lugar de dos para explicar el número y el valor de cada constante de acidez de la curcumina en disolución acuosa bajo condiciones experimentales controladas.

Si bien Bernabé et.al. [20] utilizan la misma herramienta computacional para el tratamiento de los datos de absorbancia para el cálculo de las constantes de acidez; es necesario puntualizar que en este trabajo se emplea una versión más actualizada del software SQUAD que permite trabajar con un mayor número de datos de absorbancia. Esto amplia el intervalo de datos con los que se refina el cálculo de pka's y por tanto, optimiza la minimización de suma de cuadrados. Además, en el presente trabajo se logra disminuir la influencia del tiempo sobre la degradación de la curcumina en medio acuoso al utilizar una estrategia puntual para la determinación de los espectros de absorción a cada valor de pH empleado. 
En la Tabla 5 se muestran las diferencias significativas entre el presente trabajo y Bernabé et.al [20].

Tabla 5. Diferencias entre el método de Bernabé et,al. y el presente trabajo.

\begin{tabular}{|c|c|}
\hline $\begin{array}{c}\text { Bernabé } \\
\text { et.al [2004] }\end{array}$ & Este trabajo [2019] \\
\hline $\begin{array}{c}\text { Intervalo de } \mathrm{pH} \text { : } \\
6.475-11.384\end{array}$ & $\begin{array}{c}\text { Intervalo de } \mathrm{pH} \text { : } \\
3.626-11.450\end{array}$ \\
\hline $\begin{array}{l}\text { Una misma disolución de trabajo. } \\
\text { Respuesta dependiente del } \\
\text { tiempo }\end{array}$ & $\begin{array}{l}32 \text { disoluciones de trabajo. Análisis } \\
\text { puntual. } \\
\text { Respuesta independiente del } \\
\text { tiempo }\end{array}$ \\
\hline [Curcumina] $=8.00 \mu \mathrm{M}$ & {$[$ Curcumina $]=32.5 \mu \mathrm{M}$} \\
\hline $\begin{array}{c}\mathrm{n}=1034 \text { datos } \\
238<\lambda / \mathrm{nm}<560 \\
7 \mathrm{~nm} \text { en } 7 \mathrm{~nm}\end{array}$ & $\begin{array}{c}\mathrm{n}=4975 \text { datos } \\
201<\lambda / \mathrm{nm}<696 \\
5 \mathrm{~nm} \text { en } 5 \mathrm{~nm}\end{array}$ \\
\hline
\end{tabular}

Una de las ventajas del programa computacional SQUAD, es que además del cálculo de las constantes de acidez, también devuelve los valores de los coeficientes de absortividad molar de las diferentes especies que absorben en el sistema, ver Figura 10a. Con esta información y tomando en cuenta la concentración de la curcumina es posible calcular los diferentes espectros de absorción en función de la longitud de onda en todo el intervalo de $\mathrm{pH}$. De esta manera, se puede apreciar en la Figura 10b una buena correlación entre los datos experimentales (marcadores) y los datos teóricos (líneas continuas) proporcionando confianza en el método descrito en este trabajo para la determinación de las constantes de acidez de la curcumina. 

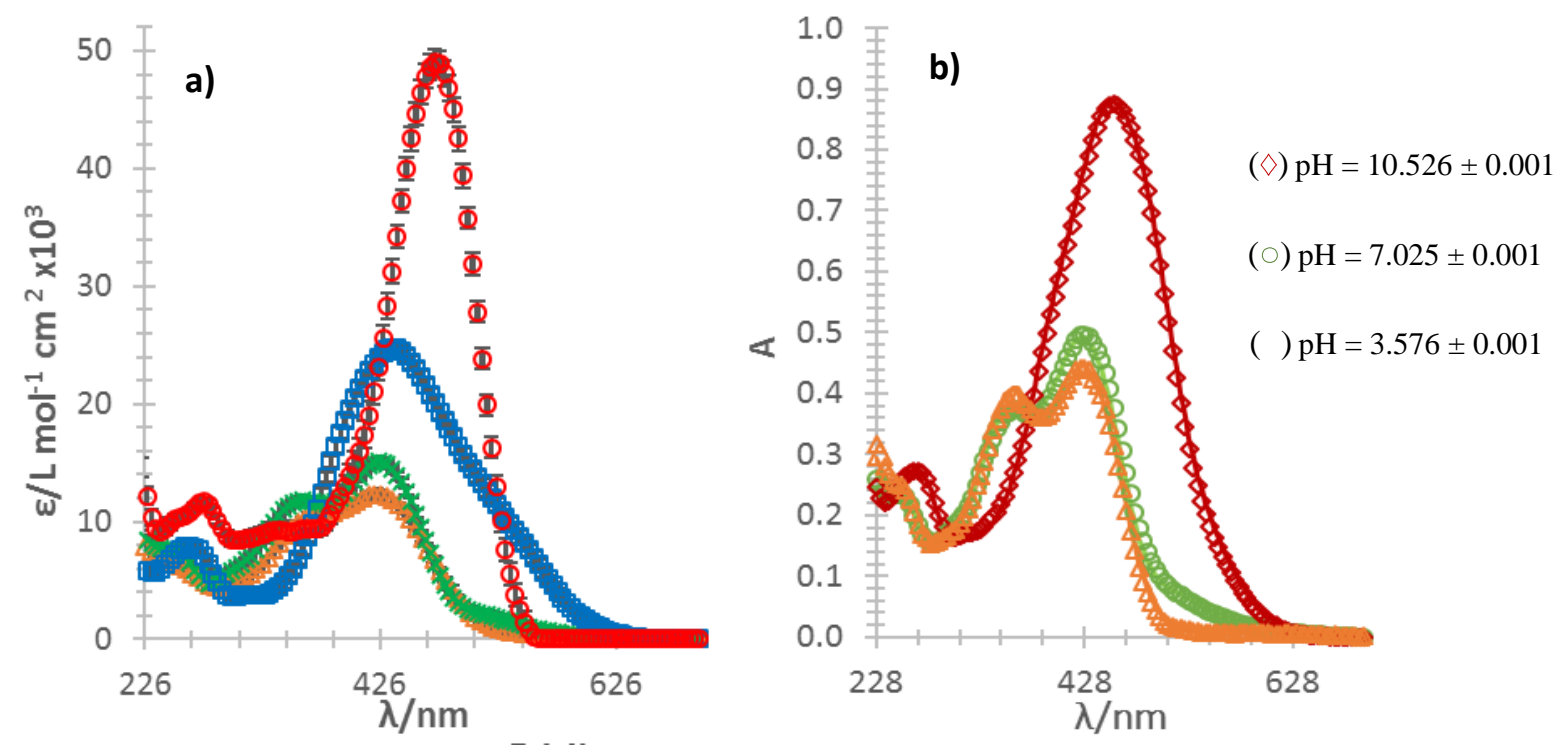

Especie $(\triangle) \mathrm{H}_{3} \mathrm{Cur}(\mathrm{X}) \mathrm{H}_{2} \mathrm{Cur}(\square) \mathrm{HCur}^{2-}(\mathrm{O}) \mathrm{Cur}^{3-}$

Figura 10. a) Coeficientes de absortividad molar de las diferentes especies de la curcumina en disolución acuosa calculados por el programa computacional SQUAD mediante el análisis de los espectros de absorción mostrados en la Figura 8. b) Comparación de algunos espectros de absorción representativos donde los marcadores son los datos experimentales y las lineas continuas son los espectros teóricos.

Con esta información disponible, es posible generar un diagrama de fracción molar que nos permita determinar la fracción molar de cada una de las formas de especiación de la curcumina según el valor de pH (Figura 11). Esto es de gran importancia para proponer y optimizar diseños de medios de reacción para su caracterización en disolución acuosa. Además de proporcionar información relevante del porcentaje de cada forma de especiación de la curcumina en medio acuoso. 

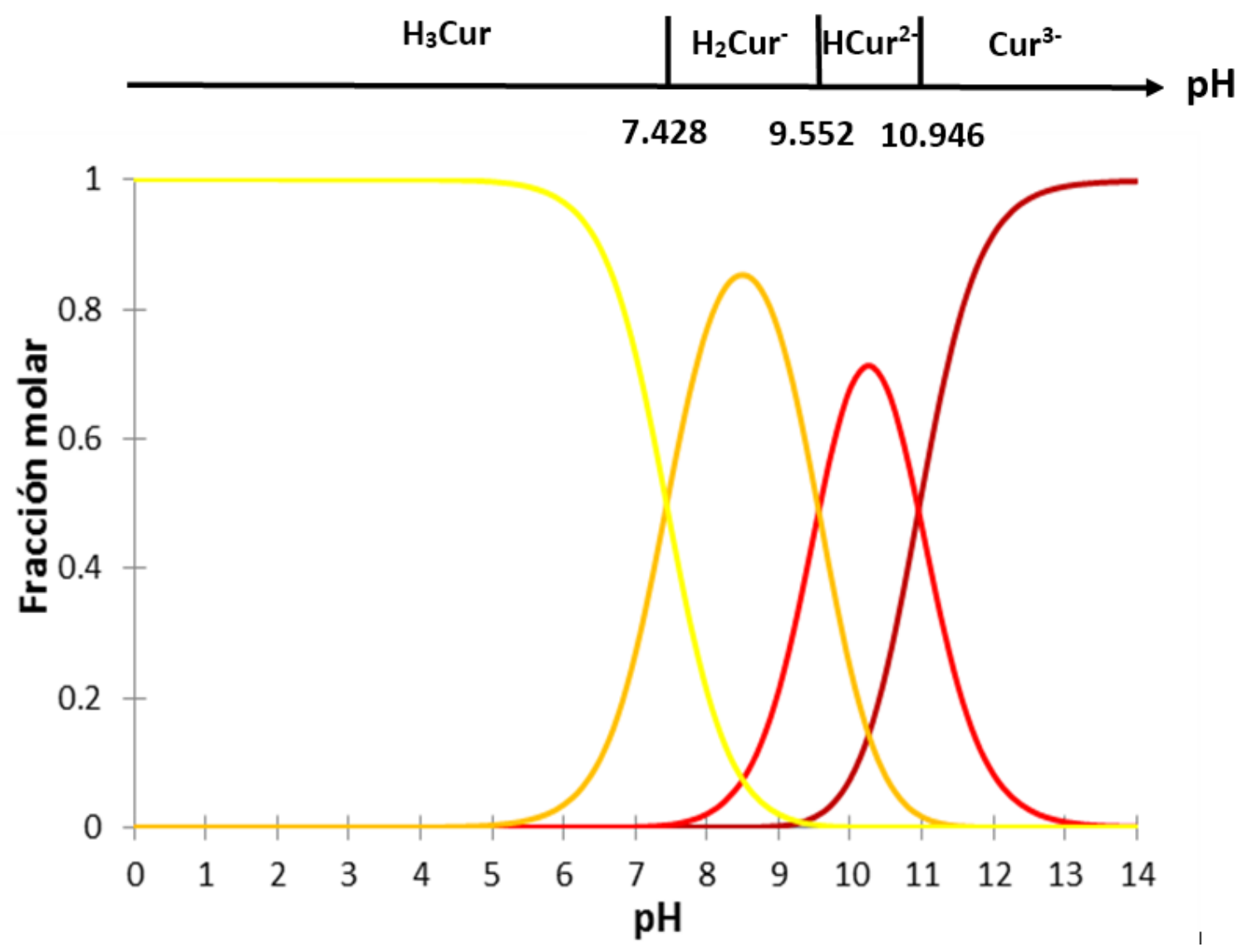

Figura 11. Diagrama de fracción molar de la curcumina en medio acuoso utilizando los valores de pKa (Modelo de tres equilibrios) reportados en este trabajo, ver Tabla 4. 


\subsection{Conclusiones.}

Los valores de constantes de acidez para la curcumina en disolución acuosa calculados en este trabajo son; pka $1=7.428 \pm 0.015$ correspondiente al equilibrio $\mathrm{H}_{3} \mathrm{Cur}=\mathrm{H}_{2} \mathrm{Cur}^{-}+\mathrm{H}^{+}$, pka2 $=9.552 \pm 0.024$ correspondiente a la segunda disociación $\mathrm{H}_{2} \mathrm{Cur}^{-}=\mathrm{HCur}^{2-}+\mathrm{H}^{+}$y pka $=10.946 \pm 0.034$ para la última disociación ácida $\mathrm{HCur}^{2-}=$ $\mathrm{Cur}^{3-}+\mathrm{H}^{+}$. Estos valores de pKa fueron obtenidos bajo condiciones experimentales de ausencia de luz, atmósfera de $\mathrm{N}_{2(\mathrm{~g})}$ y temperatura constante $(21.0 \pm 0.1)^{\circ} \mathrm{C}$ mediante un análisis puntual de cada disolución de trabajo minimizando así el aporte de la degradación de la curcumina en disolución acuosa ofreciendo mejores resultados en términos estadísticos con respecto a otros autores.

Así mismo, se determinaron, los valores de los coeficientes de absortividad molar para cada una de las especies que absorben en el sistema utilizando el software SQUAD lo cual permite conocer la especiación de la curcumina en disolución acuosa siendo así el ion curcuminato la especie que mayor absorbe. 


\section{CAPÍTULO 3. Estrategias de estabilización para la Curcumina en medio acuoso.}

"La teoría guía pero el experimento decide" - Izaak M. Kolthoff 


\subsection{Estabilización química de la curcumina mediante la formación de un compuesto de coordinación con Zn (II) en medio acuoso.}

Se ha propuesto en la literatura que la posición de la 1,3 dicetona de la curcumina está en un equilibrio tautomérico de tipo ceto-enol el cual puede reaccionar con los iones metálicos presentes en el medio para formar un complejo de coordinación y así ofrecer estabilidad a la curcumina. Bajo este esquema diversos autores han reportado la interacción entre curcumina-metal. Bernabé et. al. [31] demuestran la interacción entre la curcumina y el Fe (III)/Fe (II) en medio acuoso. Barik et. al. [32] sugieren un modelo con el Cu (II) de tipo ML. Krishnankutty et. al. [3334] estudian la interacción del $\mathrm{Cu}$ (II) y Zn (II) con la curcumina proponiendo un modelo de tipo ML2. Baum et. al. [35] apoyan el modelo de tipo ML2 en presencia de $\mathrm{Cu}$ (II) y sugiere que la interacción entre curcumina y $\mathrm{Zn}$ (II) es débil. Sin embargo, estos estudios son en disolventes no acuosos o en su defecto, fueron modelados mediante química computacional. Adicionalmente no reportan ninguna constante de formación entre el metal y la curcumina a excepción de Bernabé et. al. [31].

Por lo tanto, se propone para este trabajo la posibilidad de formar un compuesto de coordinación entre el Zinc (II) y la curcumina con la finalidad de explorar su utilidad para aumentar la estabilidad de la curcumina en medio acuoso que si bien en el Capítulo 1 se demuestra que se minimiza la velocidad de degradación de la curcumina mediante el control de las condiciones experimentales, no queda duda que aún presenta una degradación significativa en medios ácidos.

\subsubsection{Reactivos.}

Curcumina grado analítico marca Sigma Aldrich ( $\geq 99.5 \%$ ), lentejas de $\mathrm{NaOH}$ marca Macron, $\mathrm{HClO}_{4}$ concentrado, Sulfato de Zinc ( $\left.\geq 100 \%\right)$ marca J. T. Baker.

\subsubsection{Equipo.}

La lectura de $\mathrm{pH}$ se realiza con un potenciómetro marca sensION equipado con un electrodo de vidrio de $\mathrm{Ag}^{\circ} \mid \mathrm{AgCl}[\mathrm{KCl}, 3 \mathrm{M}]$ marca Hach $5014 \mathrm{~T}$.

Para el control de la temperatura se utiliza un recirculador Grant W14.

Cada espectro de absorción se registra mediante un espectrofotómetro Lambda 20 UV Vis equipado con celdas de cuarzo $1 \mathrm{~cm}$ de longitud de paso óptico. El intervalo de adquisición de los espectros es de 200 a $700 \mathrm{~nm}$ a una velocidad de 1960 $\mathrm{nm}$ min $^{-1}$. 
Para el registro de las curvas de corriente como una función del potencial se utiliza una celda convencional con capacidad para tres electrodos siendo el electrodo de pasta de carbono, el electrodo de trabajo preparado de acuerdo al método de Ramírez-Silva [28, 29]. Se utiliza un electrodo de referencia de $\mathrm{Ag}^{\circ} \mid \mathrm{AgCl}[\mathrm{KCl}, 3 \mathrm{M}]$ marca BAS MF-2052 y un alambre de platino BAS MW-1032 como contraelectrodo. Los registros voltamperométricos se hacen utilizando una ventana de potencial de $350 \mathrm{mV}$ a $1000 \mathrm{mV}$, a una velocidad de barrido de $100 \mathrm{mV} \mathrm{s}^{-1}$ y considerando un ciclo de barrido hacia sentido anódico.

\subsubsection{Método.}

En una celda de trabajo se mantiene una disolución de curcumina [32.5 $\mu \mathrm{M}$ ] bajo condiciones de amortiguamiento de $\mathrm{pH} 3.576 \pm 0.001$ y se realizan adiciones independientes de una disolución stock de $\mathrm{Zn}(\mathrm{II})[0.01 \mathrm{M}]$. Se exploraron tres relaciones molares: [Zn (II)] / [Cur] = 0.5, [Zn (II)] / [Cur)] = 1.0 y [Zn (II)] / [Cur] = 2.0. Para cada relación se adquiere el espectro de absorción por UV-Vis y el voltamperograma cíclico respectivo.

Entre cada barrido se renovó la superficie del electrodo de trabajo mediante pulición sobre un papel.

Es importante mencionar que en todo el tiempo que duró el experimento se mantuvieron condiciones experimentales controladas (mencionadas en el capítulo 1).

\subsubsection{Estudio espectro-electroquímico de la interacción Curcumina-Zn (II) a pH} $3.576 \pm 0.001$.

En la Figura 12 se muestra la respuesta de absorbancia respectiva a cada relación molar bajo estudio.

De acuerdo al inserto de la Figura 12 (izquierda), el máximo de absorción a 423 $\mathrm{nm}$ y la banda localizada a $263 \mathrm{~nm}$ disminuye conforme aumenta la concentración de Zn (II). Estos cambios en la respuesta de absorbancia (cambios en las transiciones electrónicas) son ocasionados por la interacción de la curcumina con el Zn (II) mediante la formación de un posible compuesto de coordinación. Sin embargo, como quedó demostrado en el Capítulo 1, a pH $3.576 \pm 0.001$, la $\mathrm{V}_{\text {deg }}$ es de $(2.86 \pm 0.29) \%$ DC min $^{-1}$ lo cual abre la posibilidad que lo que se esté registrando como respuesta de absorbancia sea un aporte del compuesto coordinado formado y la degradación de la curcumina misma. Para descartar esta posibilidad se registran los voltamperogramas cíclicos de la curcumina en medio acuoso toda vez que si el Zn (II) se coordina a la 
curcumina, se esperarían cambios de potencial o de corriente con respecto a las señales voltamperométricas de la curcumina en ausencia de metal.

En la Figura 12 (derecha) se muestran los desplazamientos hacia valores

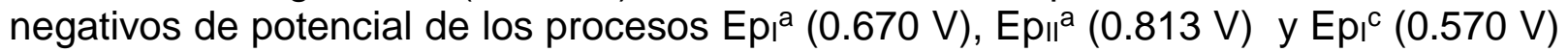
conforme incrementa la concentración de Zn (II) en disolución acuosa. Adicional a este desplazamiento de potenciales a valores más reductores se presenta una caída de corriente de los picos anódico como catódico. Si a tiempo cero se tiene la curcumina en su forma reducida, Curcuminared, se esperaría que de formar complejos de coordinación con el Zn (II) la fuerza del oxidante, Curcuminaox, incremente traduciéndose en un aumento en el valor de potencial lo cual no sucede. De hecho, lo que se ve es un desplazamiento de los valores de potencial hacia valores negativos (incrementa la fuerza del reductor), lo cual confirma que lo que se está registrando es una interacción entre el Zn (II) y Curcuminaox, es decir la curcumina ya oxidada. Por tanto, existe una competencia entre la formación de los sistemas: Curcuminared-Zn(II), Curcuminaox-Zn(II), y la degradación de la curcumina misma. Además, el tener a la curcumina totalmente protonada a valores de $\mathrm{pH}$ ácidos disminuye las posibilidades de los sitios de coordinación entre el Zn (II) y la curcumina.

Con el objetivo de explorar si el sistema coordinado: Curcuminared-Zn(II) se favorece a valores de $\mathrm{pH}$ ligeramente altos se propone hacer el mismo estudio pero a $\mathrm{pH} 7.025 \pm 0.001$. Toda vez que la $\mathrm{V}_{\text {deg }}$ a este $\mathrm{pH}$ es de $(0.47 \pm 0.03) \% \mathrm{DC} \mathrm{min}^{-1}$. Lo cual no solo minimiza el aporte de los productos de degradación de la curcumina, sino además elimina la influencia de los hidroxocomplejos del Zn (II) característicos de valores de $\mathrm{pH}$ alcalinos.
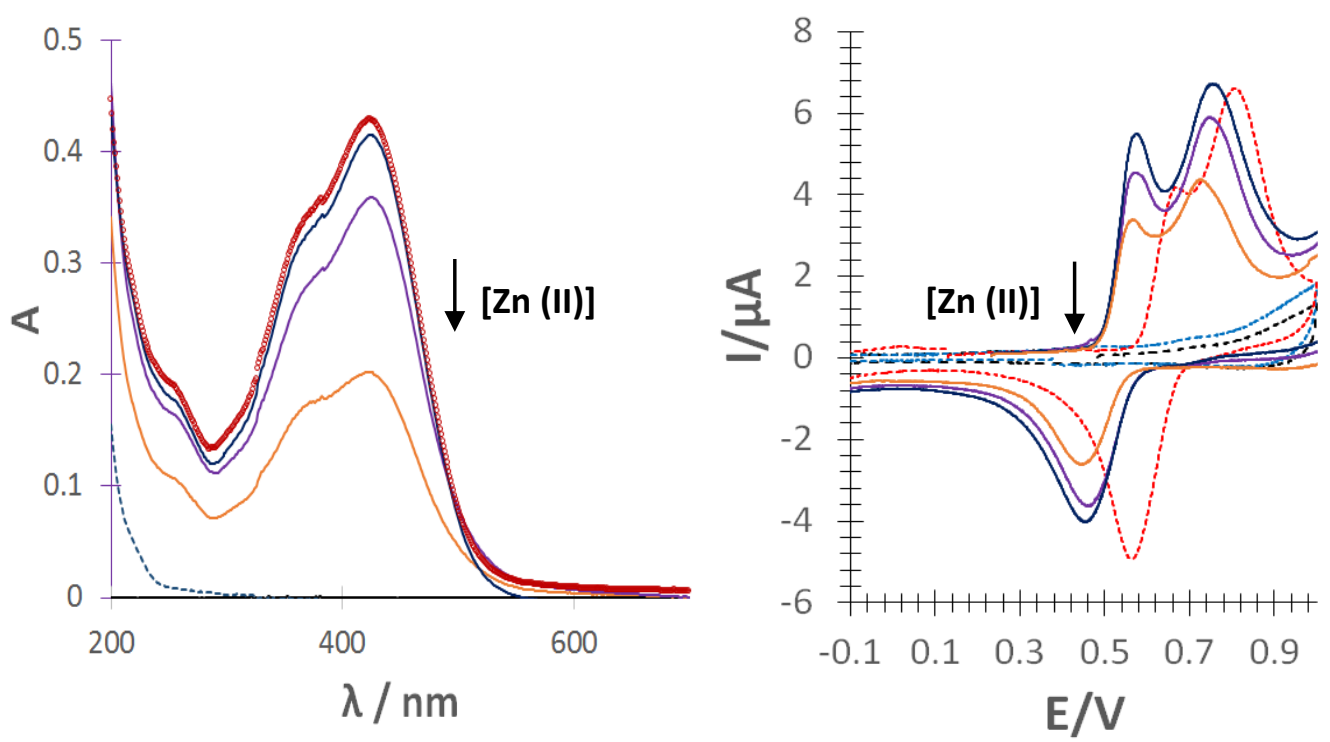

Figura 12. Izquierda: Familia de espectros de absorción de la curcumina en presencia del Zn (II) a diferentes relaciones molares. Derecha: Voltamperogramas cíclicos de la curcumina en presencia del $\mathrm{Zn}$ (II) a diferentes relaciones molares. Valor de pH $3.576 \pm 0.001$ bajo condiciones experimentales controladas. (-) [Zn (II)] / [Cur] = 0.5, (-) [Cur] / [Zn (II)] = 1.0 y (-) [Zn (II)] / [Cur] = 2.0, (---) $\mathrm{H}_{3} \mathrm{Cur},(---)$ $\mathrm{Zn}(\mathrm{II})$. 


\subsubsection{Estudio espectro-electroquímico de la interacción Curcumina-Zn (II) a pH}

$7.025 \pm 0.001$.

Siguiendo la misma estrategia que en el punto 3.1.4 se hace el estudio espectro-electroquímico de la curcumina a diferentes relaciones molares con el Zn (II) con el objetivo de evidenciar la interacción entre ambas especies a través de la formación de un complejo de coordinación.

En la Figura 13, se muestran la familia de espectros de absorción (izquierda) y los voltamperogramas cíclicos (derecha) obtenidos a diferentes relaciones molares de curcumina y Zn (II) en disolución acuosa a pH 7.025 \pm 0.001 .

Si el Zn (II) interacciona con la curcumina mediante un mecanismo de formación de un compuesto de coordinación, las transiciones electrónicas asociadas a la curcumina se deben modificar y con ello la respuesta de absorbancia. Para que esto suceda, los grupos - OH (ver Figura 1) que son las zonas suceptibles donde el Zn (II) puede coordinarse deben estar desprotonadas. De acuerdo a la Figura 11, el diagrama de fracción molar muestra que a este valor de $\mathrm{pH}$ se tiene la siguiente especiación de curcumina: $\phi^{\mathrm{H} 3 \mathrm{Cur}}=71.62 \%, \phi^{\mathrm{H} 2 \mathrm{Cur}(2-)}=28.29 \%, \phi^{\mathrm{HCur}(-)}=0.08 \%$ y $\phi^{\text {Cur( }(3-)}=0.00 \%$. Con ello se confirma que al menos existe un porcentaje de casi $30 \%$ de la curcumina sin un protón en disolución acuosa favoreciendo así las condiciones para la interacción con el Zn (II). Dado que el protón del grupo ceto-enol puede estar comprometido por la presencia de puentes de hidrógeno se espera que el -OH que se propone (según el diagrama de zonas de predominio, ver Figura 11) que esté desprotonado pertenezca a cualquiera de los dos grupos fenólicos de la curcumina. De ser así se esperaría un cambio en la banda a $263 \mathrm{~nm}$, asociada a transiciones $\pi$ a $\Pi^{*}$, lo cual se puede observar en la Figura 13 (Izquierda).
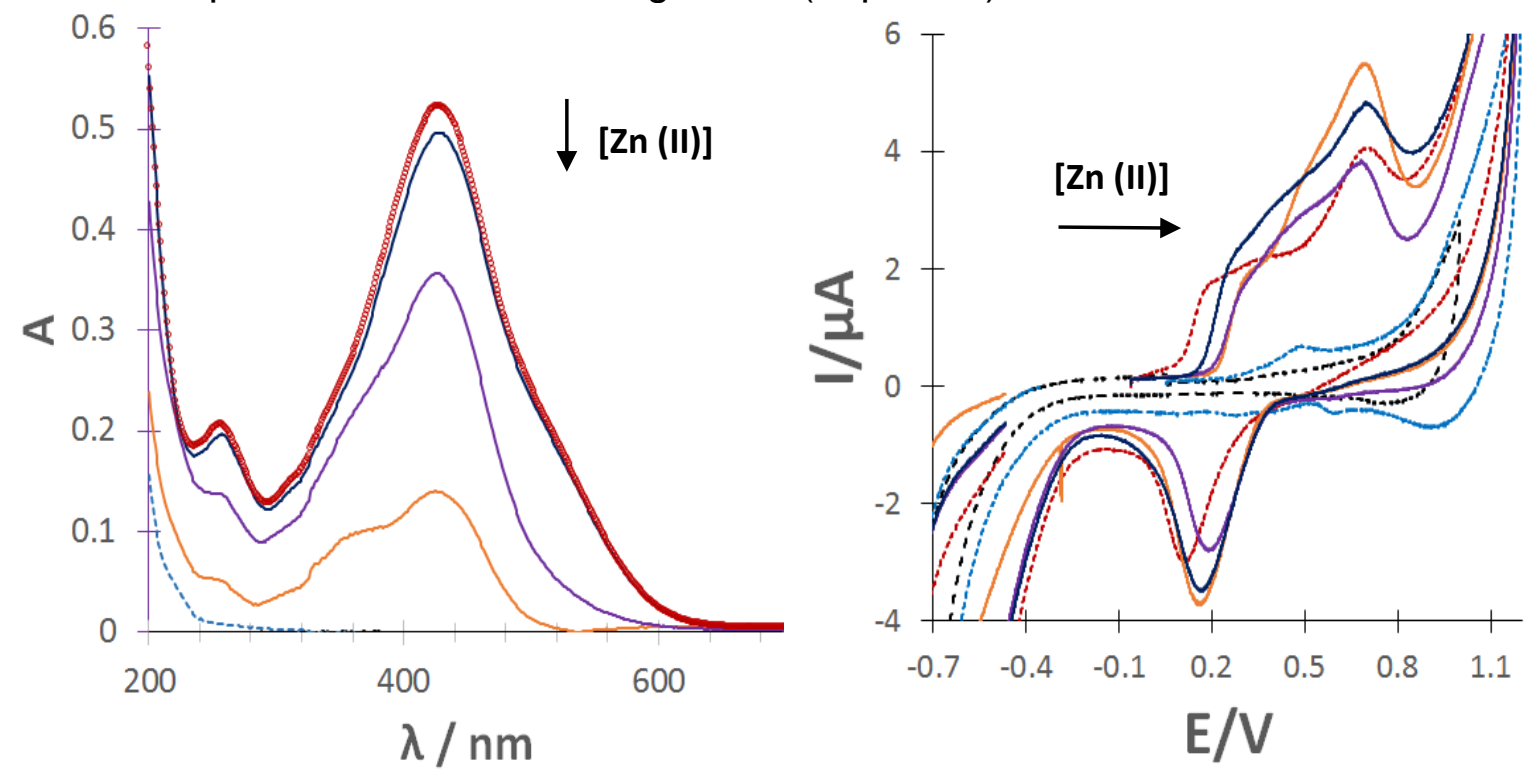

Figura 13. (Izquierda) Familia de espectros de absorción de la curcumina en presencia del Zn (II) a diferentes relaciones molares. (Derecha) Voltamperogramas cíclicos de la curcumina en presencia del Zn (II) a diferentes relaciones molares. Valor de $\mathrm{pH} 7.025 \pm 0.001$ bajo condiciones experimentales controladas. (-) [Zn (II)] $/[\mathrm{Cur}]=$ 0.5, (-) [Zn] / [Cur] = 1.0 y (-) [Zn (II)] / [Cur] = 2.0, (---) $\mathrm{H}_{3} \mathrm{Cur},(--) \mathrm{Zn}(\mathrm{II})$. 
Conforme aumenta la concentración de Zn (II) la absorbancia a 263 nm disminuye así como el máximo de absorción a $423 \mathrm{~nm}$. Para corroborar la propuesta de que el $\mathrm{Zn}$ (II) se coordina por cualquiera de los grupos - $\mathrm{OH}$ localizados en el extremo de la molécula se hace un estudio voltamperométrico.

Si a pH $7.025 \pm 0.001$ se minimiza el aporte de la degradación de la curcumina, y además el Zn (II) según el estudio por espectrofotometría de UV-Vis se coordina en los sitios susceptibles de oxidación, es decir; donde hay grupos - $\mathrm{OH}$; entonces se debería favorecer el sistema Curcuminared-Zn(II). Esto supondría un aumento en la fuerza del oxidante, Curcuminaox, conforme aumenta la concentración de Zn (II) y por tanto, un incremento en los valores de potencial. Aunque, a pH $7.025 \pm 0.001$, los procesos de oxidación están sobrepuestos por la mezcla de especies de curcumina se logra demostrar en la Figura 13 (Derecha) que conforme incrementa la concentración del Zn (II) en el sistema, el valor de potencial del primer proceso de oxidación localizado a $0.200 \mathrm{~V}$ se recorre a valores de potencial positivos. Es decir, se confirma el aumento de la fuerza del oxidante, Curcuminaox, a causa de la formación del sistema coordinado Curcuminared-Zn(II).

Considerando que la respuesta voltamperométria entre las relaciones molares [Zn] / [Cur] = 1.0 y [Zn (II)] / [Cur] = 2.0 prácticamente es la misma, se propone que la relación molar del compuesto coordinado que se forma es 1 a 1, de un Zn (II) por una curcumina.

De manera esquemática se describe el modelo propuesto anteriormente y que explica los cambios en la respuesta voltamperométrica a pH $7.025 \pm 0.001$.

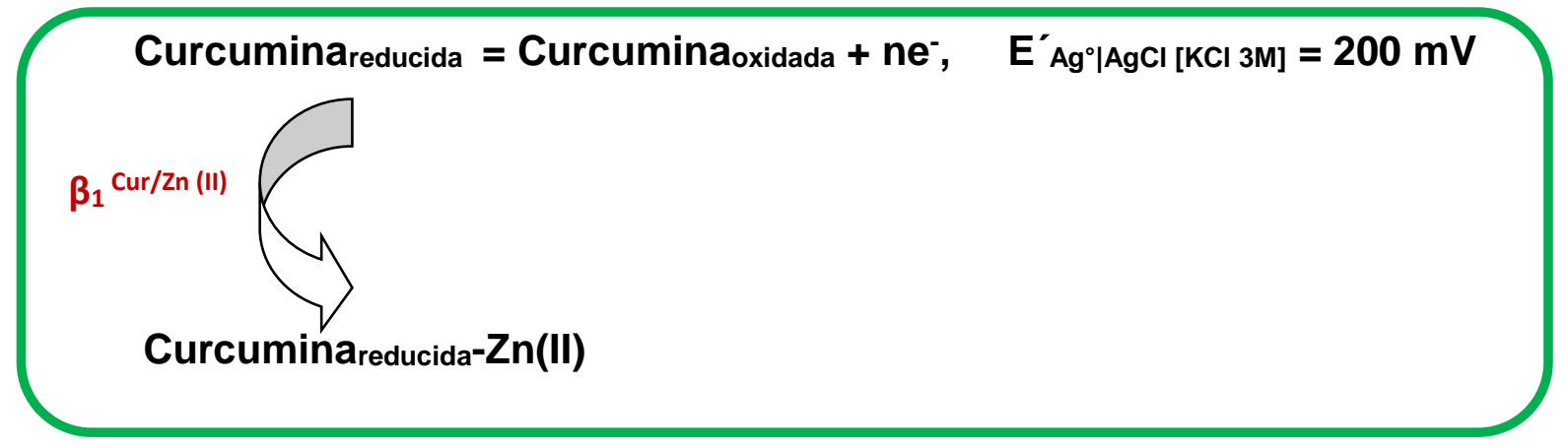




\subsubsection{Conclusiones.}

Se demuestra que existe una interacción entre la curcumina y el ion metálico $\mathrm{Zn}$ (II) en disolución acuosa mediante un mecanismo de formación de un compuesto de coordinación Curcuminared-Zn(II). Esta interacción se favorece a pH $7.025 \pm 0.001$ con respecto a $\mathrm{pH} 3.576 \pm 0.001$.

Si bien se demuestra que se favorecen estos compuestos a pH $7.025 \pm 0.001$ controlando las condiciones experimentales, las señales voltamperométricas resultan complicadas de interpretar por tanto esta estrategia para mejorar la estabilidad de la curcumina en medio acuoso resulta poco viable.

Por tanto, a continuación se propone hacer un estudio de estabilidad de la curcumina mediante la formación de compuestos de inclusión con $\beta$-ciclodextrina a pH $3.576 \pm 0.001$. 


\subsection{Estabilización química de la curcumina mediante la formación de un compuesto de inclusión con $\beta$-Ciclodextrina.}

Las ciclodextrinas son oligómeros cíclicos de $\alpha$-D-glucosa obtenidas a partir de la acción enzimática sobre el almidón [36]. Las ciclodextrinas han sido ampliamente usadas en especial por su habilidad para formar complejos de inclusión con una gran variedad de moléculas denominadas "huésped". Por consecuencia, se tiene en la mayoría de los casos una repercusión en el aumento de la solubilidad, la estabilidad química, biodisponibilidad y muchas veces una protección contra eventos que puedan ocasionar su oxidación [37-39].

Comercialmente existen tres principales presentaciones de ciclodextrina: la $\alpha$ ciclodextrina $(\alpha-C D)$, la $\beta$-ciclodextrina $(\beta-C D)$ y la $\gamma$-ciclodextrina $(\gamma-C D)$. En la Tabla 6 se resumen algunas características importantes de estas.

Tabla 6. Propiedades fisicoquímicas más relevantes de las ciclodextrinas.

\begin{tabular}{|c|c|c|c|}
\hline Propiedad & $\alpha-C D$ & $\beta-C D$ & $\gamma-\mathbf{C D}$ \\
\hline $\begin{array}{l}\text { No. de unidades de } \\
\text { glucosa }\end{array}$ & 6 & 7 & 8 \\
\hline $\begin{array}{l}\text { Fórmula empírica } \\
\text { (anhidra) }\end{array}$ & $\mathrm{C}_{36} \mathrm{H}_{60} \mathrm{O}_{30}$ & $\mathrm{C}_{42} \mathrm{H}_{70} \mathrm{O}_{35}$ & $\mathrm{C}_{48} \mathrm{H}_{80} \mathrm{O}_{40}$ \\
\hline $\begin{array}{l}\text { Masa molecular } \\
\quad\left(\mathrm{g} \mathrm{mol}^{-1}\right)\end{array}$ & 972.85 & 1134.98 & 1297.14 \\
\hline $\begin{array}{c}\text { Diámetro de la } \\
\text { cavidad }(\AA)\end{array}$ & 5.2 & 6.6 & 8.4 \\
\hline pka $\left(25^{\circ} \mathrm{C}\right)$ & 12.33 & 12.20 & 12.08 \\
\hline
\end{tabular}

La curcumina a pH $3.576 \pm 0.001$ presenta un porcentaje de degradación aproximado del $70 \%$ en los primeros 12 minutos sin ningún control experimental el cual es disminuido aproximadamente hasta un $30 \%$ en el mismo intervalo de tiempo si y solo sí se mantienen los cuidados experimentales que en el Capítulo 1 se describen. 
Por esta razón, en este capítulo se pretende abordar el efecto de la $\beta-C D$ sobre la estabilidad de la curcumina en medio acuoso bajo dos condiciones experimentales: a) Sin control y b) bajo condiciones controladas.

\subsubsection{Reactivos.}

Curcumina grado analítico marca Sigma Aldrich ( $\geq 99.5 \%)$ sin ningún proceso adicional de purificación, $\beta$-ciclodextrina $(\beta-C D)$ marca Sigma Aldrich ( $\geq 97.0 \%$ ), lentejas de $\mathrm{NaOH}$ marca Macron, $\mathrm{HClO}_{4}$ concentrado.

\subsubsection{Equipo.}

La lectura de $\mathrm{pH}$ se realiza con un potenciómetro marca sensION equipado con un electrodo de vidrio de $\mathrm{Ag}^{\circ} \mid \mathrm{AgCl}[\mathrm{KCl}, 3 \mathrm{M}]$ marca Hach $5014 \mathrm{~T}$.

Para el control de la temperatura se utiliza un recirculador Grant W14.

Cada espectro de absorción se registra mediante un espectrofotómetro Lambda 20 UV Vis equipado con celdas de cuarzo $1 \mathrm{~cm}$ de longitud de paso óptico. El intervalo de adquisición de los espectros es de 200 a $700 \mathrm{~nm}$ a una velocidad de 1960 $\mathrm{nm} \min ^{-1}$.

Para la adquisición de los registros voltamperométricos se utiliza una celda convencional con capacidad para tres electrodos siendo el electrodo de pasta de carbono, el electrodo de trabajo; preparado de acuerdo al método de Ramírez-Silva $[28,29]$. Se utiliza un electrodo de referencia de $\mathrm{Ag}^{\circ} \mid \mathrm{AgCl}[\mathrm{KCl}, 3 \mathrm{M}]$ marca BAS MF2052 y un alambre de platino BAS MW-1032 como contraelectrodo. Los registros voltamperométricos se hacen utilizando una ventana de potencial de $-350 \mathrm{mV}$ a 1000 $\mathrm{mV}$, a una velocidad de barrido de $100 \mathrm{mV} \mathrm{s}^{-1}$ y considerando un ciclo de barrido hacia sentido anódico.

\subsubsection{Método.}

Monitoreo de la estabilidad de la curcumina por espectrofotometría de UV-Vis en presencia de $\beta-C D$. Se monitorea la respuesta de absorbancia de una disolución de trabajo de curcumina [32.5 $\mu \mathrm{M}$ ] preparada previamente en $\mathrm{NaOH}$ [5mM], (mantenida en refrigeración, ver Apéndice 1) en presencia de 4 diferentes concentraciones de $\beta$-CD: I) $0.0 \mathrm{mM}$, II) $0.60 \mathrm{mM}$, III) $10.0 \mathrm{mM}$ y IV) $16.6 \mathrm{mM}$ a un valor de $\mathrm{pH}: 3.576 \pm 0.001$. Estos registros se hacen de manera independiente y bajo dos condiciones experimentales: a) sin control y b) control. 
Monitoreo de la estabilidad de la curcumina por voltamperometría cíclica en presencia de $\beta-C D$. Se monitorea la respuesta de corriente temporal de una disolución de trabajo de curcumina [32.5 $\mu \mathrm{M}]$ preparada previamente en $\mathrm{NaOH}[5 \mathrm{mM}]$ en presencia de $\beta-C D[10.0 \mathrm{mM}]$ a un valor de $\mathrm{pH}: 3.576 \pm 0.001$. Estos registros se hacen de manera independiente bajo condiciones experimentales sin control y con control (estudiadas en el Capítulo 1). Antes de cada registro voltamperométrico se renovó la superficie del electrodo de trabajo mediante el pulimiento sobre papel.

3.2.4 Estudio de la influencia de las condiciones experimentales sobre la estabilidad de la curcumina en medio acuoso en presencia de diferentes concentraciones de $\beta$ CD por espectrofotometría de UV-Vis.

En la Figura 14 se muestran los espectros de absorción para una disolución de curcumina [32.5 $\mu \mathrm{M}$ ] en presencia de 4 diferentes concentraciones de $\beta$-CD: I) 0.0 $\mathrm{mM}$, II) $0.6 \mathrm{mM}$, III) $10.0 \mathrm{mM}$ y IV) $16.6 \mathrm{mM}$ a un valor de $\mathrm{pH}: 3.576 \pm 0.001$ sin control experimental (a, c, e y g) y bajo condiciones experimentales controladas (b, d, f y h).

Se aprecia que al tener una concentración de $\beta$-CD de $0.6 \mathrm{mM}, 10 \mathrm{mM}$ y 16.6 $\mathrm{mM}$ el valor de absorbancia incrementa en los primeros 7 minutos y a partir de ese máximo existe una caída temporal de la respuesta de absorbancia. Se propone que en estos primeros 7 minutos existe un rearreglo entre la curcumina (huésped) y la $\beta$ CD (anfitrión) en disolución acuosa para formar un complejo de inclusión. Formado el complejo, empieza el proceso de degradación de curcumina provocando así la caída de la respuesta de absorbancia.

I) $[\beta-C D] 0.0 \mathrm{mM}$

a)

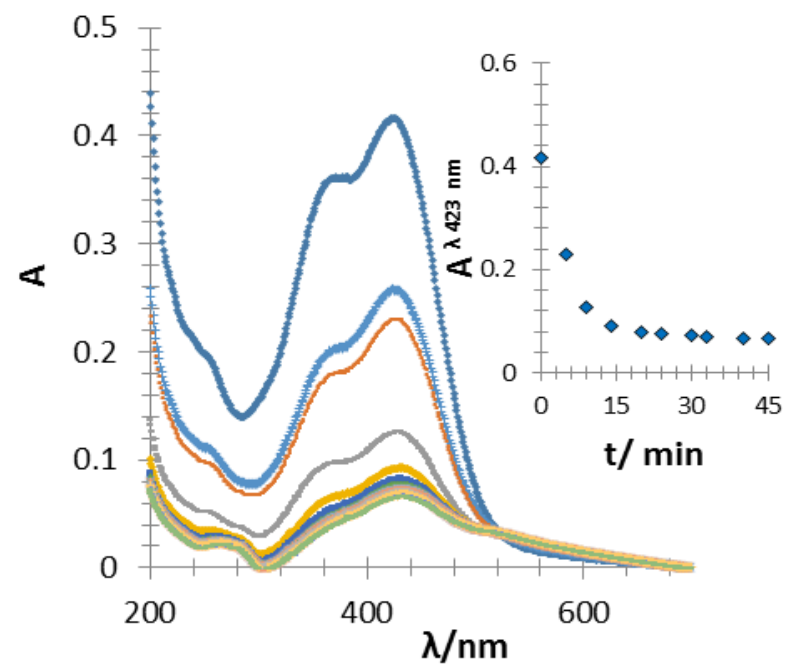

b)

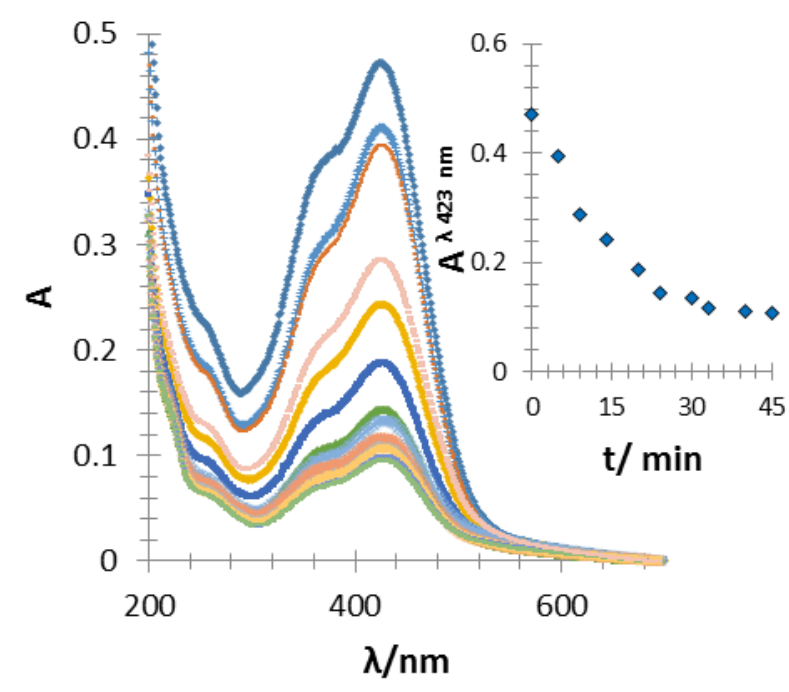


II) $[\beta-C D] 0.6 \mathrm{mM}$

c)

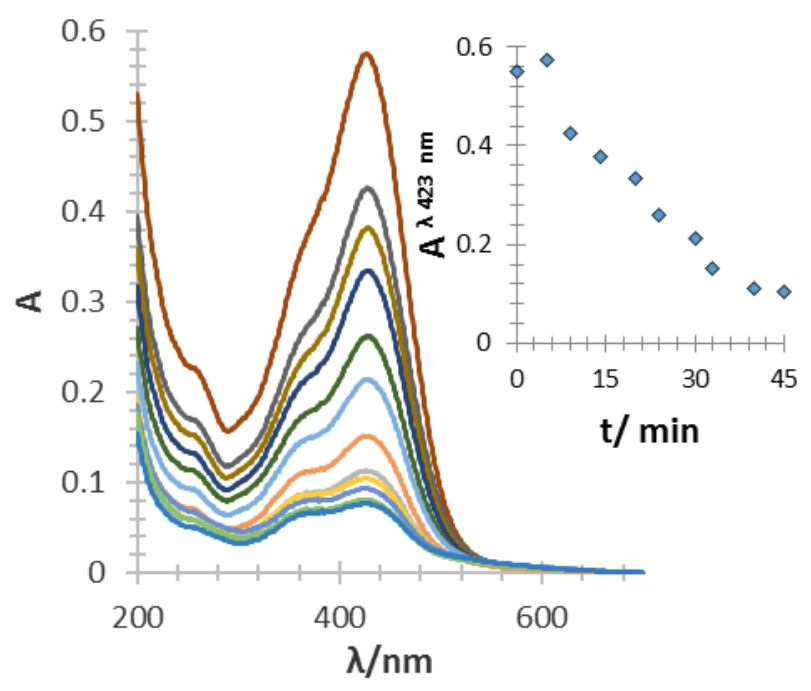

III) $[\beta-C D] 10.0 \mathrm{mM}$

e)

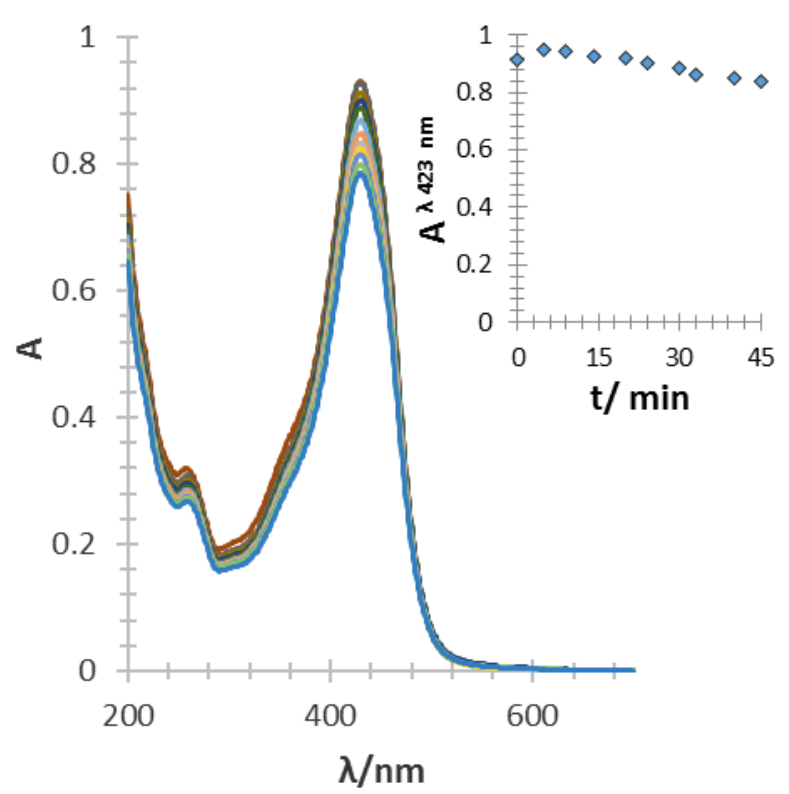

d)

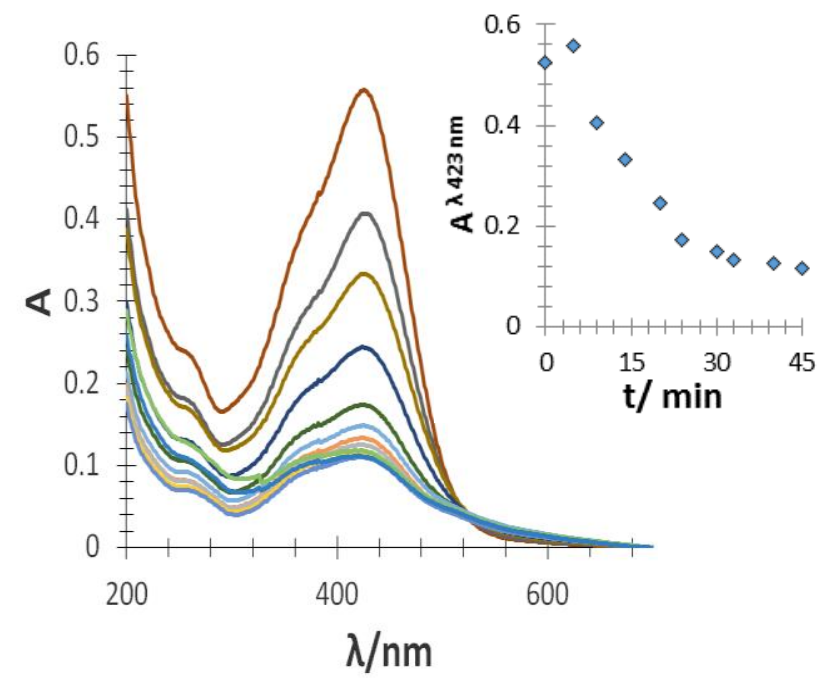

f)

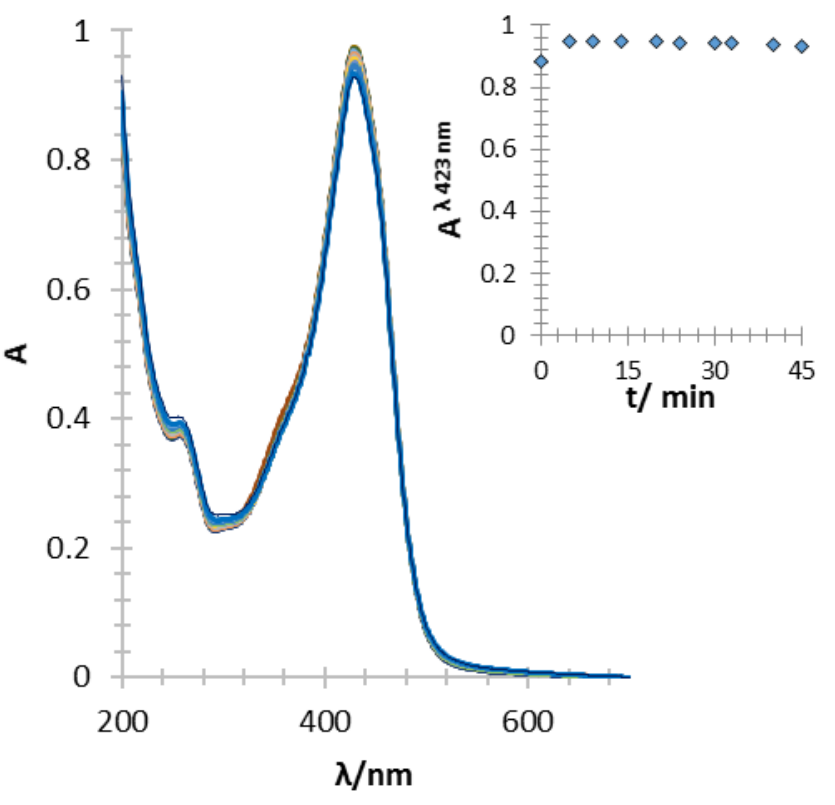




\section{IV) $[\beta-C D] 16.6 \mathrm{mM}$}

g)

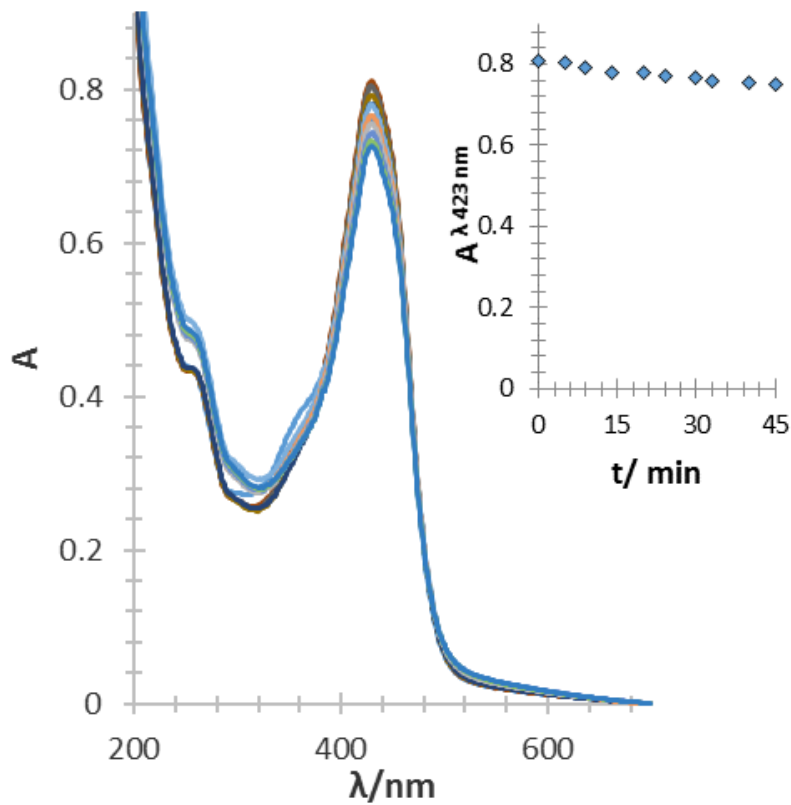

h)

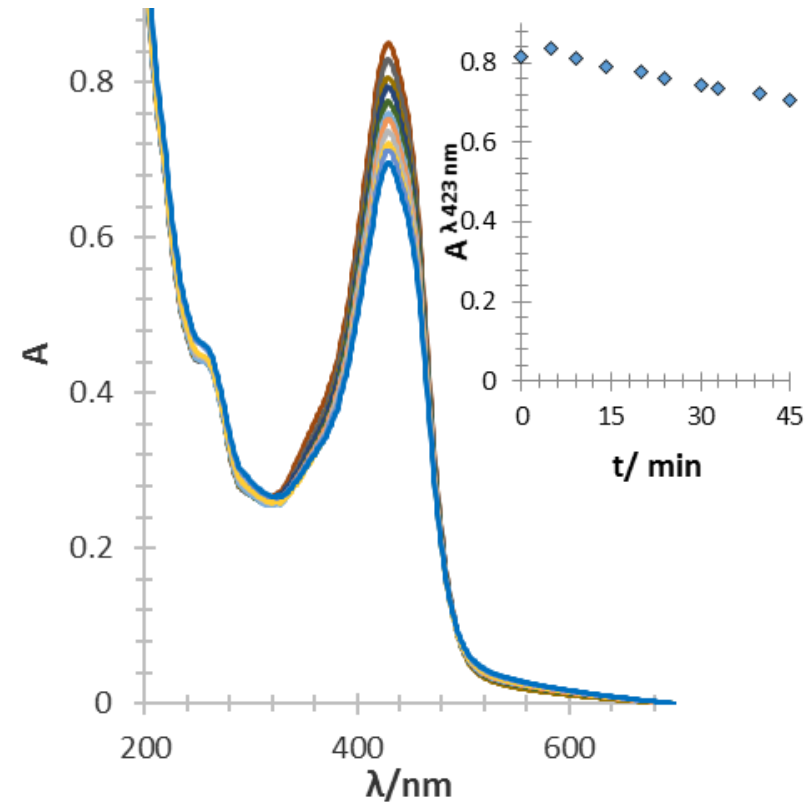

Figura 14. Familia de espectros de absorción de una disolución acuosa de curcumina [32.5 $\mu \mathrm{M}]$ a diferentes concentraciones de $\beta-C D$ a un valor de $\mathrm{pH} 3.576 \pm 0.001$. Sin control (a, c, e y g), y controlando las condiciones experimentales (b, $d, f$ y $h$ ). Los insertos muestran la variación temporal de la absorbancia a $423 \mathrm{~nm}$.

De acuerdo a la Figura 14 se puede determinar que a una concentración en disolución de $\beta-C D$ de $0.60 \mathrm{mM}$ desaparece la banda a $357 \mathrm{~nm}$ lo cual refleja una interacción entre curcumina y $\beta-C D$; sin embargo, la caída de la respuesta de absorbancia todavía es significativamente alta (ver Figura 14 la - Ild). A una concentración de $10 \mathrm{mM}$ de $\beta-C D$ se puede apreciar que el porcentaje de degradación de la curcumina también es muy alto, sobrepasando el $15 \%$ en tan solo 20 minutos y llegando al $62 \%$ en 50 minutos cuando no hay cuidados experimentales. Este porcentaje, en presencia de $10 \mathrm{mM}$ de $\beta$-CD se ve notablemente disminuido cuando se cuidan las condiciones experimentales dado que en 50 minutos la respuesta de absorbancia no presenta un cambio mayor al $3 \%$ lo cual da indicios del aporte que la $\beta-C D$ ofrece a la estabilidad de la curcumina. Así mismo, se puede apreciar que la banda de absorción localizada a $357 \mathrm{~nm}$ desaparece lo cual es un reflejo de la posible interacción entre la curcumina y la $\beta-C D$.

Cuando se trabaja a una concentración de $16.6 \mathrm{mM}$ de $\beta$-CD existe una caída del máximo de absorción $\left(\lambda^{423} \mathrm{~nm}\right)$ del $8 \%$ después de 50 minutos transcurridos. Existe la posibilidad que a esta concentración de $\beta$-CD los cambios en el espectro de absorción (Figura $14 \mathrm{IVg}$ y Figura $14 \mathrm{IVh}$ ) no estén asociados a una degradación propiamente dicha de la curcumina sino a un rearreglo estructural de la $\beta-C D$ en disolución acuosa tal como lo reporta Alva et. al. [40].

Comparando los resultados mostrados en la Figura 14 y considerando la Ecuación 1 (Ver Capítulo 1), se calcula y se grafica el porcentaje de degradación de la 
curcumina como una función del tiempo bajo la influencia de $\beta$-CD (Figura 15). Se aprecia que en un medio amortiguado de $\mathrm{p} \beta-\mathrm{CD}=2$, bajo condiciones controladas, el porcentaje de degradación no es mayor al $3 \%$. Así mismo, se puede apreciar que en los primeros 20 minutos la respuesta es prácticamente líneal en todos los casos lo que permite calcular de estas líneas rectas la pendiente, la cual es proporcional a la $V_{\text {deg. }}$.
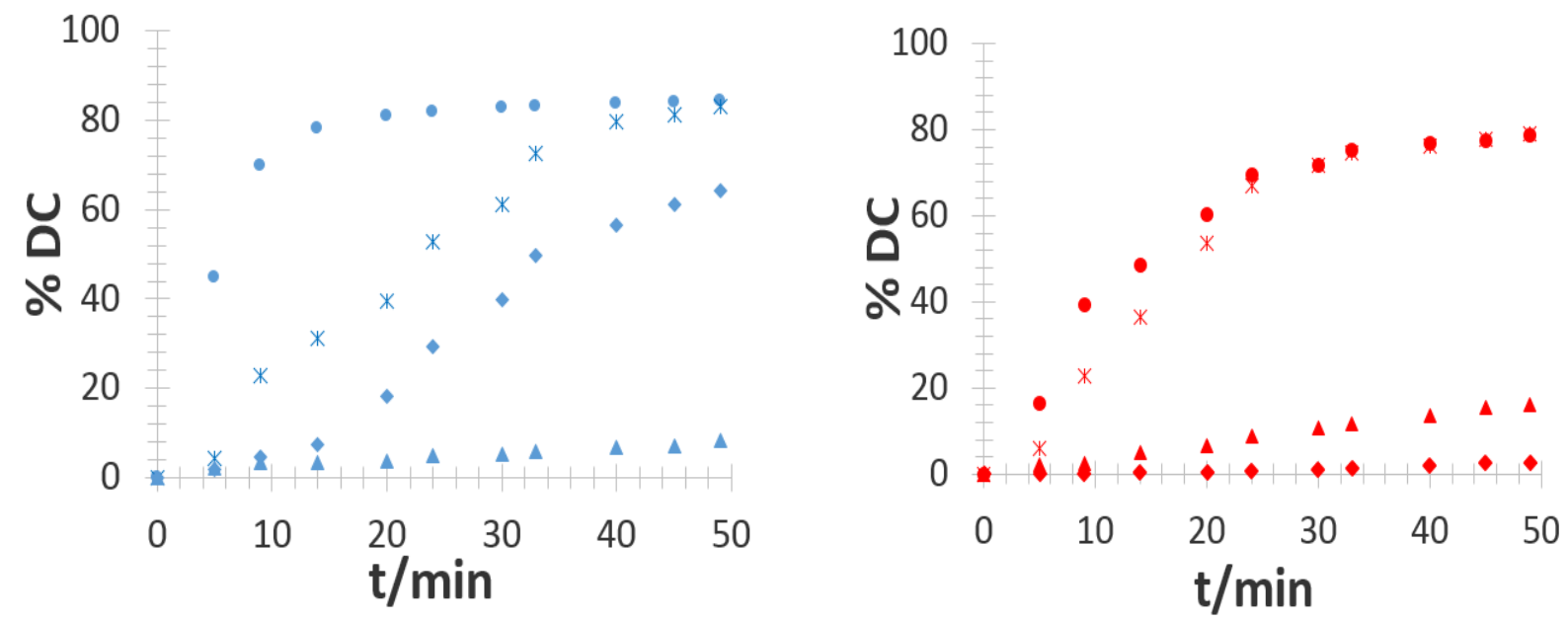

Figura 15. Porcentaje de degradación de la curcumina a pH $3.576 \pm 0.001$ a diferentes concentraciones de $\beta-C D(\bullet 0.0 \mathrm{mM}, * 0.6 \mathrm{mM}, 10.0 \mathrm{mM}$ y $\boldsymbol{\Delta} 16.6 \mathrm{mM})$. (Izquierda) sin control experimental, (Derecha) control de las condiciones experimentales.

A continuación, en la Tabla 7 se resumen los valores de la velocidad de degradación de la curcumina siguiendo el mismo tratamiento de datos que en el Capítulo 1. En todos los casos, la velocidad de degradación se ve disminuida cuando son controladas las condiciones experimentales. Considerando los resultados mostrados en la Figura 14 y Figura 15 así como los datos de la Tabla 7 se considerará que la concentración de $\beta-C D$ óptima para garantizar la estabilidad de la curcumina en disolución acuosa es de $10.0 \mathrm{mM}(\mathrm{p} \beta-\mathrm{CD}=2)$.

Tabla 7. Valores de velocidad de degradación $\left(\mathrm{V}_{\text {deg }}\right)$ de una disolución acuosa de curcumina ( $\mathrm{pH} 3.576 \pm 0.001$ ) a diferentes concentraciones de $\beta-C D$ y condiciones experimentales.

\begin{tabular}{|c|cc|}
\hline $\begin{array}{c}\text { Concentración de } \boldsymbol{\beta}-\mathrm{CD} \\
{[\mathrm{mM}]}\end{array}$ & Condiciones No controladas & Condiciones controladas \\
\hline $\mathbf{0 . 0}$ & $7.80 \pm 0.75$ & $2.86 \pm 0.29$ \\
\hline $\mathbf{0 . 6}$ & $2.48 \pm 1.08$ & $2.46 \pm 0.86$ \\
\hline $\mathbf{1 0 . 0}$ & $0.52 \pm 0.09$ & $0.02 \pm 0.01$ \\
\hline $\mathbf{1 6 . 6}$ & $0.40 \pm 0.03$ & $0.30 \pm 0.11$ \\
\hline
\end{tabular}


3.2.5 Estudio de la influencia de las condiciones experimentales sobre la estabilidad de la curcumina en medio acuoso por voltamperometría cíclica en medio amortiguado de $p \beta-C D=2$.

Para confirmar la estabilidad a $p \beta-C D=2$, se propone hacer un estudio de estabilidad por voltamperometría cíclica de la curcumina en medio acuoso en presencia de $10.0 \mathrm{mM}$ de $\beta-\mathrm{CD}$ a pH $3.576 \pm 0.001$ sin control y bajo condiciones controladas.

De acuerdo a la Figura 16 cuando se tiene un valor de $p \beta-C D=2$ en disolución bajo condiciones no controladas (Izquierda) se aprecia que el primer pico de oxidación, $\mathrm{Epa}_{\mathrm{l}}$, localizado a $0.778 \mathrm{~V}$ presenta una caída en la respuesta de corriente del $22.5 \%$ en 50 minutos (ver inserto). Cuando se tienen condiciones controladas

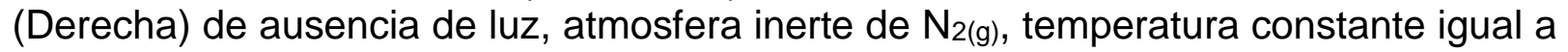
$(21.0 \pm 0.1)^{\circ} \mathrm{C}$ (ver inserto) la caída en la respuesta de corriente a $0.778 \mathrm{~V}$ es del 19.7 $\%$. En ambos casos, el proceso de oxidación a $\mathrm{Ep}_{\mathrm{a}}{ }^{0.670 \mathrm{~V}}$ que está presente cuando no se tiene $\beta$-CD desaparece (ver Figura 5). Probablemente esto sea a causa de que la $\beta$-CD está protegiendo los sitios de oxidación (los grupos -OH) de la curcumina. Esto demuestra la habilidad de la $\beta$-CD para proteger grupos fácilmente oxidables de la curcumina y por tanto asegurar la integridad de la curcumina.
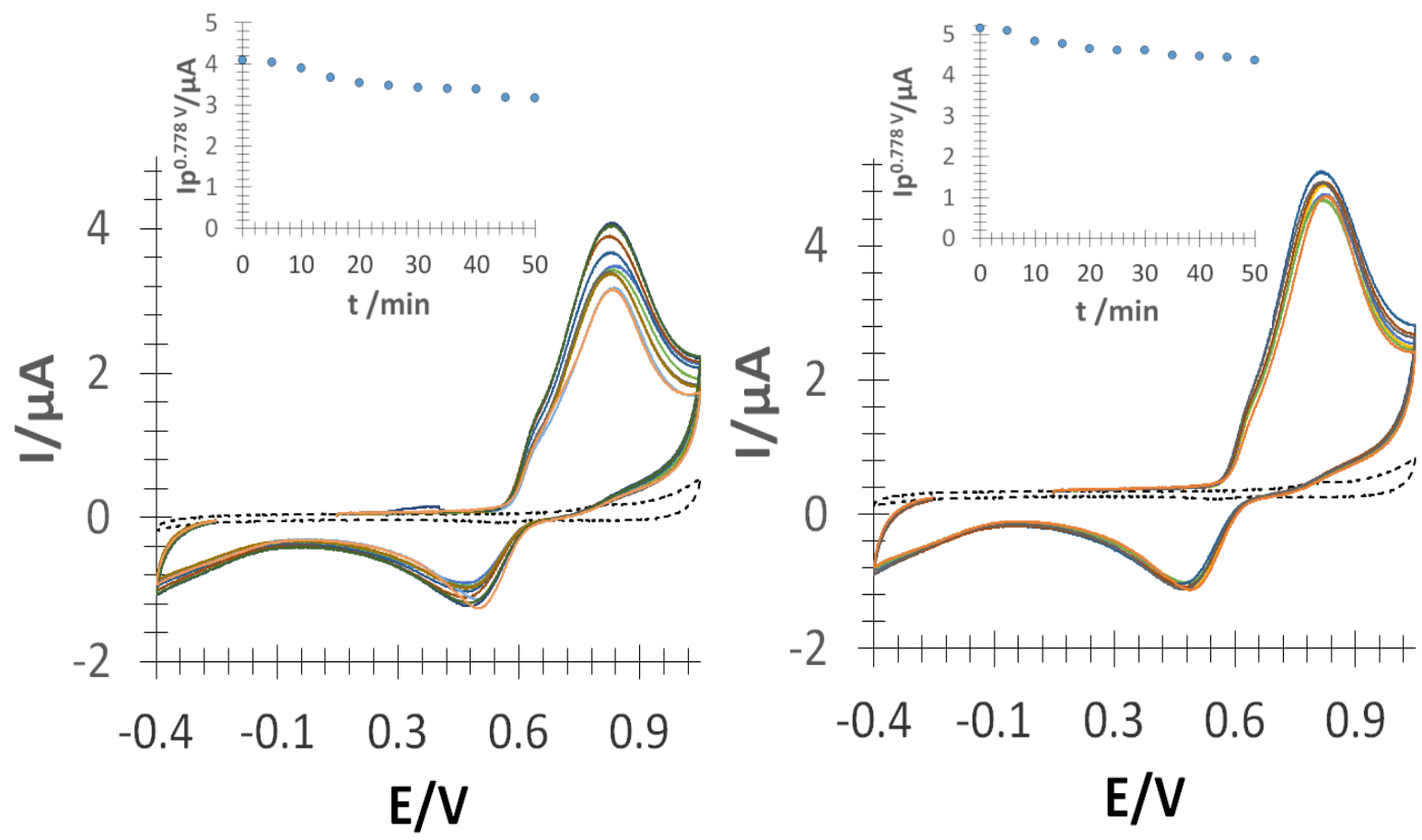

Figura 16. Voltamperogramas cíclicos de la curcumina en medio acuoso a pH $3.576 \pm 0.001$ a $\mathrm{p} \beta-\mathrm{CD}=2$. (Izquierda) $\sin$ control y (derecha) bajo condiciones controladas. El registro punteado negro es el blanco. El inserto muestra la variación temporal de la $\mathrm{Ipa}_{\mathrm{a}}^{0.778 \mathrm{~V}}$. 
A continuación, en la Tabla 8 se resumen los valores de $V_{\text {deg }}$ de la curcumina en ausencia de $\beta-C D$ y a un valor de $p \beta-C D=2$ por espectrofotometría de UV-Vis bajo condiciones no controladas y controladas a pH $3.576 \pm 0.001$. En la Tabla 9 se resumen los mismos valores calculados por voltamperometría cíclica.

Tabla 8. Valores de velocidad de degradación $\left(\mathrm{V}_{\text {deg }} / \% \mathrm{DC} \mathrm{min}^{-1}\right)$ de una disolución acuosa de curcumina en ausencia de $\beta-C D$ y a $p-\beta-C D=2$ por espectrofotometría de UV-Vis a $\mathrm{pH} 3.576 \pm 0.001$.

\begin{tabular}{|c|c|}
\hline \multicolumn{2}{|c|}{$\operatorname{Sin} \beta-C D$} \\
\hline No control & Control \\
\hline $7.80 \pm 0.75$ & $2.86 \pm 0.29$ \\
\hline \multicolumn{2}{|c|}{$p \beta-C D=2$} \\
\hline No control & Control \\
\hline $0.52 \pm 0.09$ & $0.02 \pm 0.01$ \\
\hline
\end{tabular}

Tabla 9. Valores de velocidad de degradación $\left(\mathrm{V}_{\mathrm{deg}} / \% \mathrm{DC} \min ^{-1}\right)$ de una disolución acuosa de curcumina en ausencia de $\beta-C D$ y a $p-\beta-C D=2$ por voltamperometría cíclica a pH $3.576 \pm 0.001$.

\begin{tabular}{|c|c|}
\hline \multicolumn{2}{|c|}{$\operatorname{Sin} \beta-C D$} \\
\hline No control & Control \\
\hline $5.96 \pm 0.17$ & $4.85 \pm 0.54$ \\
\hline \multicolumn{2}{|c|}{$p \beta-C D=2$} \\
\hline No control & Control \\
\hline $0.73 \pm 0.08$ & $0.30 \pm 0.05$ \\
\hline
\end{tabular}

A partir de los resultados de la Tabla 8 y 9 , se demuestra que la $V_{\text {deg }}$ de la curcumina en medio acuoso disminuye 390 veces por espectrofotometría de UV-Vis y 20 veces por voltamperometría cíclica siempre y cuando exista un control de las condiciones experimentales como son: ausencia de luz, atmósfera inerte de $\mathrm{N}_{2}(\mathrm{~g})$, temperatura constante de $(21.0 \pm 0.1){ }^{\circ} \mathrm{C}$ y $\mathrm{p} \beta-\mathrm{CD}=2 \mathrm{a} \mathrm{pH} 3.576 \pm 0.001$. La comparación se hace con respecto a ningún control experimental. 


\subsection{Determinación por espectrofotometría de UV-Vis de la constante del complejo de inclusión Curcumina- $\beta-C D$.}

Tang et. al. [21] reportan la interacción entre la curcumina y la $\beta-C D$ en medio acuoso a pH ácido mediante un compuesto de inclusión Curcumina- $\beta-C D$, el cual reportan que es una interacción de 1 a 2 , una curcumina por dos $\beta$-CD cuyo valor de constante de inclusión que calculan es: $K_{\text {inclusión }}=5.53 \times 10^{5} \mathrm{~mol}^{-2} \mathrm{~L}^{2}$ a pH 2.4 y una temperatura de $(20 \pm 1)^{\circ} \mathrm{C}$. Sin embargo, en el trabajo de Tang et. al. no mencionan los cuidados considerados sobre las disoluciones acuosas de trabajo de curcumina.

De acuerdo a la evidencia mostrada en el punto 3.2 de que la $\beta$-CD promueve una mayor estabilidad de la curcumina en medio acuoso, se hace imperante conocer el valor de la constante del complejo de inclusión en medio acuoso para una mayor robustez en el diseño de las condiciones de análisis para el estudio de la curcumina en medio acuoso.

\subsubsection{Reactivos.}

Curcumina grado analítico marca Sigma Aldrich ( $\geq 99.5 \%), \beta$-ciclodextrina ( $\beta$ CD) marca Sigma Aldrich ( $\geq 97.0 \%$ ), lentejas de $\mathrm{NaOH}$ marca Macron, $\mathrm{HClO}_{4}$ concentrado.

\subsubsection{Equipo.}

La lectura de $\mathrm{pH}$ se realiza con un potenciómetro marca sensION equipado con un electrodo de vidrio de $\mathrm{Ag}^{\circ} \mid \mathrm{AgCl}[\mathrm{KCl}, 3 \mathrm{M}]$ marca Hach $5014 \mathrm{~T}$.

Para el control de la temperatura se utiliza un recirculador Grant W14.

Cada espectro de absorción se registra mediante un espectrofotómetro Lambda 20 UV Vis equipado con celdas de cuarzo $1 \mathrm{~cm}$ de longitud de paso óptico. El intervalo de adquisición de los espectros es de 200 a $700 \mathrm{~nm}$ a una velocidad de 1960 $\mathrm{nm}$ min $^{-1}$. 


\subsubsection{Método.}

Determinación de las constantes de inclusión del complejo curcumina- $\beta$ Ciclodextrina por UV Vis. Se preparan 13 disoluciones de concentración variable de $\beta$ $\mathrm{CD}$ (desde $0 \mathrm{mM}$ hasta $16.6 \mathrm{mM}$ ). La concentración de curcumina en el recipiente se mantiene a $[32.5 \mu \mathrm{M}]$ a pH $3.576 \pm 0.001$ para su lectura en el equipo. Para cada valor de concentración de $\beta-C D$ se utilizan disoluciones de curcumina recién preparadas bajo condiciones experimentales controladas.

\subsubsection{Cálculo de las constantes de inclusión de la curcumina- $\beta$-Ciclodextrina en medio ácido.}

En la Figura 17 se muestran los espectros de absorción de la curcumina en función de la concentración de $\beta$-CD. Existe un máximo de absorción a $423 \mathrm{~nm}$ el cual tiende a incrementarse conforme aumenta la concentración de $\beta$-CD en solución. Lo mismo ocurre con la banda a $357 \mathrm{~nm}$. El hecho de que la absorbancia incremente es debido a la rígidez que va adquiriendo la curcumina conforme se va incluyendo en la cavidad de la $\beta-C D$, es decir; su movimiento espacial se restringe.

A partir de un valor de $p \beta-C D=2$ desaparece la banda a $357 \mathrm{~nm}$ evidenciando una interacción gradual entre curcumina- $\beta-C D$ para formar el complejo de inclusión tal como se discutió en el punto 3.2.5. Donde la cavidad de la $\beta-C D$ bloquea las transiciones $\pi$ a $\pi^{*}$ de los dobles enlaces de la cadena alifática.

En el inserto de la Figura 17 se muestra el valor de absorbancia a $424 \mathrm{~nm}$ como una función del valor de $\mathrm{p} \beta-\mathrm{CD}$. Esta sigmoide muestra dos inflexiones importantes, una a $p \beta-C D=3.5$ y otra a $p \beta-C D=2$. Dado que en la celda se tiene curcumina y $\beta-C D$ se propone que estos puntos de inflexión están asociados a los valores de constante de inclusión. 


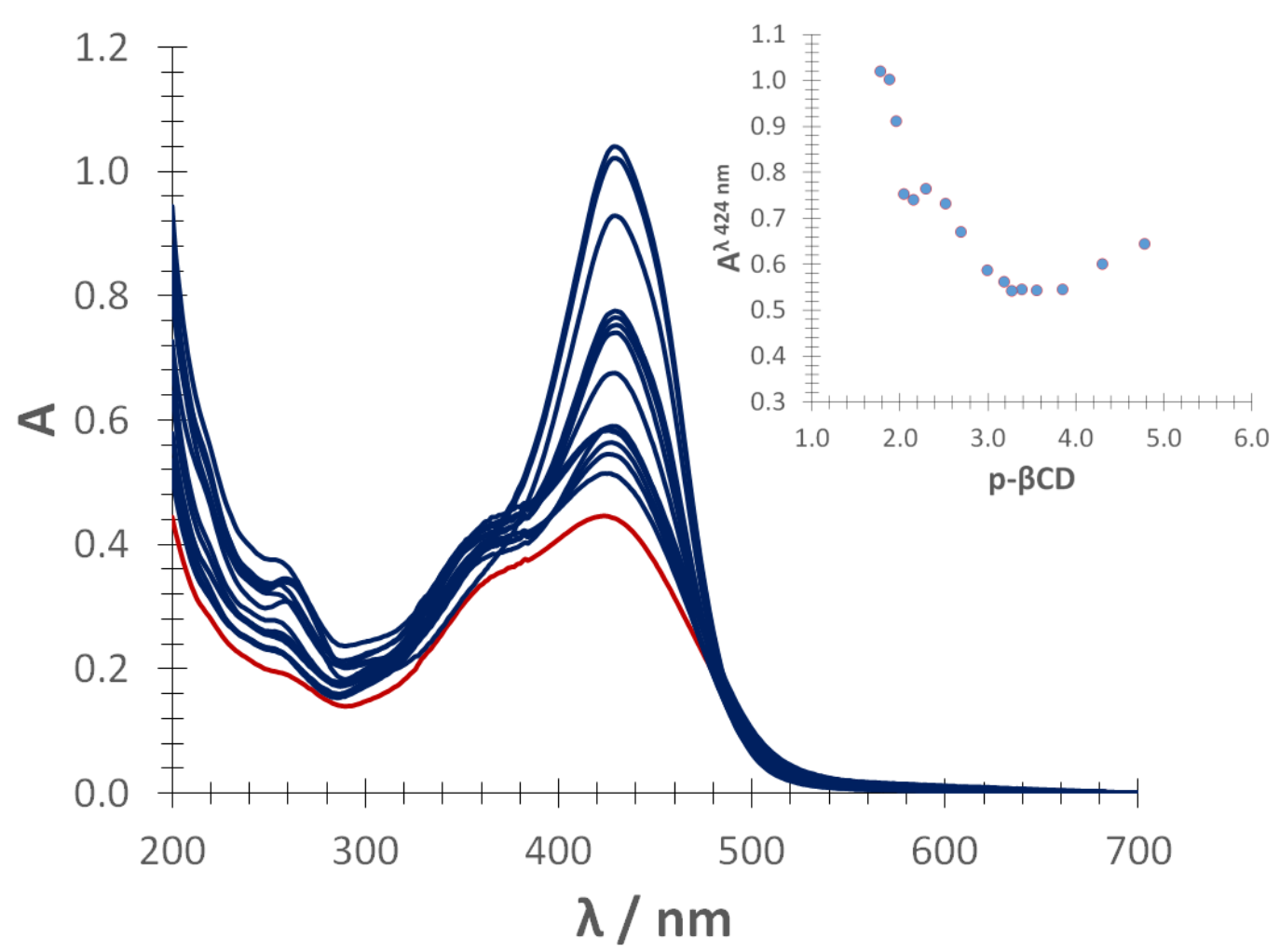

Figura 17. Espectros de absorción de curcumina R. A. ( $\geq 99.5 \%)[32.5 \mu \mathrm{M}]$ a pH $3.576 \pm$ 0.001 (registro rojo) a diferentes concentraciones de $\beta-C D$ (registros azules). En el inserto de figura se muestra la variación del máximo de absorbancia a $424 \mathrm{~nm}$ como una función de la concentración de $\beta-C D$ bajo condiciones controladas.

Toda vez que en el Capítulo 2 quedó demostrado la utilidad del software SQUAD para el cálculo y refinamiento de constantes en disolución acuosa; en este capítulo se propone nuevamente su uso para ajustar los datos experimentales y así encontrar los valores de log $\mathrm{K}$ que mejor describan el modelo de inclusión entre la curcumina y la $\beta$-CD. De esta manera, en la Tabla 10 se muestran los valores de las constantes de inclusión calculadas por el programa computacional SQUAD.

Tabla 10. Valores de las constantes sucesivas de inclusión refinadas por el programa computacional SQUAD para el sistema curcumina- $\beta$-CD usando 13 espectros de 4 $\mathrm{nm}$ en $4 \mathrm{~nm}$ cada uno a pH $3.576 \pm 0.001$ bajo condiciones experimentales controladas.

\begin{tabular}{|c|c|}
\hline \multirow{2}{*}{$\begin{array}{c}\mathrm{H}_{3} \mathrm{Cur}+\boldsymbol{\beta}-\mathrm{CD}=\mathrm{H}_{3} \mathrm{Cur} \beta-\mathrm{CD} \\
\mathrm{H}_{3} \mathrm{Cur} \beta-\mathrm{CD}+\boldsymbol{\beta}-\mathrm{CD}=\mathrm{H}_{3} \operatorname{Cur}(\beta-\mathrm{CD})_{2}\end{array}$} & $\log _{1} 3.889 \pm 0.065$ \\
\hline & $\log _{2} 2.139 \pm 0.050$ \\
\hline$\sigma$ & $1.468 \times 10^{-2}$ \\
\hline $\mathrm{U} / \boldsymbol{n}$ & $1.011 \times 10^{-4}$ \\
\hline
\end{tabular}


En la Figura 18 se muestran los coeficientes de extinción molar obtenidos por SQUAD con su respectiva desviación estándar.

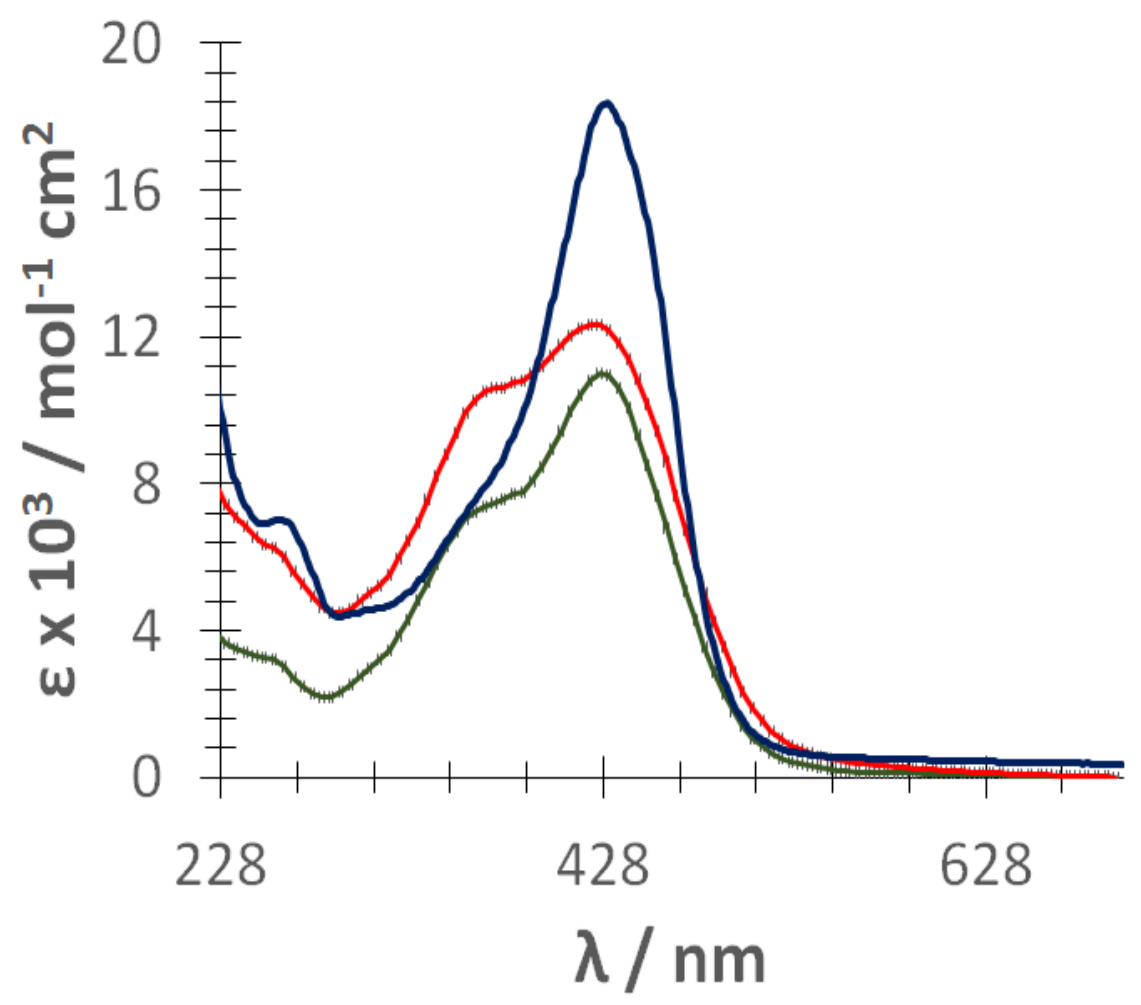

Figura 18. Coeficientes de extinción molar para la especie neutra de curcumina R. A. ( $\geq 99.5$ $\%)$ y las especies con $\beta-C D$ en función de la longitud de onda calculados por SQUAD. (-) H3Cur, (-) H3Cur( $\beta-C D),(-)$ H3Cur( $\beta-C D)_{2}$.

En la Figura 19 se muestra el ajuste de los espectros experimentales con los reconstruidos a partir de los coeficientes de extinción molar calculados por SQUAD. 


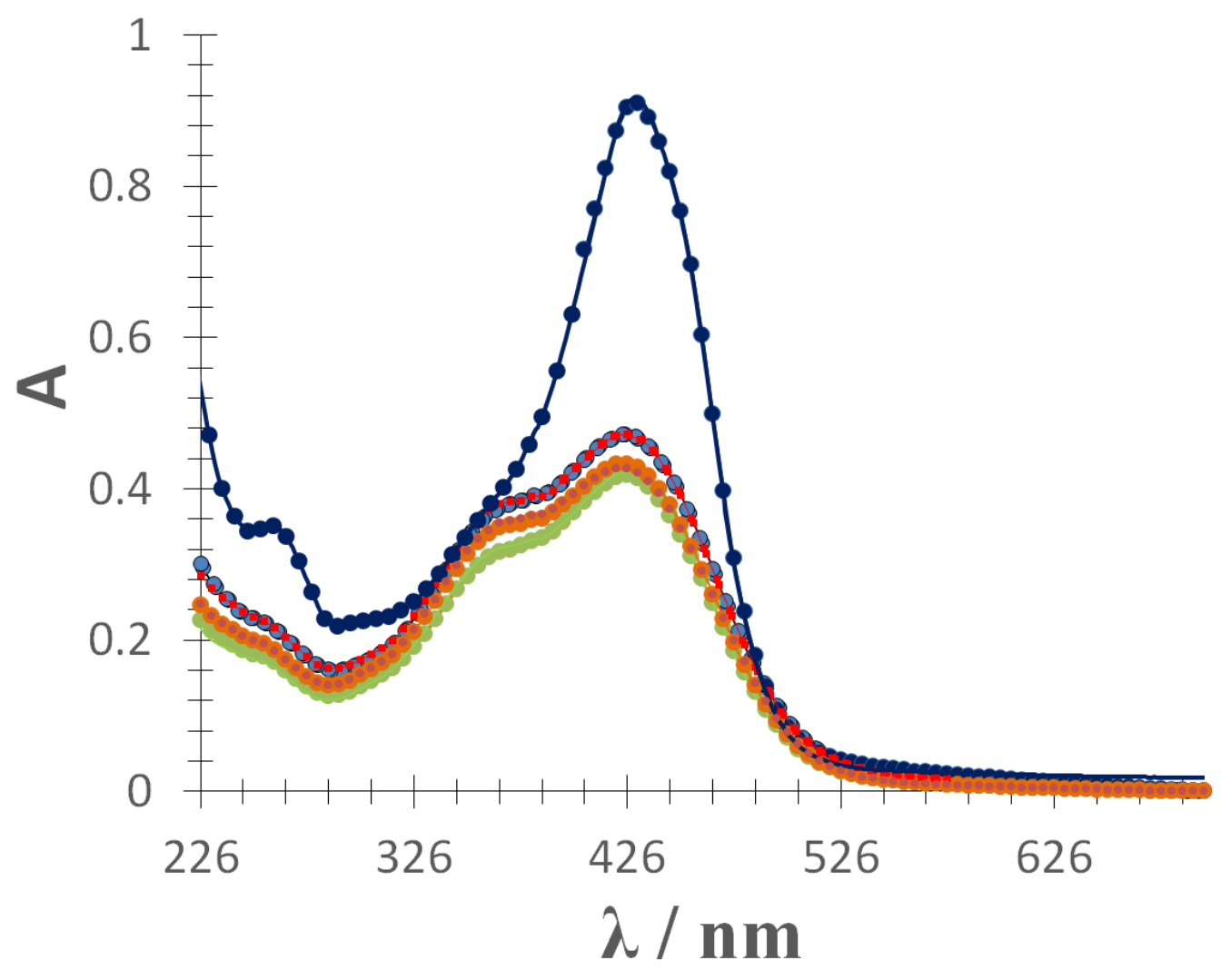

Figura 19. Ajuste de los espectros de absorción de la curcumina R. A. ( $\geq 99.5 \%$ ) en presencia de $\beta-C D$ a pH $3.576 \pm 0.001$. Los marcadores son los datos experimentales mientras que las líneas continuas son los espectros reconstruidos a partir de los coeficientes de extinción molar proporcionados por SQUAD. $(\bullet)[\beta-C D]=0,(\bullet) p \beta-C D=1.959,(\bullet) p \beta-C D$ $=3.852,(\bullet) \mathrm{p} \beta-\mathrm{CD}=4.308$.

Con esta información disponible es posible construir un diagrama de fracción molar de las especies en función del valor de $p \beta-C D$ (Figura 20). Los marcadores muestran el intervalo de $\mathrm{p} \beta-C D$ utilizado experimentalmente en este trabajo, abarcando así puntos por debajo y por arriba de cada valor de log $\mathrm{K}$ calculado. Es interesante destacar que el valor de log $\mathrm{K}$ obtenido por Tang y colaboradores es de 5.743 ( $\mathrm{pH} 2.4$ ) usando un valor de concentración máxima de $5 \mathrm{mM}$. Este valor, según el diagrama mostrado en la Figura 20 predice que no logran formar el complejo de curcumina con $\beta$-CD pues existe $98.62 \%$ de la especie $\mathrm{H}_{3} \mathrm{Cur}, 1.38 \%$ de la especie $\mathrm{H}_{3} \mathrm{Cur}(\beta-\mathrm{CD})$ y $0 \%$ de la especie $\mathrm{H}_{3} \mathrm{Cur}(\beta-C D)_{2}$. De esta manera se pone en evidencia la importancia del cuidado de las condiciones de trabajo sobre el estudio fisicoquímico de la curcumina en disolución acuosa y el impacto que tiene el software SQUAD para lograr un análisis correcto de los datos experimentales para el cálculo de las constantes. 
En el capítulo 3 punto 3.2 se propuso trabajar a una concentración de $10 \mathrm{mM}$ de $\beta-C D(p \beta-C D=2)$, esto quiere decir que bajo esas condiciones de concentración de $\beta-C D$ se tiene al menos dos complejos de inclusión siendo el $57.63 \%$ del complejo de inclusión $\mathrm{H}_{3} \mathrm{Cur}(\beta-\mathrm{CD})_{2}$ y $42.37 \%$ del complejo $\mathrm{H}_{3} \mathrm{Cur}(\beta-\mathrm{CD})$ en disolución acuosa a pH $3.576 \pm 0.001$.

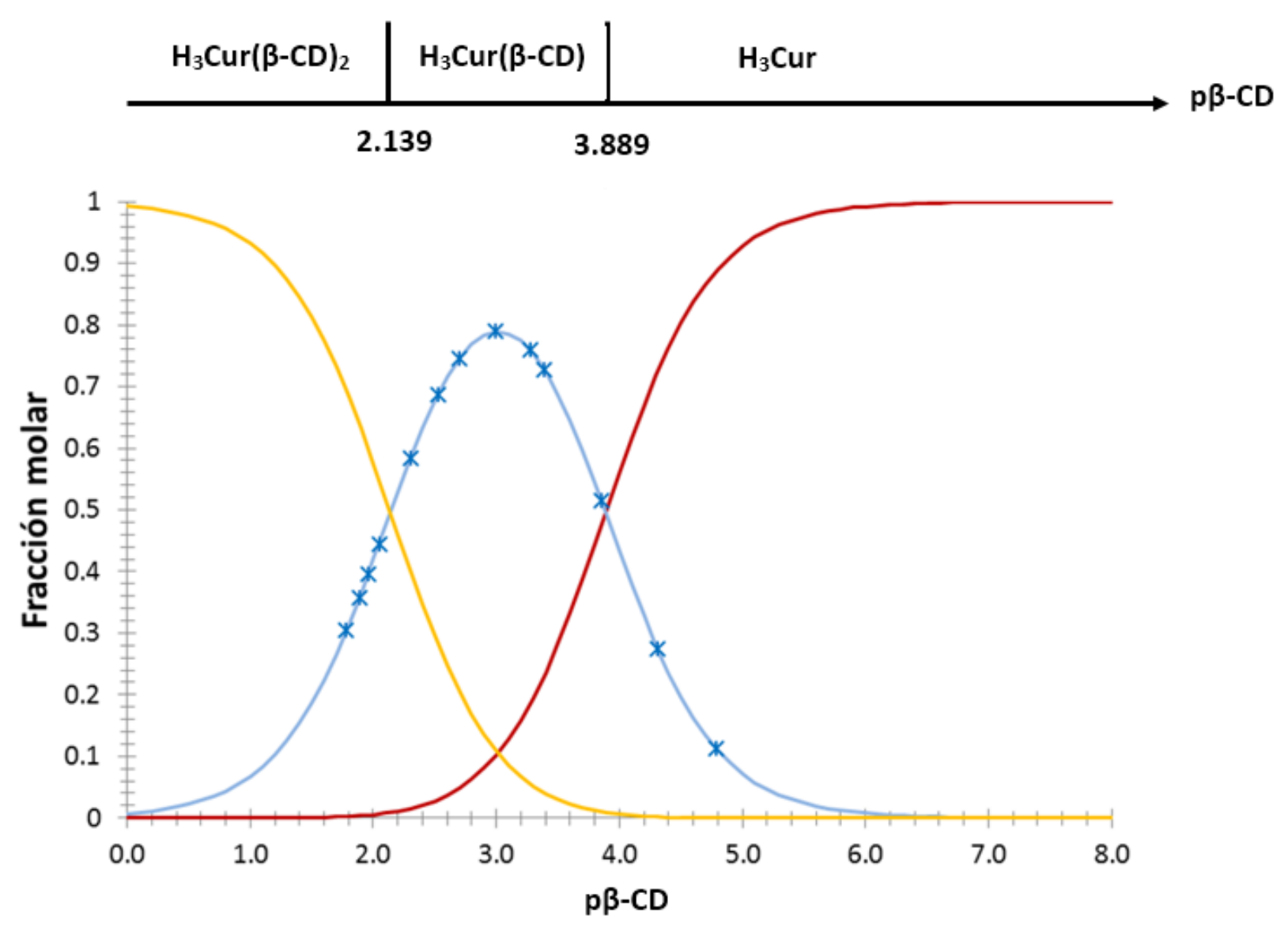

Figura 20. Diagrama de fracción molar de las especies de curcumina en función de $\mathrm{p} \beta C D$ a pH $3.576 \pm 0.001 ;(-) \mathrm{H}_{3} \mathrm{Cur}(\beta-C D)_{2},(-) \mathrm{H}_{3} \mathrm{Cur}(\beta-C D)$, y (-) $\mathrm{H}_{3}$ Cur.

\subsubsection{Cálculo de la constante de inclusión de la curcumina- $\beta$-Ciclodextrina en medio básico.}

No obstante que a pH ácidos se ha demostrado que la curcumina es inestable, y que su estabilidad aumenta conforme el valor de $\mathrm{pH}$ incrementa; se asume que los complejos entre curcumina y $\beta-C D$ también son estables bajo estas condiciones de $\mathrm{pH}$. Por tanto, se propone evaluar la constante de inclusión a pH básico. 
En la Figura 21 se muestran los espectros de absorción de la curcumina a diferentes concentraciones de $\beta-C D$ bajo condiciones controladas a pH $13.605 \pm$ 0.001 . Cabe destacar que para cada espectro de absorción se utilizó una disolución independiente (análisis puntual).

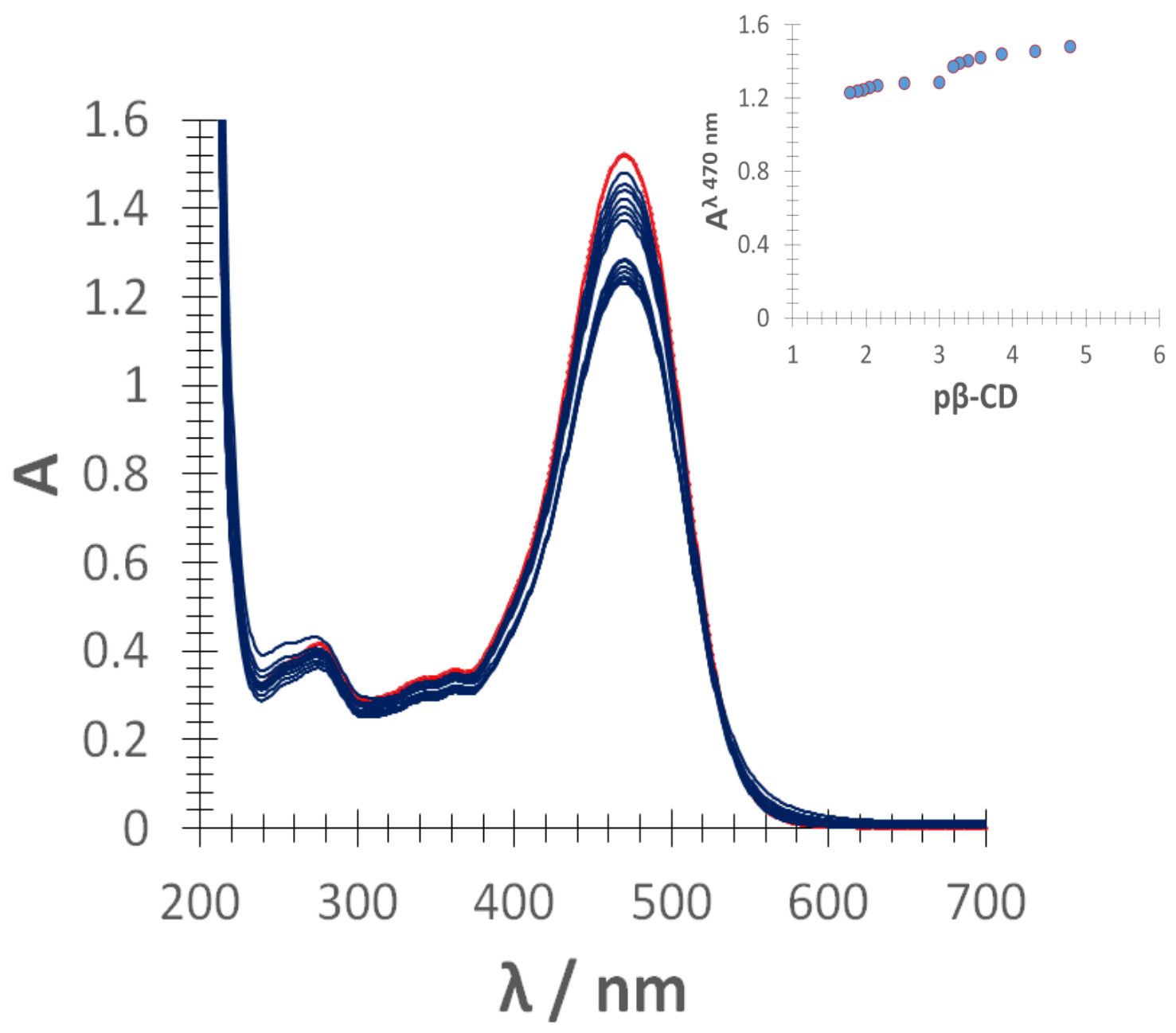

Figura 21. Espectros de absorción de curcumina R. A. ( $\geq 99.5 \%)[32.5 \mu \mathrm{M}]$ a pH $13.605 \pm$ 0.001 (registro rojo) a diferentes concentraciones de $\beta$-CD (registros azules). En el inserto de figura se muestra la variación del máximo de absorbancia a $470 \mathrm{~nm}$ como una función de $\mathrm{p} \beta$ $\mathrm{CD}$ bajo condiciones controladas.

De acuerdo a la Figura 21 se aprecia que conforme aumenta la concentración de $\beta-C D$ la respuesta de absorbancia disminuye (ver inserto de la Figura 21). Sin embargo, al hacer el análisis para determinar las constantes de inclusión con el software SQUAD, el cálculo no converge.

Es importante notar que a pH $13.605 \pm 0.001$, se favorece el predominio de la especie totalmente desprotonada de la curcumina. Tonnesen et. al. [41] reportan que la $\beta$-CD forma complejos de inclusión preferentemente donde la molécula huésped está especiada en su forma totalmente protonada (neutra) pudiendo así formar interacciones de Van der Waals y enlaces de tipo covalente necesarias para la 
estabilidad del complejo mismo. Es posible que por esta razón no se vea favorecido la formación total del complejo de inclusión en medio básico $(\mathrm{pH}>13)$ dada la ausencia de este tipo de interacciones químicas.

A continuación en el siguiente esquema se muestra el modelo que explica la interacción entre la curcumina, $\mathrm{H}_{3} \mathrm{Cur}$, y la $\beta-\mathrm{CD}$ a pH $3.576 \pm 0.001$.

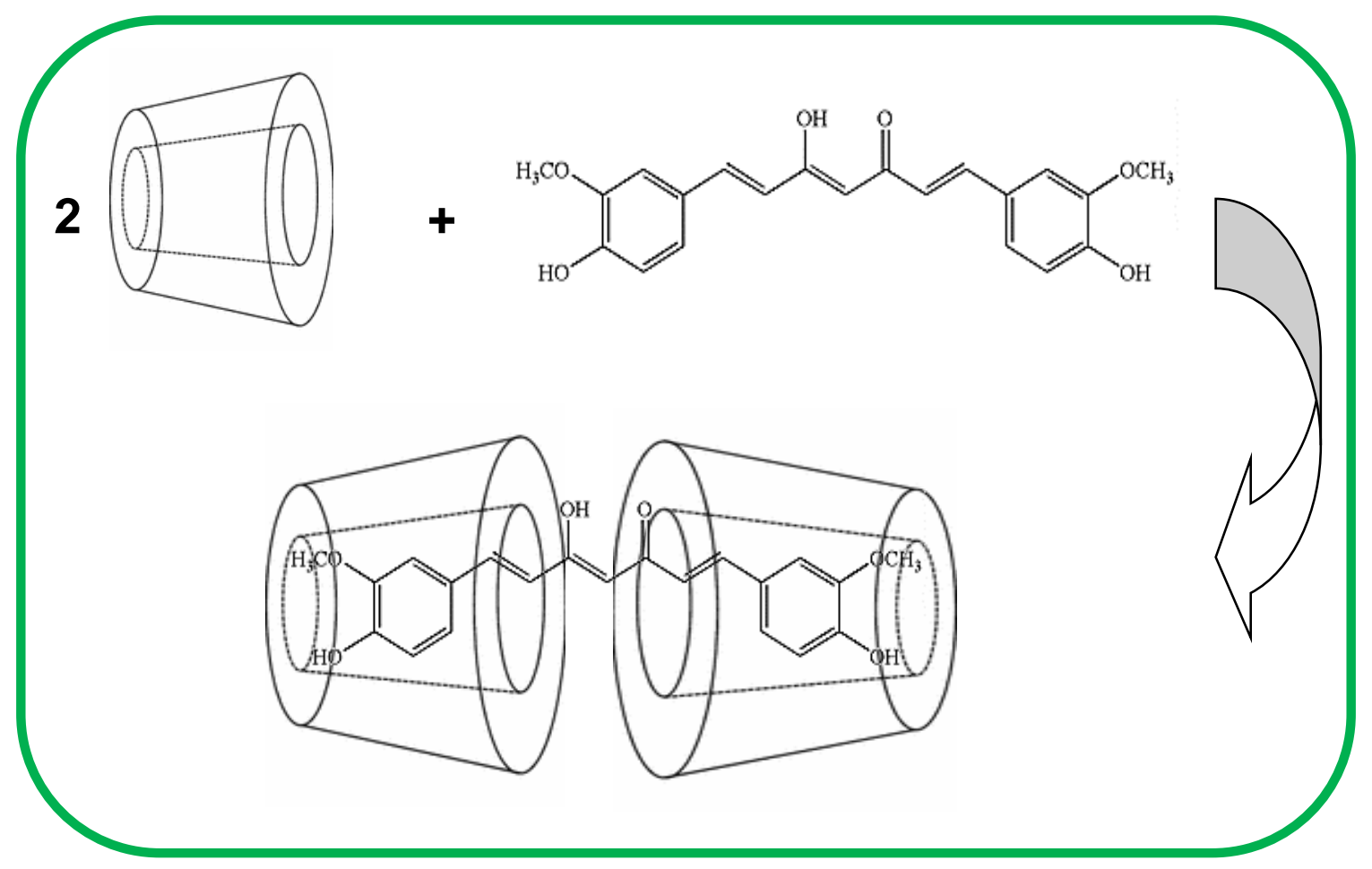

\subsection{Conclusiones.}

Se demostró que el porcentaje de degradación de la curcumina en medio ácido disminuye hasta 390 veces usando un monitoreo espectrofotométrico y 20 veces por voltamperometría cíclica amortiguando el medio a $\mathrm{p} \beta-C D=2(10 \mathrm{mM})$ siempre y cuando se trabaje bajo condiciones experimentales controladas.

El modelo que mejor explica la interacción entre la curcumina con la $\beta$-CD es mediante la formación de un complejo de inclusión "huésped-anfitrión". El refinamiento de las constantes con el software SQUAD permite calcular los siguientes valores: log $\mathrm{K}_{1}=3.889 \pm 0.065$ correspondiente al equilibrio químico: $\mathrm{H}_{3} \mathrm{Cur}+\beta-\mathrm{CD}=\mathrm{H}_{3} \mathrm{Cur}(\beta-$ CD) y $\log \mathrm{K}_{2}=2.139 \pm 0.050$ correspondiente al equilibrio químico $\mathrm{H}_{3} \mathrm{Cur}(\beta-\mathrm{CD})+\beta$ $\mathrm{CD}=\mathrm{H}_{3} \mathrm{Cur}(\beta-C D)_{2}$ además de obtener los valores de extinción molar para cada complejo de inclusión siendo el de estequiometría 1 a 2 el que más absorbe en el sistema. 


\section{CAPÍTULO 4. Método analítico para la cuantificación de Curcumina en disolución acuosa en presencia de $\beta$-Ciclodextrina.}

"Si he logrado ver más lejos ha sido porque he subido a hombros de gigantes"

- Isaac Newton 


\subsection{Antecedentes.}

Dada la importancia de la cúrcuma no solo en áreas como la medicina y farmacéutica sino como colorante en la industria textil y alimentaria se han desarrollado en los últimos años diferentes métodos analíticos para la cuantificación de curcumina y así buscar un criterio de control de calidad. En la Tabla 11 se muestran solo aquellos métodos analíticos representativos para la comparación de los resultados de este trabajo.

Tal como se aprecia en la Tabla 11 es común encontrar en la literatura que la cuantificación de curcumina se lleva a cabo en medios no acuosos dada su baja solubilidad y estabilidad en agua. Así mismo, está reportado que el uso de técnicas analíticas como Cromatografía de Líquidos de Alta Resolución (HPLC, por sus siglas en inglés) [44-46] puede ser una alternativa para encontrar mejores valores de límites de detección y cuantificación. Sin embargo, estos trabajos también están reportados en medios no acuosos como acetonitrilo, etanol, tetrahidrofurano, etc. Adicionalmente, un inconveniente en el uso de HPLC es que involucra grandes cantidades de fase móvil, lo cual hace de esta técnica poco amigable para el medio ambiente.

Tabla 11. Parámetros analíticos asociados a la cuantificación de curcumina reportados en la literatura.

$\begin{array}{cccccc}\begin{array}{c}\text { Método/condiciones } \\ \text { de trabajo }\end{array} & \text { Sensibilidad } & \begin{array}{c}\text { Límite de } \\ \text { detección }\end{array} & \begin{array}{c}\text { Límite de } \\ \text { cuantificación }\end{array} & \begin{array}{c}\text { Intervalo lineal } \\ \text { Ref. }\end{array} & \begin{array}{l}\text { Espectrofotometría de } \\ \text { UV Vis con }\end{array} \\ \begin{array}{c}\text { nanopartículas de } \\ \text { ácido poli L-Láctico }\end{array} & 0.0203 \pm 0.0004 & 0.13 \pm \mathrm{N} \text {. R. } & 0.27 \pm \mathrm{N} \text {. R. } & (10.85-108.60) & \begin{array}{c}\text { Buzane } \\ \text { Medio } \mathbf{C H}_{2} \mathbf{C l}_{2}: \mathbf{C H} \mathbf{O H}\end{array} \\ \mathrm{uA} \mu \mathrm{M}^{-1} & \mu \mathrm{M} & \mu \mathrm{M} & \mu \mathrm{M} & \text { llo }\end{array}$

$(1: 1)$

Espectrofotometría de

UV-Vis.

Medio: Acuoso,

[ClO4 $\left.4^{-}\right]$0.068 M,

Fuerza iónica: $0.56 \mathrm{M}$

pH $3.576 \pm 0.001$

$0.0200 \pm 0.0043$

$0.9973 \pm 0.0237$

$3.3244 \pm 0.0788$

$(3.4-27.0) \mu M$

Este

p $\beta$-CD 2

$\mathbf{u A} \boldsymbol{\mu} \mathbf{M}^{-1}$

$\mu \mathrm{M}$

$\mu \mathrm{M}$

trabajo

Ausencia de luz, atmósfera de $\mathrm{N}_{2(\mathrm{~g})}$, $\mathrm{T}=(21.0 \pm 0.1){ }^{\circ} \mathrm{C}$

Análisis puntual 
Voltamperometría cíclica

ET: Electrodo de carbón vítreo Medio: $\mathrm{CH}_{3} \mathrm{OH}$, N. R. $4.1 \pm N . R$.

N. R.

$(9.9-107)$

Ziyatdi

$\mu \mathrm{M}$ $\mu \mathrm{M}$ nova $0.1 \mathrm{M} \mathrm{LiClO}_{4}$

et.al

(2012)

Voltamperometría cíclica.

ET: EPC

Medio: Acuoso, [ClO4 $\left.4^{-}\right]$ $0.068 \mathrm{M}$,

Fuerza iónica: $0.56 \mathrm{M}$ $0.0914 \pm 0.007$ $2.5779 \pm 0.2186$

$8.5932 \pm 0.7288$

$\boldsymbol{\mu A} \boldsymbol{\mu} \mathbf{M}^{-1}$

$\boldsymbol{\mu M}$

$\boldsymbol{\mu M}$

(8.6- 27.0)

Este

pH $3.576 \pm 0.001$ p $\beta$-CD 2

Ausencia de luz, atmósfera de $\mathrm{N}_{2(\mathrm{~g})}$, $\mathbf{T}=(21.0 \pm 0.1){ }^{\circ} \mathrm{C}$

Análisis puntual N.R. No reportado.

\subsection{Diseño de un método analítico para la cuantificación de curcumina en medio amortiguado de $\beta-C D$ por espectrofotometría de UV Visible.}

En el capítulo 3 se demuestra la viabilidad para estabilizar a la curcumina en medio acuoso trabajando en medio amortiguado de $\mathrm{p} \beta-\mathrm{CD}=2.0$ y $\mathrm{pH} 3.576 \pm 0.001$ bajo condiciones de ausencia de luz, atmósfera de $\mathrm{N}_{2(\mathrm{~g})}$ y temperatura constante (21.0 $\pm 0.1)^{\circ} \mathrm{C}$. De esta manera, se pretende en este capítulo hacer la propuesta de un método de cuantificación en medio acuoso utilizando dos técnicas instrumentales: espectrofotometría de UV-Vis y voltamperometría cíclica considerando el control experimental ya mencionado.

\subsubsection{Reactivos.}

Curcumina grado analítico marca Sigma Aldrich ( $\geq 99.5 \%)$ sin ningún tratamiento posterior de purificación, $\beta$-ciclodextrina $(\beta-C D)$ marca Sigma Aldrich $(\geq$ $97.0 \%$ ), lentejas de $\mathrm{NaOH}$ marca Macron, $\mathrm{HClO}_{4}$ concentrado.

\subsubsection{Equipo.}

La lectura de $\mathrm{pH}$ se realiza con un potenciómetro marca sensION equipado con un electrodo de vidrio de $\mathrm{Ag}^{\circ} \mid \mathrm{AgCl}[\mathrm{KCl}, 3 \mathrm{M}]$ marca Hach $5014 \mathrm{~T}$. 
Para el control de la temperatura se utiliza un recirculador Grant W14.

Cada espectro de absorción se registra mediante un espectrofotómetro Lambda 20 UV Vis equipado con celdas de cuarzo $1 \mathrm{~cm}$ de longitud de paso óptico. El intervalo de adquisición de los espectros es de 200 a $700 \mathrm{~nm}$ a una velocidad de 1960 $\mathrm{nm} \min ^{-1}$.

\subsubsection{Método.}

Se obtiene por triplicado la respuesta de absorbancia en función de la concentración creciente de curcumina (análisis puntual) a pH $3.576 \pm 0.001$ y $\mathrm{p} \beta-\mathrm{CD}=$ 2 bajo condiciones experimentales controladas que se describen en el Capítulo 1

Se utiliza un intervalo lineal de concentraciones de curcumina de 3.4 a $27.0 \mu \mathrm{M}$.

\subsubsection{Cálculo de los parámetros analíticos de desempeño del método de cuantificación.}

En la Figura 22, se muestran la familia de espectros de absorbancia obtenidos como una función creciente de la concentración de curcumina en disolución acuosa en medio amortiguado de $\mathrm{pH}$ y de $\beta$-CD. Se aprecia que el máximo de absorción a $430 \mathrm{~nm}$ es proporcional a la concentración de curcumina en disolución acuosa tal como se muestra en el inserto de cada figura. El procedimiento se hace por triplicado mostrando posteriormente las tres ecuaciones de línea recta respectivas. 


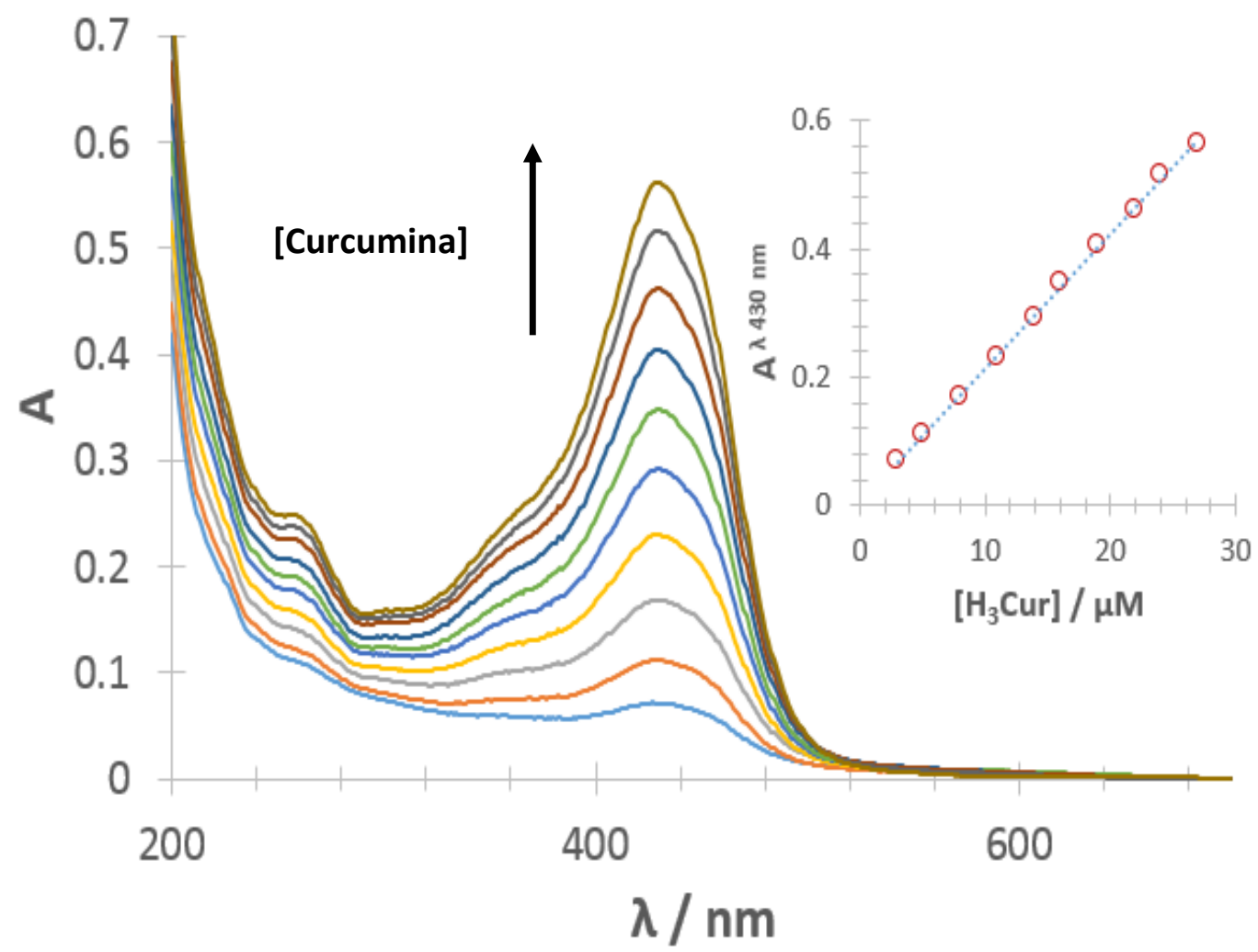

Figura 22. Familia de espectros de absorción en presencia de concentración creciente de curcumina $\left[\mathrm{H}_{3} \mathrm{Cur}\right]$ a $\mathrm{p} \beta-\mathrm{CD}=2.0$ y un valor de $\mathrm{pH} 3.576 \pm 0.001$ bajo condiciones experimentales controladas.

A continuación, se muestran las ecuaciones de línea recta asociadas a cada curva de calibración obtenida:

\section{Curva 1:}

$\mathrm{A}[\mathrm{uA}]=(0.0208 \pm 0.0003)\left[\mathrm{uA} \mu \mathrm{M}^{-1}\right]^{*}$ Curcumina $[\mu \mathrm{M}]+(0.0059 \pm 0.0044)[\mathrm{uA}]$ $r^{2}=0.999$

\section{Curva 2:}

$\mathrm{A}[\mathrm{uA}]=(0.0196 \pm 0.0003)\left[\mathrm{uA} \mu \mathrm{M}^{-1}\right]^{*}$ Curcumina $[\mu \mathrm{M}]+(0.0256 \pm 0.0046)[\mathrm{uA}]$ $r^{2}=0.998$

Curva 3:

$\mathrm{A}[\mathrm{uA}]=(0.0207 \pm 0.0003)\left[\mathrm{uA} \mu \mathrm{M}^{-1}\right]^{*}$ Curcumina $[\mu \mathrm{M}]+(0.0208 \pm 0.0050)[\mathrm{uA}]$ $r^{2}=0.998$ 
En la Tabla 12 se muestran los parámetros asociados al desempeño analítico del método para cada curva de calibración construída:

Tabla 12. Parámetros analíticos asociados a la cuantificación de curcumina determinados por espectrofotometría de UV-Vis.

\begin{tabular}{|c|c|c|c|c|}
\hline & $\begin{array}{l}\text { Sensibilidad } \\
{\left[\mathrm{UA} \mu \mathrm{M}^{-1}\right]}\end{array}$ & $\begin{array}{c}\text { LD }\left(3 s_{y x} m^{-1}\right) \\
{[\mu M]}\end{array}$ & $\begin{array}{c}\mathbf{L Q}\left(\mathbf{1 0 s}_{\mathrm{yx}} \mathrm{m}^{-1}\right) \\
{[\mu \mathrm{M}]}\end{array}$ & $\begin{array}{c}\text { Intervalo lineal } \\
{[\mu \mathrm{M}]}\end{array}$ \\
\hline Curva 1 & $0.0208 \pm 0.0003$ & $0.9185 \pm 0.0115$ & $3.0617 \pm 0.0382$ & \\
\hline Curva 2 & $0.0196 \pm 0.0003$ & $1.0192 \pm 0.0142$ & $3.3973 \pm 0.0472$ & $3.4-27.0$ \\
\hline Curva 3 & $0.0207 \pm 0.0003$ & $1.0543 \pm 0.0151$ & $3.5143 \pm 0.0503$ & \\
\hline Promedio & $0.0200 \pm 0.0043$ & $0.9973 \pm 0.0237$ & $3.3244 \pm 0.0788$ & \\
\hline
\end{tabular}

Donde:

uA: unidades de Absorbancia

$\mu \mathrm{M}$ : unidades de concentración en micro molar

LD: Límite de Detección

LQ: Límite de cuantificación

Syx: Error relativo de la curva de calibración

$\mathrm{m}$ : pendiente de la regresión lineal

Finalmente se hizo la prueba de recobro para tres niveles de la curva de calibración: $10 \%, 50 \%$ y $100 \%$. En la Tabla 13 se resumen estos resultados:

Tabla 13. Porcentajes de recobro para tres niveles de la curva de calibración obtenidos por espectrofotometría de UV VIS

\begin{tabular}{|c|cc|} 
& $\begin{array}{c}\text { Concentración } \\
\text { de curcumina } \\
{[\mu \mathrm{M}]}\end{array}$ & Recobro [\%] \\
\hline Nivel $\mathbf{1 0} \%$ & 3 & 99.8 \\
\hline Nivel $\mathbf{5 0} \%$ & 14 & 101.7 \\
\hline Nivel $\mathbf{1 0 0} \%$ & 27 & 99.9 \\
\hline
\end{tabular}

Comparando los valores de sensibilidad reportados en este trabajo con respecto a Silva-Buzanello et. al. [42] se aprecia que la sensibilidad es la misma aunque los valores LD y LQ tienden a ser mayores en este trabajo. Esto es a causa de que Silva-Buzanello y colaboradores trabajan en medios no acuosos, donde la 
curcumina es estable y por tanto, la $\mathrm{V}_{\text {deg }}=0$; además utilizan nanopartículas de ácido poli L-Láctico para mejorar el desempeño de su método propuesto.

\subsection{Diseño de un método analítico para la cuantificación de curcumina en medio amortiguado de $\beta-C D$ por voltamperometría cíclica.}

A continuación se muestra la propuesta de trabajo para la validación de un método analítico de cuantificación de curcumina en medio acuoso por la técnica electroquímica de voltamperometría cíclica.

\subsubsection{Reactivos.}

Curcumina grado analítico marca Sigma Aldrich ( $\geq 99.5 \%)$ sin ningún proceso posterior de purificación, $\beta$-ciclodextrina $(\beta-C D)$ marca Sigma Aldrich $(\geq 97.0 \%)$, lentejas de $\mathrm{NaOH}$ marca Macron, $\mathrm{HClO}_{4}$ concentrado.

\subsubsection{Equipo.}

La lectura de $\mathrm{pH}$ se realiza con un potenciómetro marca sensION equipado con un electrodo de vidrio de $\mathrm{Ag}^{\circ} \mid \mathrm{AgCl}[\mathrm{KCl}, 3 \mathrm{M}]$ marca Hach $5014 \mathrm{~T}$.

Para el control de la temperatura se utiliza un recirculador Grant W14.

Se utiliza una celda convencional con capacidad para tres electrodos siendo el electrodo de pasta de carbono, el electrodo de trabajo preparado de acuerdo al método de Ramírez-Silva $[28,29]$. Se utiliza un electrodo de referencia de $\mathrm{Ag}^{\circ} \mid \mathrm{AgCl}$ $[\mathrm{KCl}, 3 \mathrm{M}]$ marca BAS MF-2052 y un alambre de platino BAS MW-1032 como contraelectrodo. Los registros voltamperométricos se hacen utilizando una ventana de potencial de $-350 \mathrm{mV}$ a $1000 \mathrm{mV}$, a una velocidad de barrido de $100 \mathrm{mV} \mathrm{s}^{-1}$ y considerando un ciclo de barrido hacia sentido anódico.

\subsubsection{Método.}

Se obtiene por triplicado la respuesta de corriente en función de la concentración creciente de curcumina bajo condiciones experimentales controladas en medio amortiguado de $\mathrm{p} \beta-\mathrm{CD}=2.0$ a pH $3.576 \pm$. 0.001 .

Se utiliza un intervalo lineal de concentraciones de curcumina de 8.6 a $27.0 \mu \mathrm{M}$. 


\subsubsection{Cálculo de los parámetros analíticos de desempeño del método de cuantificación.}

En la Figura 23, se muestran los registros de corriente en función del potencial obtenidos para una concentración creciente de curcumina en disolución acuosa en medio amortiguado de $\mathrm{pH}$ y de $\beta$-CD. Se aprecian dos picos, uno de oxidación y otro de reducción localizados a un $\mathrm{Ep}^{\mathrm{a}} 763 \mathrm{mV}$ y un $\mathrm{Ep}^{\mathrm{c}} 440 \mathrm{mV}$ respectivamente. Tras el análisis de la variación de corriente como una función de la concentración creciente de curcumina en disolución acuosa se encuentra que el pico de oxidación a $763 \mathrm{mV}$ es el que muestra una mejor tendencia lineal en todos los casos. El procedimiento se hace por triplicado y mostrando las ecuaciones de línea recta respectivas.

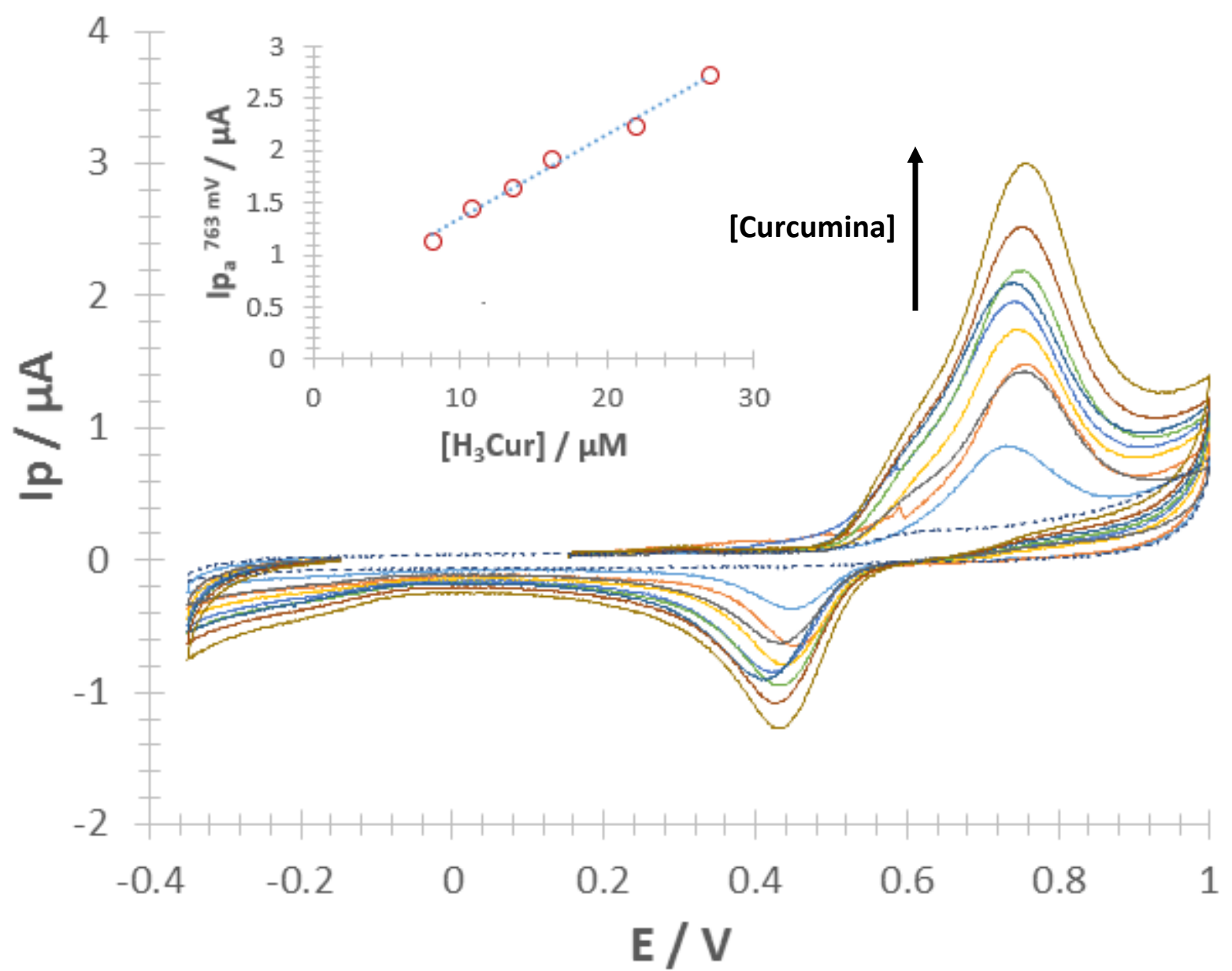

Figura 23. Voltamperogramas cíclicos en presencia de concentración creciente de curcumina [ $\mathrm{H}_{3} \mathrm{Cur}$ a $\mathrm{p} \beta-\mathrm{CD} 2.0$ y un valor de $\mathrm{pH} 3.576 \pm 0.001$ bajo condiciones experimentales controladas. ET: EPC, ER: $\mathrm{Ag}^{\circ} \mid \mathrm{AgCl}[\mathrm{KCl} 3 \mathrm{M}]$, EA: Alambre de platino. v $100 \mathrm{mV} \mathrm{s}^{-1}$, un ciclo de barrido.

A continuación, se muestran las ecuaciones de línea recta asociadas a cada curva de calibración: 
Curva 1:

$\mathrm{I} /[\mu \mathrm{A}]=(0.0799 \pm 0.0040)\left[\mu \mathrm{A} \mu \mathrm{M}^{-1}\right]^{*}$ Curcumina $[\mu \mathrm{M}]+(0.5507 \pm 0.0696)[\mu \mathrm{A}]$ $r=0.990$

\section{Curva 2:}

$\mathrm{I} /[\mu \mathrm{A}]=(0.0947 \pm 0.0045)\left[\mu \mathrm{A} \mu \mathrm{M}^{-1}\right] *$ Curcumina $[\mu \mathrm{M}]+(0.2103 \pm 0.0644)$ $[\mu \mathrm{A}]$

$r=0.991$

\section{Curva 3:}

$$
\begin{aligned}
& \mathrm{I} /[\mu \mathrm{A}]=(0.0996 \pm 0.0049)\left[\mu \mathrm{A} \mu \mathrm{M}^{-1}\right]^{*} \text { Curcumina }[\mu \mathrm{M}]+(0.3466 \pm 0.0792)[\mu \mathrm{A}] \\
& \mathrm{r}=0.988
\end{aligned}
$$

En la Tabla 14 se muestran los parámetros asociados al desempeño analítico del método para cada curva de calibración construída:

Tabla 14. Parámetros analíticos asociados a la cuantificación de curcumina

\begin{tabular}{|c|c|c|c|c|}
\hline & $\begin{array}{c}\text { Sensibilidad } \\
{\left[\mu \mathrm{A} \mu \mathrm{M}^{-1}\right]}\end{array}$ & $\begin{array}{c}\operatorname{LD}\left(3 s_{y x} m^{-1}\right) \\
{[\mu M]}\end{array}$ & $\begin{array}{c}L Q\left(10 S_{y x} m^{-1}\right) \\
{[\mu M]}\end{array}$ & $\begin{array}{c}\text { Intervalo lineal } \\
{[\mu \mathrm{M}]}\end{array}$ \\
\hline Curva 1 & $0.0799 \pm 0.0040$ & $2.3634 \pm 0.1175$ & $7.8780 \pm 0.3916$ & \\
\hline Curva 2 & $0.0947 \pm 0.0045$ & $2.4039 \pm 0.1133$ & $8.0129 \pm 0.3777$ & $8.6-27.0$ \\
\hline Curva 3 & $0.0996 \pm 0.0049$ & $2.9666 \pm 0.1454$ & $9.8888 \pm 0.4849$ & \\
\hline Promedio & $0.0914 \pm 0.0078$ & $2.5779 \pm 0.2186$ & $8.5932 \pm 0.7288$ & \\
\hline
\end{tabular}
determinados por voltamperometría cíclica.

Donde:

$\mu \mathrm{A}$ : unidades de corriente en micro ampere $\mu \mathrm{M}$ : unidades de concentración en micro molar

LD: Límite de Detección

LQ: Límite de cuantificación

Syx: Error relativo de la curva de calibración

$\mathrm{m}$ : pendiente de la regresión lineal 
Comparando los valores de LD y LQ obtenidos por voltamperometría cíclica (ver Tabla 11) calculados bajo condiciones experimentales controladas y amortiguadas de $\mathrm{p} \beta-\mathrm{CD}$ y $\mathrm{pH}$; es posible lograr valores de límite de detección 1.6 veces más abajo con respecto Ziyatdinova et. al. [43] haciendo de esta propuesta y condiciones analíticas competente con respecto a los métodos reportados en la literatura.

Así mismo, el método analítico propuesto en este capítulo por voltamperometría cíclica permite utilizar un electrodo de pasta de carbono como electrodo de trabajo lo cual hace de esta propuesta un método noble y de accessible costo, además de ser amigable con el medio ambiente por no usar disolventes no acuosos.

Es interesante comentar que por espectrofotometría de UV-Vis se registran procesos en disolución acuosa y en voltamperometría cíclica, por la naturaleza de la técnica, se registran procesos en la interfase conductor-disolución. Esto explica las diferencias en la sensibilidad entre ambas técnicas utilizadas.

El método analítico propuesto en este capítulo por espectrofotometría de UVVis y por voltamperometría cíclica es único con respecto a lo reportado en la literatura toda vez que es un método cuyas lecturas de respuesta de absorbancia como de corriente y potencial no presentan la influencia de manera significativa de la degradación de la curcumina en medio acuoso.

Finalmente se hizo la prueba de recobro para tres niveles de la curva de calibración: $10 \%, 50 \%$ y $100 \%$. En la Tabla 15 se resumen estos resultados:

Tabla 15. Porcentajes de recobro para tres niveles de la curva de calibración obtenidos por voltamperometría cíclica.

\begin{tabular}{|c|c|c|}
\hline & $\begin{array}{c}\text { Concentración } \\
\text { de curcumina } \\
{[\mu \mathrm{M}]}\end{array}$ & Recobro [\%] \\
\hline Nivel $10 \%$ & 3 & 98.5 \\
\hline Nivel $50 \%$ & 14 & 100.9 \\
\hline Nivel $100 \%$ & 27 & 98.9 \\
\hline
\end{tabular}




\subsection{Conclusiones}

Se implementaron las condiciones óptimas para el diseño de un método analítico para la cuantificación de curcumina en disolución acuosa tanto por espectrofotometría de UV-Vis como por voltamperometría cíclica en medio amortiguado de $\beta$-CD y de $\mathrm{pH}$ con valores de límite de detección y cuantificación que pueden competir con lo reportado en la literatura. El método de cuantificación propuesto en este trabajo ofrece límites de detección y cuantificación con el menor aporte en la $V_{\text {deg }}$ de la curcumina en medio ácido.

Esto permite abrir la posibilidad de llevar a cabo la cuantificación de curcumina sin la necesidad de utilizar disolventes orgánicos tóxicos tanto para el ser humano como para el medio ambiente o el uso de materiales sofisticados como electrodos de platino o de oro cuyo precio en el mercado es elevado.

Otra ventaja que representa esta propuesta de cuantificación con respecto a lo que ya está reportado en la literatura es que este método se lleva a cabo en una celda de trabajo con una capacidad de volumen no mayor a los $5.0 \mathrm{~mL}$ siendo así un método con una limitada generación de residuos. 


\section{CAPÍTULO 5. CONCLUSIONES GENERALES}

"Solo con el corazón se puede ver bien, lo esencial es invisible a los ojos"

- Antoine de Saint-Exupéry 


\subsection{Conclusiones generales.}

En el presente trabajo se demostró a tres diferentes valores de $\mathrm{pH} 3.576,7.025$ y 10.526 la influencia de la luz incidente, el $\mathrm{O}_{2(\mathrm{~g})}$ disuelto y temperatura en la estabilidad química de la curcumina en disolución acuosa. Se calculan los valores de $V_{\text {deg }}$ a cada valor de $\mathrm{pH}$ encontrando que esta disminuye cuando se trabaja bajo condiciones experimentales controladas las cuales se definen como aquellas donde se trabaja en ausencia de luz, atmósfera de $\mathrm{N}_{2(\mathrm{~g})}$ y temperatura constante de (21.0 \pm $0.1)^{\circ} \mathrm{C}$. En todos los casos estudiados la $\mathrm{V}_{\text {deg }}$ disminuye conforme el $\mathrm{pH}$ se hace más básico

Bajo condiciones experimentales controladas se determinaron tres constantes de acidez para la curcumina en medio acuoso: pka $1=7.428 \pm 0.015$ correspondiente al equilibrio $\mathrm{H}_{3} \mathrm{Cur}=\mathrm{H}_{2} \mathrm{Cur}^{-}+\mathrm{H}^{+}$, pka $=9.552 \pm 0.024$ correspondiente a la segunda disociación $\mathrm{H}_{2} \mathrm{Cur}^{-}=\mathrm{HCur}^{2-}+\mathrm{H}^{+}$y pka ${ }_{3}=10.946 \pm 0.034$ para la última disociación ácida $\mathrm{HCur}^{2-}=\mathrm{Cur}^{3-}+\mathrm{H}^{+}$. Se determinaron los valores de los coeficientes de absortividad molar para cada una de las especies que absorben en el sistema utilizando el software SQUAD lo cual permite conocer la especiación de la curcumina en disolución acuosa siendo así el ion curcuminato la especie que mayor absorbe.

Se determinó en medio ácido que el porcentaje de degradación de la curcumina disminuye hasta 390 veces usando un monitoreo espectrofotométrico y 20 veces por voltamperometría cíclica amortiguando el medio a $p \beta-C D=2(10 \mathrm{mM})$ siempre y cuando se trabaje bajo condiciones experimentales controladas. Esto hace a la $\beta$-CD un agente estabilizante óptimo para garantizar la integridad de la curcumina en medio acuoso. Se puso en evidencia que existe una interacción entre la curcumina con la $\beta$-CD mediante la formación de un complejo de inclusión "huésped-anfitrión". El refinamiento de las constantes con el software SQUAD permitió calcular los siguientes valores: $\log \mathrm{K}_{1}=3.889 \pm 0.065$ correspondiente al equilibrio químico: $\mathrm{H}_{3} \mathrm{Cur}+\beta-\mathrm{CD}=$ $\mathrm{H}_{3} \mathrm{Cur}(\beta-\mathrm{CD})$ y $\log \mathrm{K}_{2}=2.139 \pm 0.050$ correspondiente al equilibrio químico $\mathrm{H}_{3} \mathrm{Cur}(\beta$ $C D)+\beta-C D=H_{3} C u r(\beta-C D)_{2}$ además de obtener los valores de extinción molar para cada complejo de inclusión siendo el de estequiometría 1 a 2 el que más absorbe en el sistema.

Se implementaron las condiciones óptimas para el diseño de un método analítico para la cuantificación de curcumina en disolución acuosa tanto por espectrofotometría de UV-Vis como por voltamperometría cíclica en medio amortiguado de $\beta$-CD y de $\mathrm{pH}$ con valores de límite de detección y cuantificación que pueden competir con lo reportado en la literatura. El método de cuantificación propuesto en este trabajo ofrece límites de detección y cuantificación con el menor aporte en la $V_{\text {deg }}$ de la curcumina en medio ácido. 
CAPÍTULO 6. PERSPECTIVAS

“Cuanto más atrás puedas mirar, más adelante verás" Winston Churchill 


\subsection{Perspectivas.}

$\checkmark$ Si bien la estrategia para estabilizar a la curcumina en medio acuoso no fue del todo exitosa mediante la formación de compuestos de coordinación con el Zn (II); se demostró que si existe una interacción entre la curcumina, $\mathrm{H}_{3} \mathrm{Cur}$, y el $\mathrm{Zn}$ (II). Por tanto, resulta conveniente explorar el estudio y determinar el valor de la constante de formación global, $\beta_{n}{ }^{\mathrm{Zn}(\mathrm{II}) / H 3 \mathrm{Cur}}$, del sistema coordinado $\mathrm{Zn}\left(\mathrm{H}_{3} \mathrm{Cur}\right) \mathrm{n}$.

$\checkmark \quad$ Se abre la ventana para explorar la interacción y la determinación de la constante de formación respectiva entre la curcumina y otros metales, por ejemplo: $\mathrm{Cu}$ (II) y Ni (II). Ampliando así el panorama para la propuesta de mejores estrategias para estabilizar, caracterizar y cuantificar a la curcumina en medio acuoso.

$\checkmark \quad$ Dado que se demuestra en este trabajo la posibilidad de cuantificar curcumina en disolución acuosa bajo condiciones experimentales controladas en medio amortiguado de $\beta-C D$ y $\mathrm{pH}$. Resulta provechoso explorar la selectividad del método analítico (interferencias) e incluso aplicar la cuantificación en muestras reales y por tanto, la incorporación de estrategias de extracción y/o limpieza de muestra eficientes.

$\checkmark \quad$ Si bien en este trabajo se demuestra que es posible estabilizar a la curcumina para el diseño de un método analítico de cuantificación en disolución. Resulta ventajoso ampliar la posible mejora en los valores de LD y LQ por voltamperometría cíclica mediante una modificación en la superficie del electrodo de trabajo polimerizando $\beta-C D$. 


\section{CAPÍTULO 7. REFERENCIAS}

“Cada lágrima enseña a los mortales una verdad"

- Platón 


\subsection{Referencias.}

[1] J. González-Albadalejo, D. Sanz, M. Claramunt, J. L. Lavandera, I. Alkorta, J. Elguero. Anales de la Real Academia de Farmacia 81 (2015) 278.

[2] R.J. Anto, G. Kuttan, K.V.D. Babu, K.N. Rajasekharan, R. Kuttan. Pharmacy and Pharmacology Communications 4 (1998) 103.

[3] A. Barik, B. Mishra, L. Shen, H. Mohan, R.M. Kadam, S. Dutta, H.Y. Zhang, K.I. Priyadarsini. Free Radical Biology \& Medicine 39 (2005) 811.

[4] N. Jha, S. Mishra, S.K. Jha, A. Surolia. Electrochimica Acta 151 (2015) 574.

[5] M.N.A. Rao. Journal of Pharmacy and Pharmacology 46 (1994) 1013.

[6] M.N.A. Rao. International Journal of Pharmaceutics 100 (1993) 93.

[7] M.K. Unnikrishnan, M.N.A. Rao. Pharmazie 50 (1995) 490.

[8] R.J. Anto, G. Kuttan, K.V.D. Babu, K.N. Rajasekharan, R. Kuttan. International Journal of Pharmaceutics 131 (1996) 1.

[9] H.H. Tonnesen, H. De Vries, J. Karlsen, G.B. Van Henegouwen. Journal of Pharmaceutical Sciences 76 (1987) 371.

[10] P. Cohly, S. Asad, S.K. Das, M.F. Angel, M. Rao. International Journal of Molecular Sciences $4(2003) 22$.

[11] S. Zhihua, R. Salto, J.Li, C. Craick. Bioorganic \& Medicinal Chemistry 1 (1993) 415.

[12] H.P.T. Ammon, M.A. Wahl. Planta Medicine 57 (1991) 1.

[13] Y.J. Wang, M.H. Pan, A.L. Cheng, L.I. Lin, Y.S. Ho, C.Y. Hsieh, J.K. Lin. Journal of Pharmaceutical and Biomedical Analysis 15 (1997) 1867.

[14] A. Galano, R.A. Diduk, M.T. Ramírez-Silva, G. Alarcón-Ángeles, A. RojasHernández. Chemical Physics 363 (2009) 13.

[15] F. Payton, P. Sandusky, W.L. Alworth. Journal of Natural Products 70 (2007) 143.

[16] H.H. Tonnesen, J. Karlsen. Erratum Z. Lebensm Unters Forsch A 180 (1985) 402.

[17] H.H. Tonnesen, J. Karlsen. Erratum Z. Lebensm Unters Forsch A 180 (1985) 132.

[18] Y.J. Wang, M.H. Pan, A.L. Cheng, Lin LI, Ho YS, Hsieh CY. Lin JK. Journal of Pharmaceutical and Biomedical Analysis 15 (1997) 1867.

[19] O.N. Gordon, C. Schneider. Trends in Molecular Medicine 18 (2012) 361.

[20] M. Bernabé-Pineda, M.T. Ramírez-Silva, M. Romero-Romo, E. González-Vergara y A. Rojas-Hernández. Spectrochimica Acta Part A 60 (2004) 1091.

[21] B. Tang, L. Ma, H-Y. Wang y G-Y. Zhang. Journal of Agricultural and Food Chemistry 50 (2002) 1355.

[22] K. Balasubramanian. Indian Journal of Chemistry 30 (1991) 61.

[23] Y. FaShun, S. Jian-Long, X. Wen-Hai, S. Liang, J. Hong-Fang. Nutrients 10 (2017) 1.

[24] F. Amanolahi, A. Mohammadi, R. Kazemi, H. Nassirli, B. Malaekeh. Avicenna Journal of Phytomedicine 7 (2017) 444.

[25] S. Corona-Avendaño, A. Rojas-Hernández, M.A. Romero-Romo. M. PalomarPardavé, M.T. Ramírez-Silva. Spectrochimica Acta A 61 (2005) 3139.

[26] R.A. Diduk, M.T. Ramírez- Silva, A. Galano, A. Merkoçi. The Journal of Physical Chemistry B 117 (2013) 12347. 
[27] B. Verastegui-Omaña, M. Palomar-Pardavé, A. Rojas-Hernández, S. CoronaAvendaño, M. Romero-Romo, M.T. Ramírez-Silva. Spectrochimica Acta Part A 143 (2015) 187.

[28] M.T. Ramírez-Silva, M.E. Palomar-Pardavé, I. González, A. Rojas-Hernández. Electroanalysis 7 (1995) 184.

[29] M.T. Ramírez-Silva, I. González. Electroanalysis 10 (1998) 336.

[30] S.V. Jovanovic, S. Steenken, C.W. Boone, M.G. Simic. Journal of the American Chemical Society 121 (1999) 9677.

[31] M. Bernabé-Pineda, M.T. Ramírez-Silva, M. Romero-Romo, E. González-Vergara y A. Rojas-Hernández. Spectrochimica Acta Part A 60 (2004) 1105.

[32] A. Barik, B. Mishra, L. Shen, H. Mohan, R.M. Kadam, S. Dutta, H.Y. Zhang, K.I. Priyadarsini, Free Radical Biology \& Medicine 39 (2005) 811.

[33] K. Krishnankutty, V.D. John. Synthesis and Reactivity in Inorganic and MetalOrganic Chemistry 33 (2003) 343.

[34] K. Krishnankutty, P. Venugopalan. Synthesis and Reactivity in Inorganic and Metal-Organic Chemistry 28 (1998) 1313.

[35] L. Baum, A. Ng. Journal of Alzheimer's Disease 6 (2004) 367.

[36] K.A. Connors. Chemical Reviews 97 (1997) 1325.

[37] L. Szente, J. Szejtli. Trends in Food Science and Technology 15 (2004) 137.

[38] G. Roa-Morales, L. Galicia, M.T. Ramírez-Silva. Journal of Inclusion Phenomena and Macrocyclic Chemistry 46 (2003) 139.

[39] G. Roa-Morales, L. Galicia, A. Rojas-Hernández, M.T. Ramírez-Silva. Electrochimica Acta 50 (2005) 1925.

[40] J.C. Alva-Ensastegui. Efecto de los surfactantes en la formación de complejos de inclusion. Tesis para obtener el grado de Doctor en Ciencias (Química). Universidad Autónoma Metropolitana Unidad Iztapalapa (2018) 88.

[41] H.H. Tonnesen, M. Masson y T. Loftsson. International Journal of Pharmaceutics 244 (2002) 127.

[42] R.A. da Silva-Buzanello, A. Caroline, E. Bona, L. Cardozo, P. Henrique. F. Vitória, y O. Hess. Food Chemistry 172 (2015) 99.

[43] G.K. Ziyatdinova, A.M. Nizamova, H.C. Budnikov. Journal of Analytical Chemistry 67 (2012) 591.

[44] R.M. Smith y B.A. Witowska. Analyst 109 (1984) 259.

[45] S.K. Chauhan, B.P. Singh y S. Agrawala. Short Communications Indian Journal of Pharmaceuticals Sciences (1999) 58.

[46] Y. Long, W. Zhang, F. Wang y Z. Chen. Journal of Pharmaceuticals Analysis 4 (2013) 325.

[47] S. Kallel Trabelsi, N. Belhadj Tahar, B. Trabelsi y R. Abdelhedi, Journal of Applied Electrochemistry; 35 (2005) 967.

[48] J. Kankare. Analytical Chemistry 42 (1970) 1322.

[49] R.J. Motekaitis y A.E. Martell. Canadian Journal of Chemistry 60 (1982) 168.

[50] A. Sabatini, A. Vacca y P. Gans. Talanta; 21 (1974) 45.

[51] D.J. Leggett y W.A.E. McBryde. Analytical Chemistry 47 (1975) 1065.

[52] A. Ringbom. Complexation in Analytical Chemistry, Wiley, New York, (1963) 348.

[53] D. Skoog, J. Holler. Principios de Análisis Instrumental. Cengage Learning (2008) $635,650$. 


\section{APÉNDICE 1}

\section{Estabilidad de la solución stock de curcumina [0.002 M] en medio acuoso.}

Considerando que las alícuotas que se tomaron para hacer las lecturas por espectrofotometría de UV-Vis y por voltamperometría cíclica para cada una de las pruebas efectuadas en este trabajo provienen de una disolución stock de curcumina en medio acuoso, preparada en $\mathrm{NaOH}[0.005 \mathrm{M}]$, hace necesario conocer la estabilidad de esta disolución stock la cual fue mantenida en refrigeración y fue

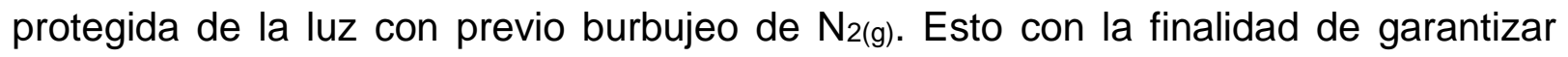
datos reproducibles y confiables.

En la Figura A1.1 se muestran los espectros de absorción de una disolución stock de curcumina $[0.002 \mathrm{M}]$ preparada en $\mathrm{NaOH}[0.005 \mathrm{M}]$ y mantenida en refrigeración $(4 \pm 1){ }^{\circ} \mathrm{C}$. El monitoreo de la respuesta de absorbancia fue registrada durante 4 horas con tomas de muestra independientes.

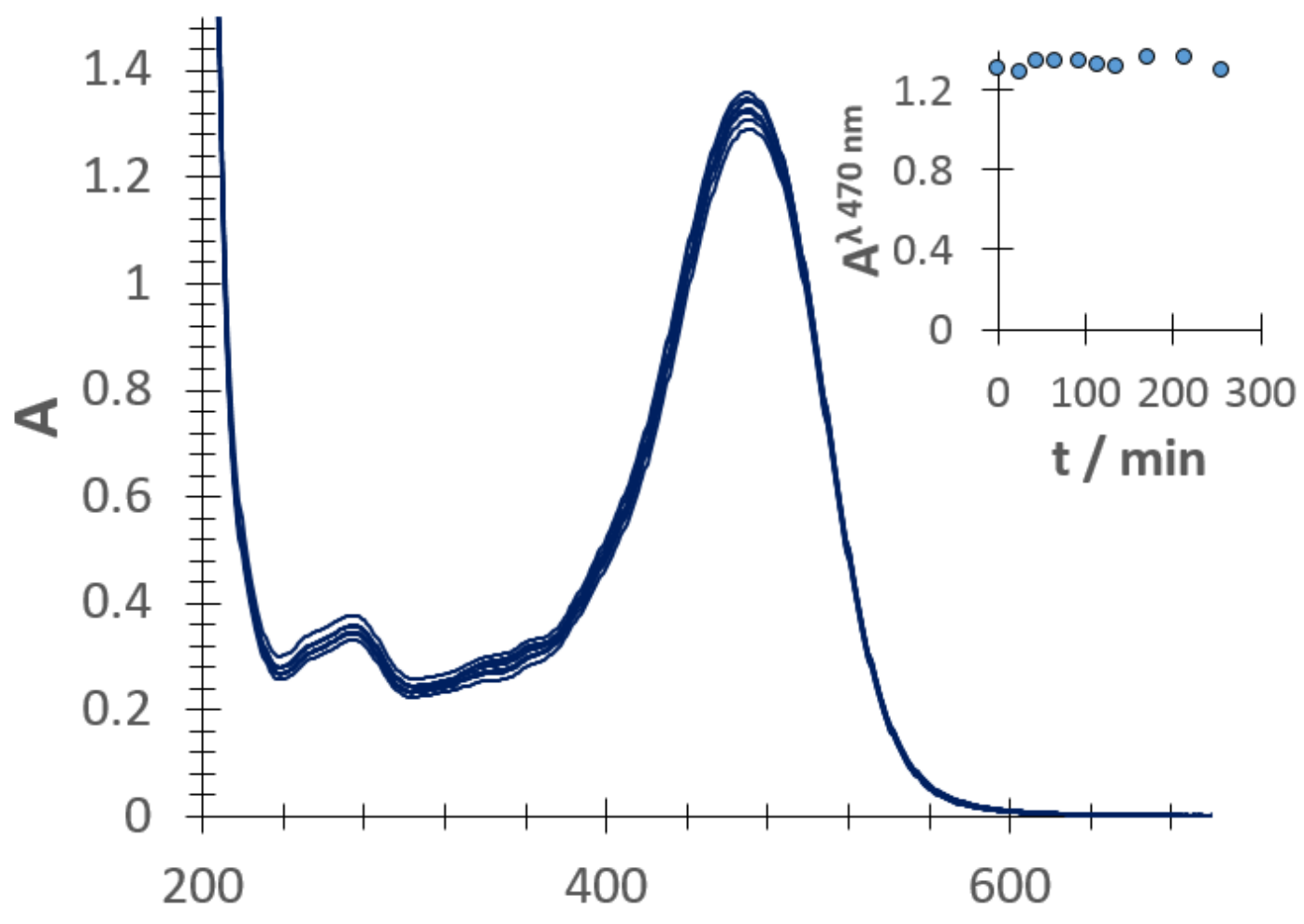

$\lambda / \mathrm{nm}$

Figura A1.1 Espectros de absorción de una disolución de curcumina [0.002 M] en $\mathrm{NaOH}$ $[0.005 \mathrm{M}]$ a $4{ }^{\circ} \mathrm{C}$ durante 4.26 horas a pH $11.698 \pm 0.001$. 
En la Figura A1.1 se muestra que el cambio en la respuesta de absorbancia después de transcurridos 4.2 horas fue del $1.04 \%$. Si bien esto nos garantiza que es posible trabajar con la misma disolución stock de curcumina durante cuatro horas; se tuvo la precaución de preparar una disolución stock de curcumina cada 60 minutos. Esto con la finalidad de disminuir al máximo posible la infuencia de la degradación química de la curcumina en medio acuoso. 


\section{APÉNDICE 2}

\section{Estudio $E_{\lambda}$ y propuesta del mecanismo de oxidación de la curcumina en medio acuoso.}

En la Figura A2.1 se muestra un estudio $E_{\lambda}$ de una disolución acuosa de curcumina $[32.5 \mu \mathrm{M}]$ a pH $3.576 \pm 0.001$ (bajo condiciones experimentales controladas) con el objetivo de esclarecer a que proceso de oxidación, si a $\mathrm{Ep}^{\mathrm{a}}=$ $0.670 \mathrm{~V}$ o Ep $\|^{\mathrm{a}}=0.813 \mathrm{~V}$, corresponde la reducción a $0.570 \mathrm{~V}$. Conforme se recorta la ventana de potencial hacia valores de potenciales más negativos el proceso de reducción $\mathrm{Epl}^{\mathrm{C}}=0.570 \mathrm{~V}$ tiende a desaparecer, es decir la corriente de pico catódico disminuye a la par que el proceso de oxidación $\mathrm{Ep}_{\|^{\mathrm{a}}}=0.813 \mathrm{~V}$ lo hace. Esto da evidencia de que la reducción es consecuencia del segundo proceso de oxidación. Por tanto, el primer proceso de oxidación, $\mathrm{Ep}^{\mathrm{a}}=0.670 \mathrm{~V}$ es irreversible mientras que el segundo $\mathrm{Ep}_{\mathrm{I}}^{\mathrm{a}}=0.813 \mathrm{~V}$ es reversible.

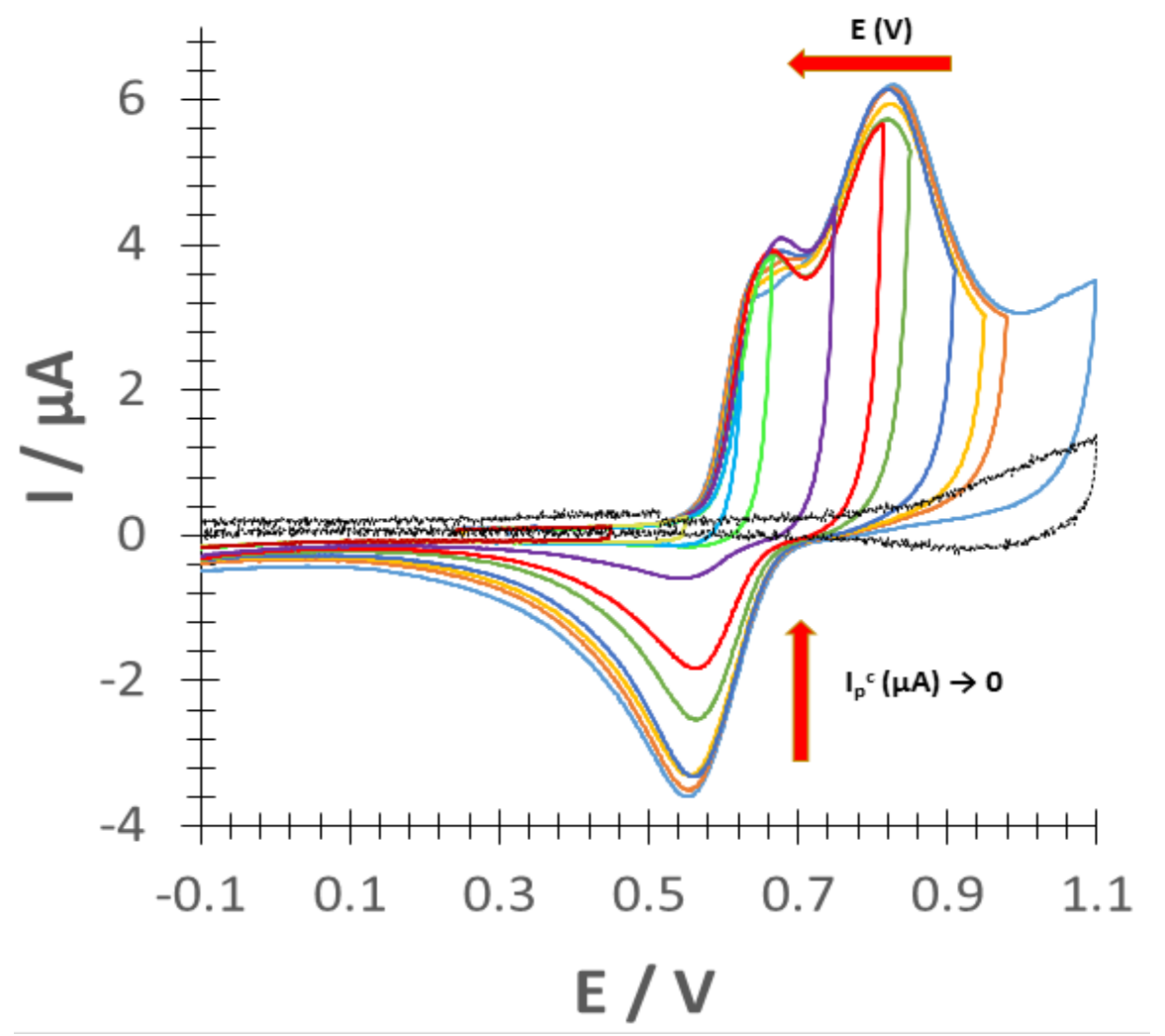

Figura A2.1. Estudio $E_{\lambda}$ de una disolución de acuosa de curcumina [32.5 $\mu \mathrm{M}$ ] a pH $3.576 \pm 0.001$ bajo condiciones experimentales controladas en $\left[\mathrm{ClO}_{4}^{-}\right]=0.068 \mathrm{M}$. ET: $\mathrm{EPC}, \mathrm{ER}: \mathrm{Ag}^{\circ} \mid \mathrm{AgCl}[\mathrm{KCl} 3 \mathrm{M}], \mathrm{EA}$ : Alambre de $\mathrm{Pt}^{\circ} . \mathrm{v}=100 \mathrm{mV} \mathrm{s}^{-1}$. El registro en negro es el blanco. 
Manaia et.al. [46] observa dos procesos de oxidación, mismos que se observan en el voltamperograma a tiempo 0 en la Figura $5 \mathrm{~b}$ del Capitulo 1. Manaia et.al. [46] propone que el primer proceso de oxidación $\left(\mathrm{Ep}^{\mathrm{a}}=0.670 \mathrm{~V}\right.$ en este trabajo, ver Figura A2.1) está asociado a la formación del radical fenóxido, el cual tras una hidrolisis en la posición orto, forma la ortoquinona (Paso I). A valores de potencial más altos Manaia propone un segundo proceso de oxidación (Paso II) tras la hidroxilación formando el grupo ceto $\left(\mathrm{Ep}_{\mathrm{II}}{ }^{\mathrm{a}}=0.813 \mathrm{~V}\right.$ en este trabajo, ver Figura A2.1) y que de acuerdo al estudio $E_{\lambda}$ mostrado en la Figura A2.1 sería un proceso reversible. Esto también coincide con los resultados obtenidos en el Capítulo 3 donde se amortigua el medio a $p \beta-C D=2$. Dado que la curcumina forma un compuesto de inclusion con la $\beta$ $\mathrm{CD}$, la formación del radical fenóxido (Paso I) no puede llevarse a cabo dada la protección de la cavidad de la $\beta-C D$ sobre el grupo -OH involucrando así solo el Paso II (proceso reversible de oxidación a $\mathrm{Ep}_{\mathrm{I}}{ }^{\mathrm{a}}=0.778 \mathrm{~V}$, ver Figura 16b Capítulo 3) del mecanismo propuesto por Manaia y colaboradores. [46]:

En el siguiente esquema se muestra el mecanismo propuesto por Manaia et.al.

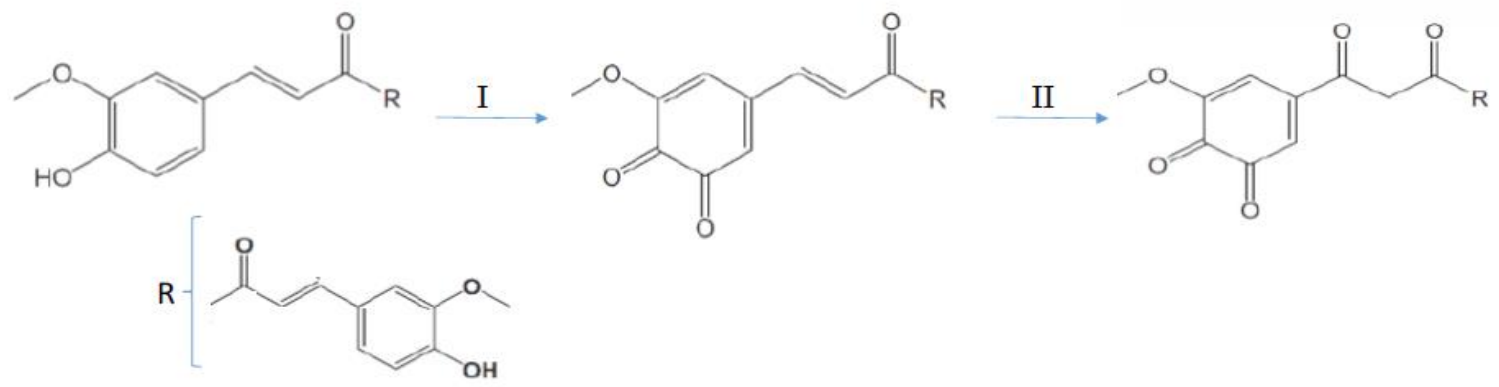

En la Figura 5b del Capítulo 1 se muestra que después de transcurridos 60 minutos el primer proceso de oxidación desaparece con una evidente caída de corriente y cambios en el valor de potencial del proceso de oxidación $E p{ }^{a}{ }^{a}=0.813 \mathrm{~V}$ y del proceso de reducción $E_{p_{1}}{ }^{c}=0.570 \mathrm{~V}$. Estos cambios pueden asociarse según Kallel Trabelsi et.al. [47] a la oxidación del ácido ferúlico, el cual está reportado como uno de los productos de degradación de la curcumina en medio acuoso [16-18] tal como se muestra en el siguiente esquema:<smiles>COC1=CC(/C=C/C(=O)O)=CCC1O</smiles> 


\section{APÉNDICE 3}

\section{Stability QUotiens from Absorbance Data (SQUAD).}

En la década de los setentas surgió un boom en el desarrollo y publicación de programas computacionales para el estudio de los equilibrios en disolución acuosa, específicamente el cálculo de constantes de estabilidad a partir de datos potenciométricos, espectrofotométricos, de potencial por mencionar solo algunos cuantos. De esta manera podemos mencionar el aporte de autores tal como son: Kankare [47] que desarrolló una aproximación usando datos de absorbancia, Motekaitis et.al. [48] que utilizan datos potenciométricos para el cálculo de valores de constantes de acidez usando el programa PKAS el cual es un software escrito en FORTRAN IV. Otro programa que utiliza datos potenciométricos es el de Sabatini et.al [49] que reportan un programa capaz de procesar datos potenciométricos mediante una modificación del método iterativo de Gauss-Newton.

En 1975 Legget y McBryde [50] desarrollan el software SQUAD (Stability QUotiens from Absorbance Data). Es un programa escrito en el lenguaje de programación FORTRAN IV. SQUAD es capaz de calcular simultáneamente o de manera individual varias constantes de estabilidad para cualquier especie formada ya sea por más de un metal o más de un ligando donde el grado de complejación es dependiente del $\mathrm{pH}$.

El algoritmo de SQUAD se basa en la minimización de la suma de residuales al cuadrado de los valores de absorbancia a través de la subrutina REFINE:

$$
\mathrm{U}=\sum_{j=1}^{N}\left[\left(A_{i, k}^{o b s}\right)-\left(A_{i, k}^{c a l}\right)\right]^{2}
$$

Donde: $A^{\text {obs }}$ es la absorbancia experimental y $A^{\text {cal }}$ son los valores de absorbancia calculados con la ley de Lambert-Beer (ver Apéndice 4).

Por tanto, el método reproduce, a partir de datos de absorbancia, constantes de disociación ácida (pka's), constantes de hidrólisis para cationes metálicos, constantes de complejación ( $\mathrm{ML}, \mathrm{ML}_{2}$, etc.) y constantes de complejos polinucleares $\left(\mathrm{M}_{2} \mathrm{~L}_{3}, \mathrm{M}_{2} \mathrm{~L}_{2} \mathrm{H}, \mathrm{M}_{3} \mathrm{~L}_{4}(\mathrm{OH})_{2}\right.$, etc.).

En el siguiente diagrama de flujo se resume la filosofía de SQUAD: 


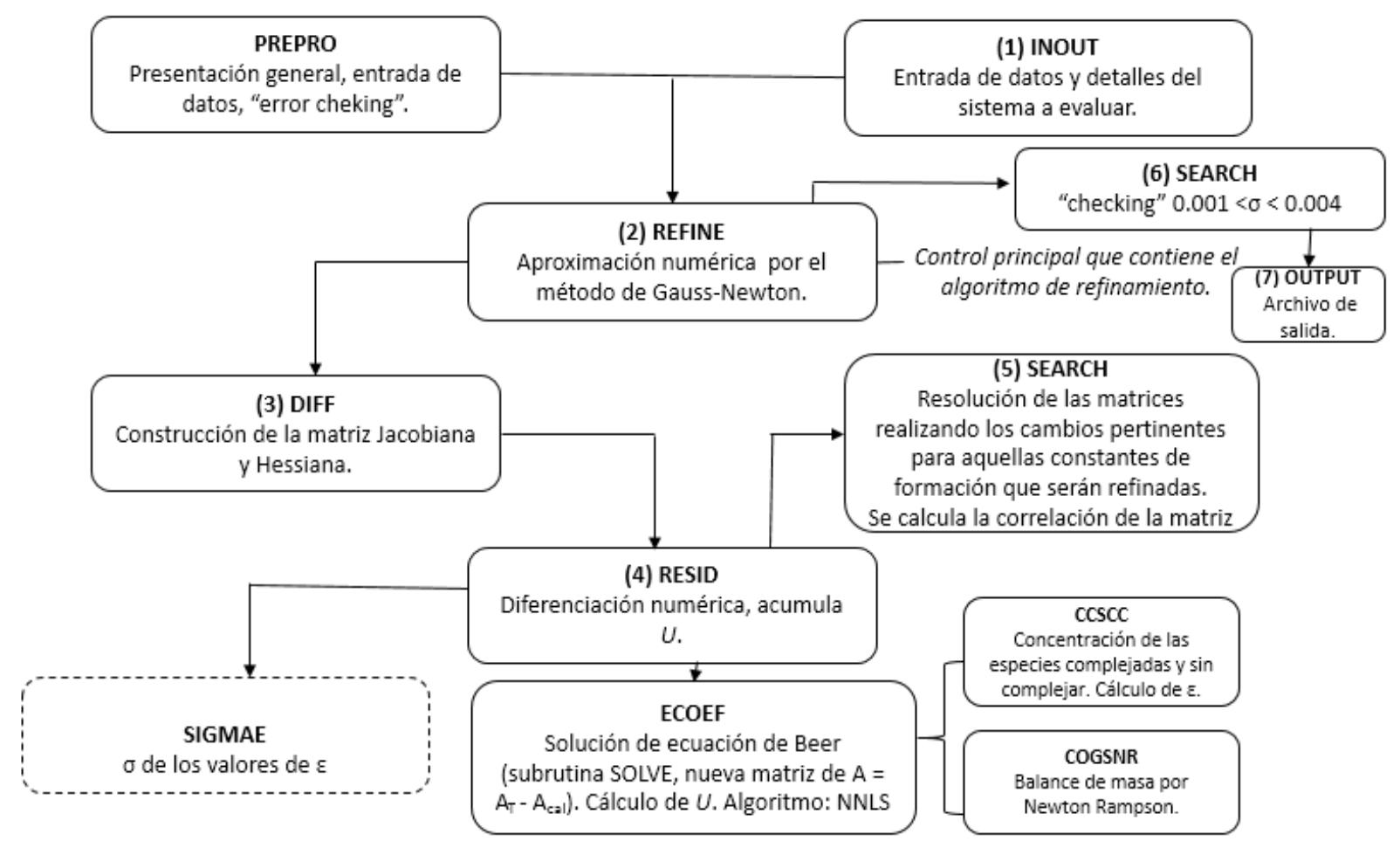

En el siguiente esquema se muestra el archivo de entrada para una corrida de SQUAD:

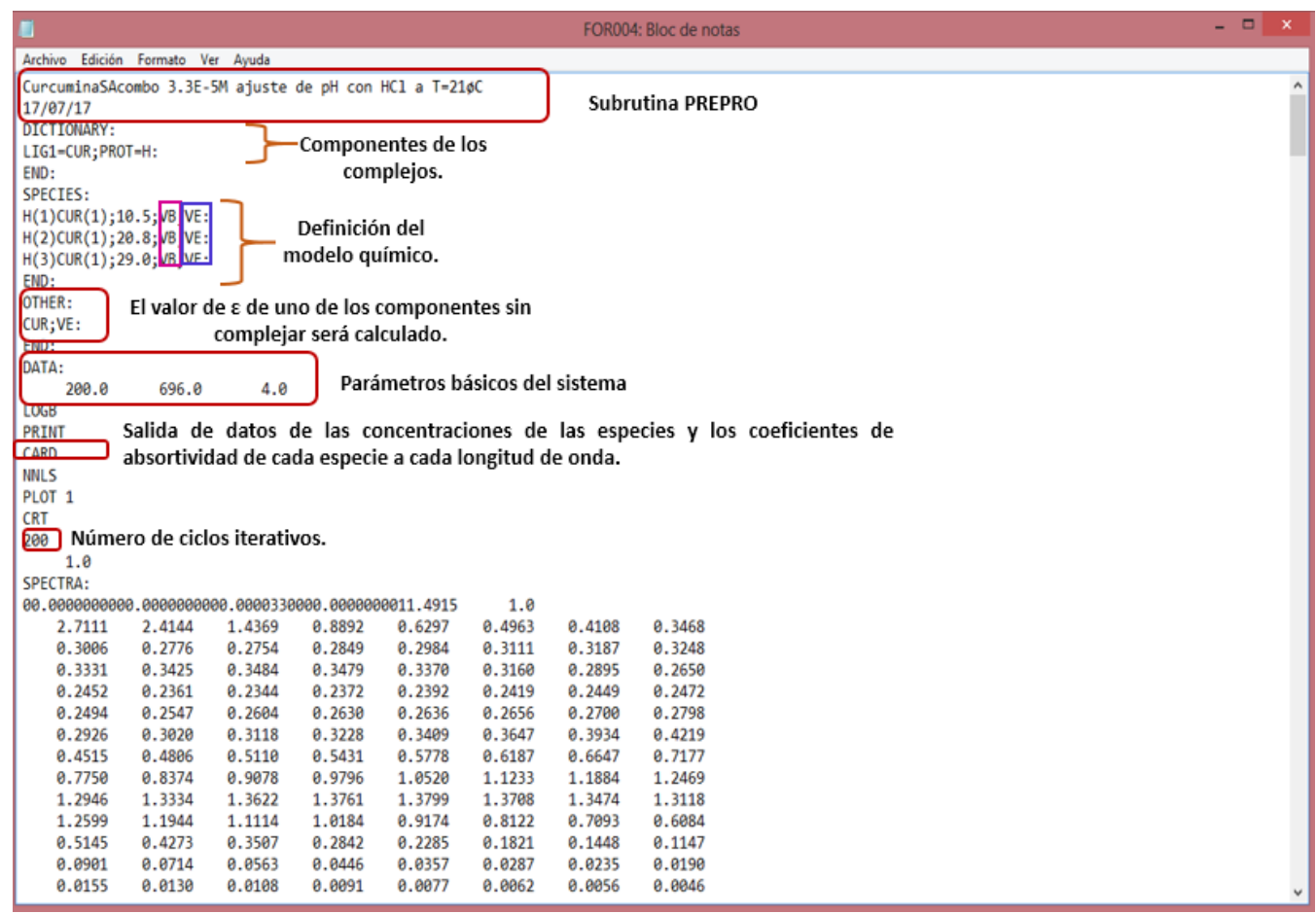


El archivo de salida común para una corrida de SQUAD se muestra a continuación (las siguientes imágenes solo son una muestra de todo el archivo de salida). La información encerrada en rojo muestra los valores 10.9463, 20.4984 y 27.4968 correspondientes a los valores de logaritmo base 10 de las constantes de formación global de protonación refinadas de la curcumina: $\log \beta_{1}, \log \beta_{2}$ y $\log \beta_{3}$.

THE STANDARD DEVIATION IN THE ABSORBANCE DATA IS 1.9856D-02
10.9463
0.0155 SHIFT $=$
$-0.0001 \mathrm{FORH}(1) \mathrm{CUR}(1)$
20.4984
0.0235 SHIFT=
$-0.0005 F O R H(2) C U R(1)$
27.9268
0.0343 SHIFT=
$-0.0011 F 0 R H(3)$ CUR(1)

5 CYCLE(C) CALCULATED

CONVERGENCE ACHIEVED IN 5CYCLES ***

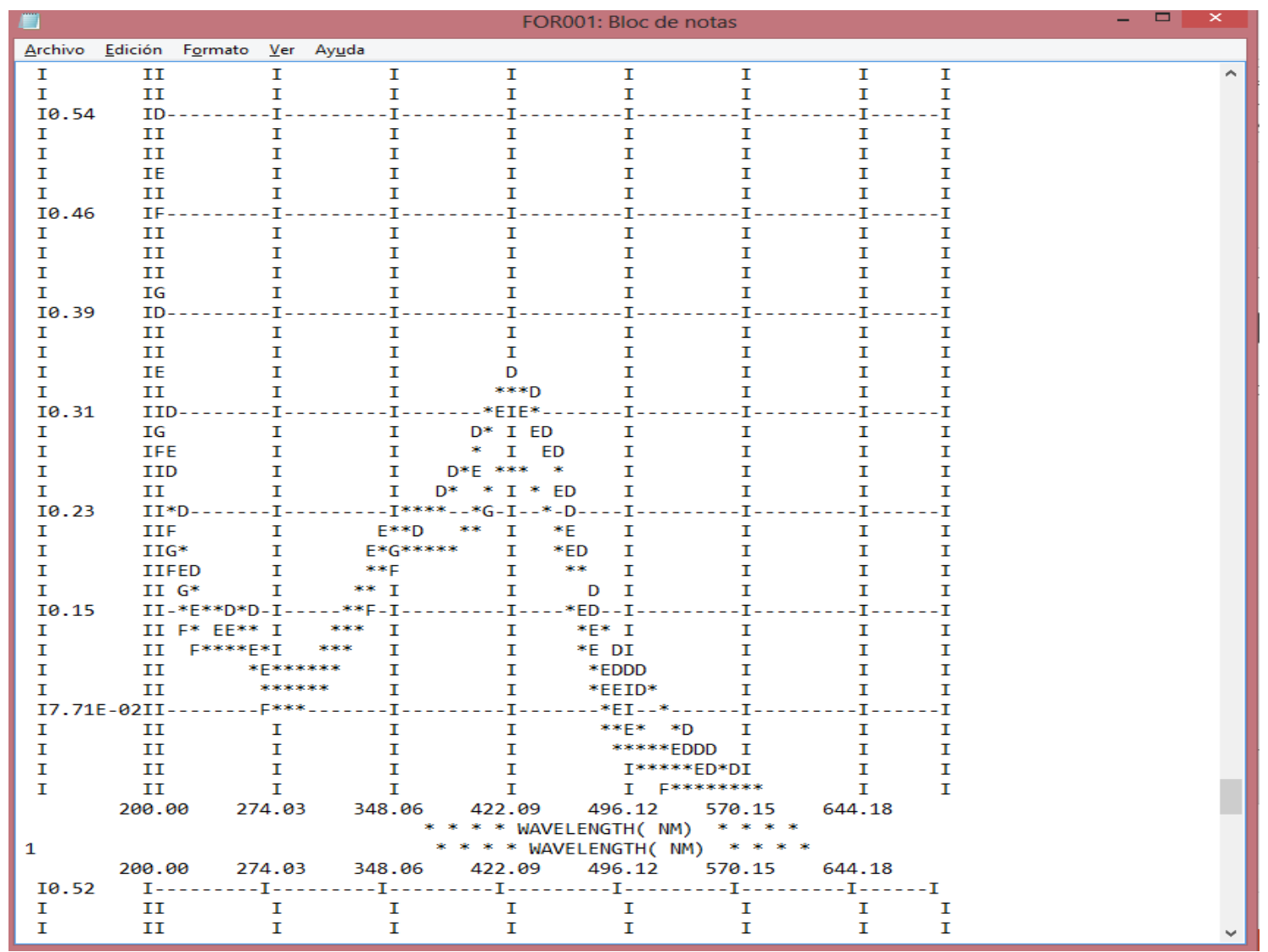




\section{APÉNDICE 4}

\section{Espectrofotometría de UV-Vis}

En muchos tipos de interacción radiación electromagnética-material es útil enfatizar la naturaleza de partícula de la luz como una corriente de fotones o cuantos. La energía de un solo fotón se relaciona con su longitud de onda, frecuencia y número de onda mediante:

$$
\mathrm{E}=h \lambda=\frac{h c}{\lambda}
$$

donde $h$ es la constante de Planck $\left(6.63 \times 10^{-34} \mathrm{~J} \mathrm{~s}\right)$.

La potencia radiante de un haz de radiación es directamente proporcional al número de fotones por segundos.

El espectro electromagnético abarca un gran intervalo de energías (frecuencias) y, por tanto, de longitudes de onda. Las principales divisiones del espectro se muestran a continuación:

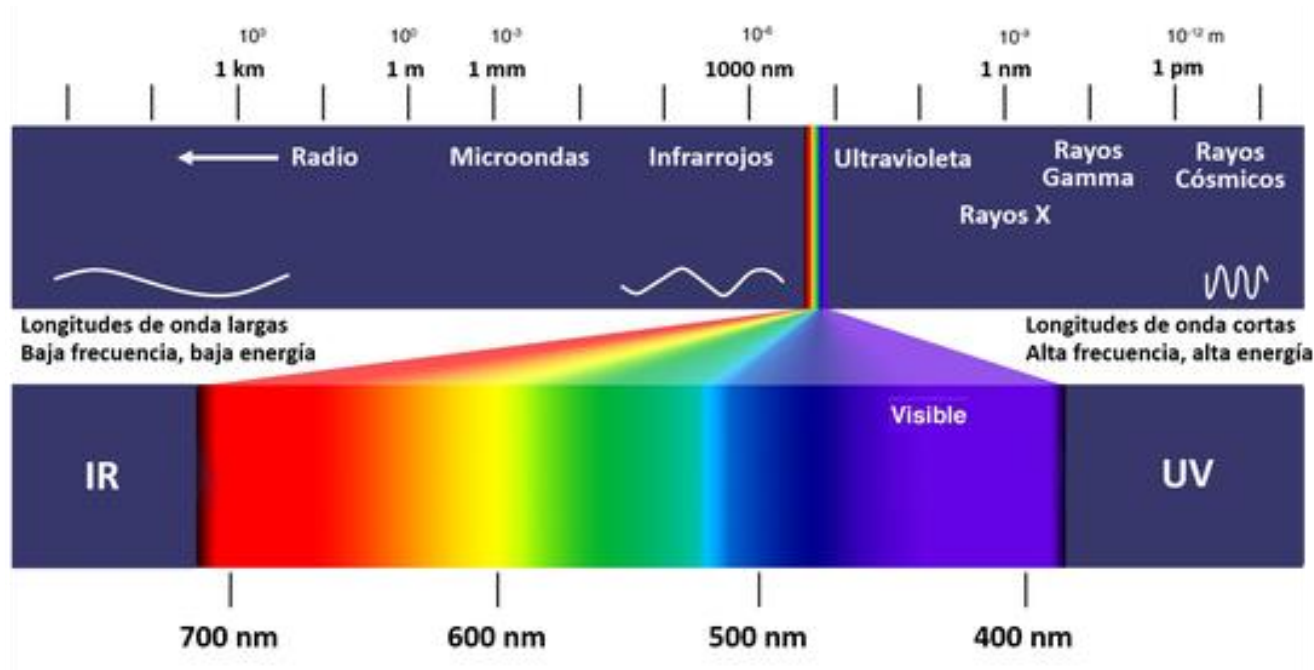

Cada especie molecular es capaz de absorber sus propias frecuencias características de radiación electromagnética. Este proceso de absorción transfiere energía a la molécula y da como resultado una disminución en la intensidad de la radiación electromagnética incidente. Por lo tanto, la absorción de la radiación atenúa el haz de acuerdo con la ley de absorción. La ley de absorción, también conocida como la ley de Beer-Lambert nos indica cuantitativamente cómo la cantidad de atenuación depende de la concentración de las moléculas absorbentes y de la longitud de la trayectoria donde ocurre la absorción. La Figura A4.1 representa la atenuación de un haz paralelo de radiación monocromática a medida que pasa a 
través de una disolución absorbente de densidad $b \mathrm{~cm}$ y concentración $c$ moles por cada litro de disolución.

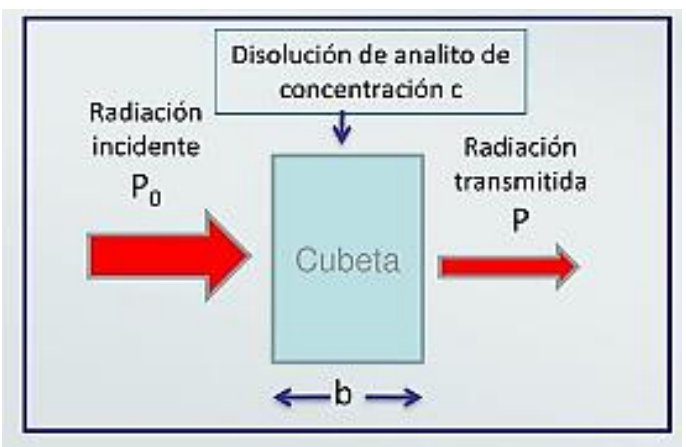

Figura A4.1 Atenuación de un haz de radiación por una disolución absorbente. La longitud de la trayectoria de la disolución absorbente es $b$ y la concentración es $c$.

Debido a las interacciones entre protones y partículas absorbentes la potencia radiante del haz disminuye de $P_{0}$ a $P$. La transmitancia, $T$, de la disolución es la fracción incidente de la radiación transmitida por la disolución. La absorbancia, A, de una disolución está relacionada con la transmitancia de una forma logarítmica, como se muestra a continuación:

$$
A=-\log T=-\log \frac{P}{P_{o}}=\log \frac{P_{o}}{P}=\epsilon b c
$$

donde $\epsilon$ es una constante de proporcionalidad llamada absortividad molar, $b$ la longitud de la cubeta o celda y $c$ es la concentración. 


\section{APÉNDICE 5}

\section{Voltamperometría Cíclica.}

De manera general el término voltametría se refiere a un grupo de métodos electroanalíticos en los cuales se recopila información acerca del analito en una celda electroquímica en función de la señal de excitación (potencial aplicado). De acuerdo a la forma de las ondas de las cuatro señales de excitación más utilizadas en voltametría es como será denominada la técnica voltamperométrica (Figura A5.1). Si el potencial con respecto al tiempo muestra una función de tipo triangular; sobre un electrodo estacionario, estático y en regimen de difusión pura (sin agitar) la técnica recibe el nombre de voltamperometría cíclica.

Nombre

(a)

Barrido

lineal

(b) Impulso
Forma de la señal
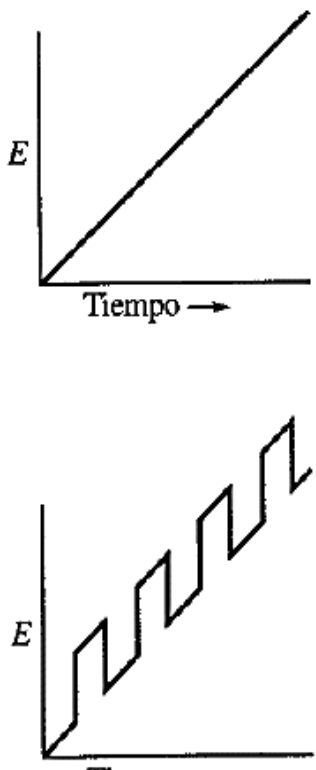

Tipo de voltamperometría

Polarografía

Voltamperometría

de barrido lineal

Polarografía

diferencial

de impulsos 
(c)

Onda cuadrada

(d) Triangular

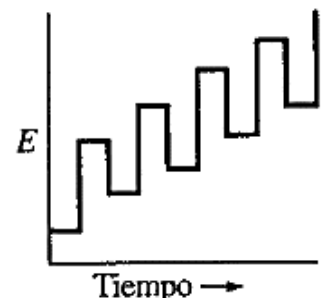

Tiempo $\rightarrow$

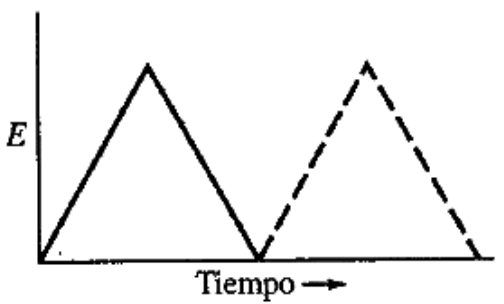

Voltamperometría de onda cuadrada

Voltamperometría cíclica

Figura A5.1 Señales de excitación de voltaje contra tiempo utilizadas en voltametría.

Por ejemplo, en la Figura A5.2 se muestra la respuesta de corriente cuando una disolución que contiene $6 \mathrm{mM}$ de $\mathrm{K}_{3}\left[\mathrm{Fe}(\mathrm{CN})_{6}\right]$ y $\mathrm{KNO}_{3} 1 \mathrm{M}$ se somete a la señal de excitación cíclica mostrada en la Figura A5.1d. El electrodo de trabajo utilizado fue un electrodo estacionario de platino cuidadosamente pulido, y el electrodo de referencia fue un electrodo saturado de calomelanos. En el potencial inicial de $+0.8 \mathrm{~V}$ se observa una pequeña corriente anódica, la cual disminuye inmediatamente a cero a medida que se continúa el barrido. La corriente inicial negativa surge de la oxidación del agua para producir oxígeno (en potenciales más positivos, esta corriente aumenta rápidamente y se hace bastante grande a aproximadamente $+0.9 \mathrm{~V}$ ). No se observa ninguna corriente entre los potenciales de $+0.7 \mathrm{~V} y+0.4 \mathrm{~V}$ debido a que no hay especies oxidables o reducibles presentes en este intervalo de potenciales. Cuando el potencial se hace menos positivo que $+0.4 \mathrm{~V}$, se empieza a desarrollar una corriente catódica (punto B) debido a la reducción del ion hexacianoferrato (III) al ion hexacianoferrato (II). La reacción en el cátodo es, por lo tanto: $\mathrm{Fe}(\mathrm{CN}) 6^{3-}+1 \mathrm{e}^{-}=$ $\mathrm{Fe}(\mathrm{CN}) 6^{4-}$. Un incremento rápido en la corriente ocurre en la región de $\mathrm{B}$ a $\mathrm{D}$ a medida que la concentración en superficie de $\mathrm{Fe}(\mathrm{CN}) 6^{3-}$ se hace cada vez más pequeña. La corriente en el pico está formada por dos componentes. Uno es el avance inicial de la corriente requerido para ajustar la concentración en la superficie del reactivo a su concentración de equilibrio como está dado por la ecuación de Nernst. El segundo es la corriente normal controlada por difusión. La primera corriente decae rápidamente después (puntos $D$ a F) a medida que la capa de difusión se extiende cada vez más lejos de la superficie del electrodo. En el punto $F(-0.15 \mathrm{~V})$, la dirección del barrido se invierte. La corriente, sin embargo, sigue siendo catódica aunque el barrido va hacia 
potenciales más positivos debido a que los potenciales todavía son lo suficientemente negativos como para provocar la reducción del $\mathrm{Fe}(\mathrm{CN}) 6_{6}^{3-}$. A medida que el potencial cambia en dirección positiva, la reducción de $\mathrm{Fe}(\mathrm{CN}) 6^{3-}$, eventualmente se detiene, y la corriente se hace cero y después se vuelve anódica. La corriente anódica es el resultado de la reoxidación del $\mathrm{Fe}(\mathrm{CN})_{6}{ }^{4-}$ que se ha acumulado cerca de la superficie durante el barrido directo. Esta corriente anódica alcanza un pico y posteriormente disminuye a medida que el $\mathrm{Fe}(\mathrm{CN}) 6^{4-}$ acumulado es utilizado por la reacción anódica. Algunas variables importantes en el voltamperograma cíclico son el potencial de pico catódico $E \mathrm{p}_{\mathrm{c}}$, el potencial de pico anódico $E \mathrm{p}_{\mathrm{a}}$. la corriente de pico catódica $\mathrm{I} \mathrm{p}_{\mathrm{c}}$ y la corriente de pico anódica $1 \mathrm{pa}_{\mathrm{a}}$.

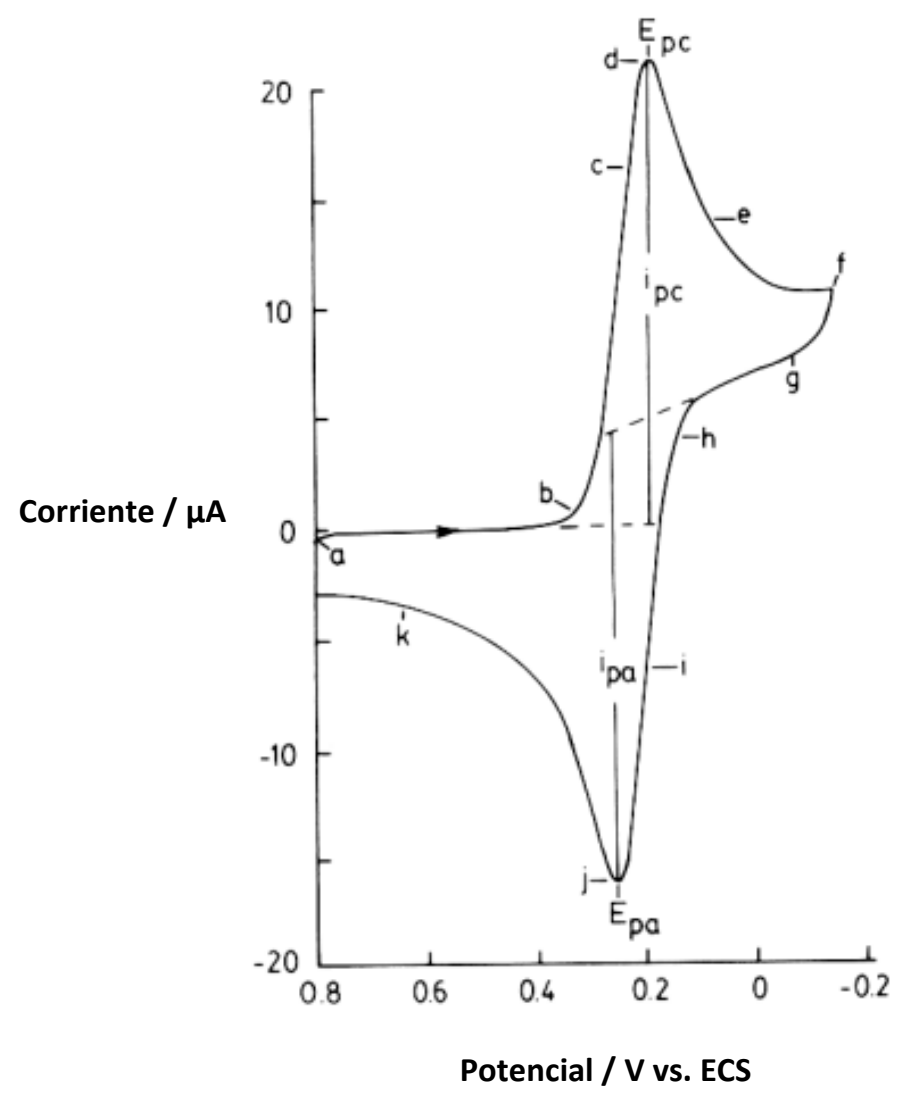

Figura A5.2 Voltamperograma cíclico para una disolución que contiene $6 \mathrm{mM}$ de $\mathrm{K}_{3} \mathrm{Fe}(\mathrm{CN})_{6}$ en $\mathrm{KNO}_{3} 1 \mathrm{M}$. Barrido inicial a $+0.8 \mathrm{~V}$ en dirección negativa. $\mathrm{v}=50 \mathrm{mV} \mathrm{s}^{-1}$. ET: electrodo de platino, ER: SCE.

La celda electroquímica está constituida por tres electrodos sumergidos en una disolución que contiene el analito y también un exceso de un electrolito no reactivo conocido como electrolito soporte. Uno de los tres electrodos es el electrodo de trabajo (ET), cuyo potencial frente a un electrodo de referencia es variado linealmente 
con el tiempo. Las dimensiones del electrodo de trabajo son pequeñas para incrementar su tendencia a polarizarse. El electrodo de referencia $(E R)$ tiene un potencial que permanece constante a lo largo del experimento. El tercer electrodo es un contraelectrodo (CE) o electrodo auxiliar (EA), el cual generalmente es un alambre de platino enrollado cuya función es cerrar el circuito (Figura A5.3).

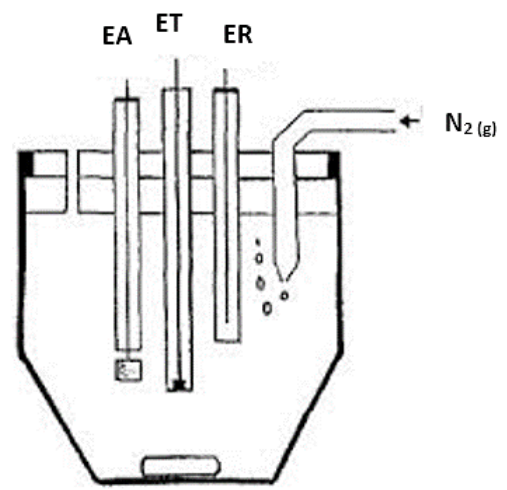

Figura A5.3 Celda con capacidad para tres electrodos. 
ARTÍCULO PUBLICADO 


\title{
New insights on the Chemical Stability of Curcumin in Aqueous Media at Different pH: Influence of the Experimental Conditions
}

\author{
J. Martinez-Guerra', M. Palonar-Pardave", M. Romero-Romo², S. Conona-Avendaĩo', \\ A. Rojas-Hernández", M.T. Ramire-Silva"," \\ ${ }^{1}$ Universidad Autónoma Metropolitana-Iztapalapa, Departamento de Quimica, Av, San Rafael Atlixco \\ \#186, Col. Vicentina, CDMX. C.P. 09340, México \\ ${ }^{2}$ Universidad Autónoma Metropolitana-Azcapotzalco, Departamento de Materiales, Av. San Pablo \\ 180 Col. Reynosa-Tamaulipas, CDMX, C.P. 02200, México. \\ "E-mail: mtrs218@xanumuam.mx, mepp@icorreo azc uammx
}

Received: 18 January 2019 / Accepted: 18 March 2019 / Published: 10 May 2019

From spectrophotometric and electrochemical measurements recorded in curcumin aqueous solutions at different $\mathrm{pH}$ values, both the stability and the acidity constants of curcumin were evaluated. It was found that contrary to hitherto acceptances, curcumin becomes stable at basic $\mathrm{pH}$ values while at acid ones curcumin rate of degradation is around 20 times higher than in neutral or basic conditions. Notwithstanding, by controlling the experimental conditions the degradation rate of curcumin can be diminished even at acid and neutral conditions. Our results show that curcumin has $3 \mathrm{pKa}$ values, namely: $7.428=0.015,9.552=0.024$ and $10.946 \pm 0.034$, and for the first time, the molar absorptivity coefficients of the curcumin species in aqueous media: $\mathrm{H}_{3} \mathrm{Cur}, \mathrm{H}_{2} \mathrm{Cur}^{-}, \mathrm{HCur}^{2-}$ and $\mathrm{Cur}^{3-}$, were reported as a function of the wavelength.

Keywords: Curcumin; stability; aqueous solution; $\mathrm{pH}$; $\mathrm{pKa}$

\section{INTRODUCTION}

Curcumin [1,7-bis(4-hydroxy-3-methoxyphenyl)-1,6-heptadiene-3,5-dione] the main component of turmeric curcumin, also known as curcuma, is constituted by demethoxycurcumin, the bisdemethoxycurcumin and the cyclocurcumin. It is found in the roots of the Curcuma Longa L. plant [1]. The curcumin is a phenol compound with antiinflamatory $[1,2]$, anticarcinogenic [1, 3-5], antioxidant [1, 3, 6-8] and antiviral [9-13], properties that have been amply reported. Likewise, is used as coloring and spice in the textile and food industries [14], respectively. However, its low solubility in water gives rise to a low biodisponibility, which has hindered its potential application in the 
ACTA DE EXAMEN PROFESIONAL 
ACTADE DISERTA

UNIVERSIDAD AUTONOMA METROPOLITANA

Matricula. 2141801250

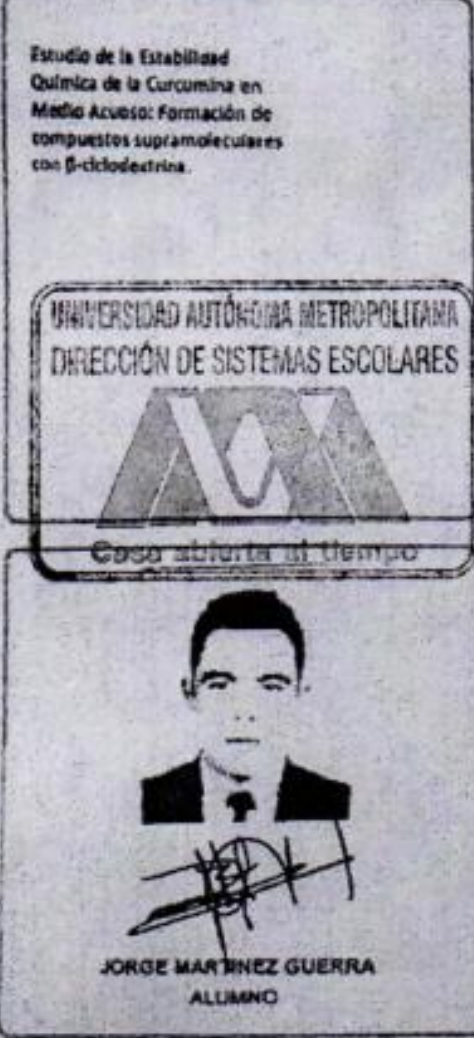

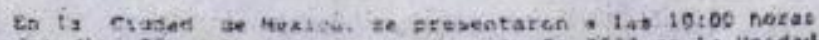

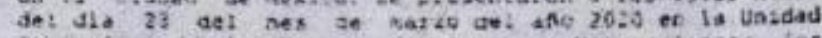

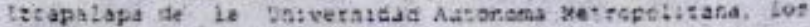
suscrites sloebros del jurace.

\begin{abstract}
Dhh. GREKERLA HOA MORALES
ORA. FATRICIA BALOTERAS MERLAMOEZ

DR. JOFGE IVAR ALDANA GOHZALEZ

DR. CARLOS AUDRFS GNLAN VIDAL

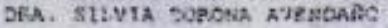

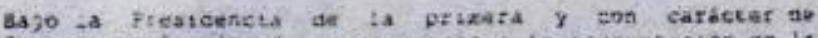

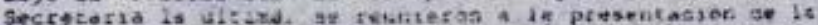
Daxectacior pub.tct Gage dencainastori aparect of mergen.

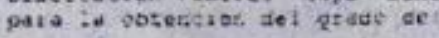

Doctork s.A ctructas isuimica?

SE: JORGE MARTINEZ GUEGSh

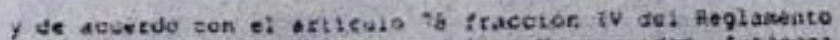
de Estudios Superiores ae id universidaro hatonoses Metropolitand, los pienbres del jutado resolvieron:

\title{
AiPQTAF
}

Aeto continco, la presidenta oel jurade cabunzed at

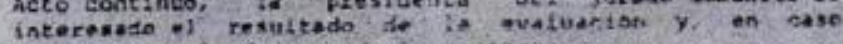
aprobstorio. le tue tomads is protesta.

OIRECTOR DE LA DNYSION OE CQI

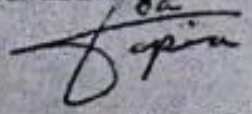

DR JESUS NLBERTO OCHOA TAPL
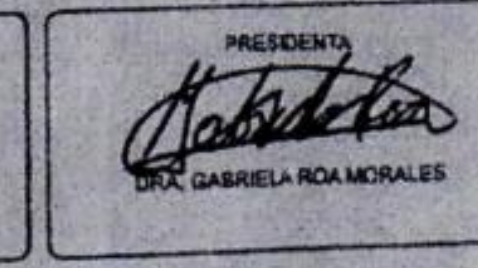
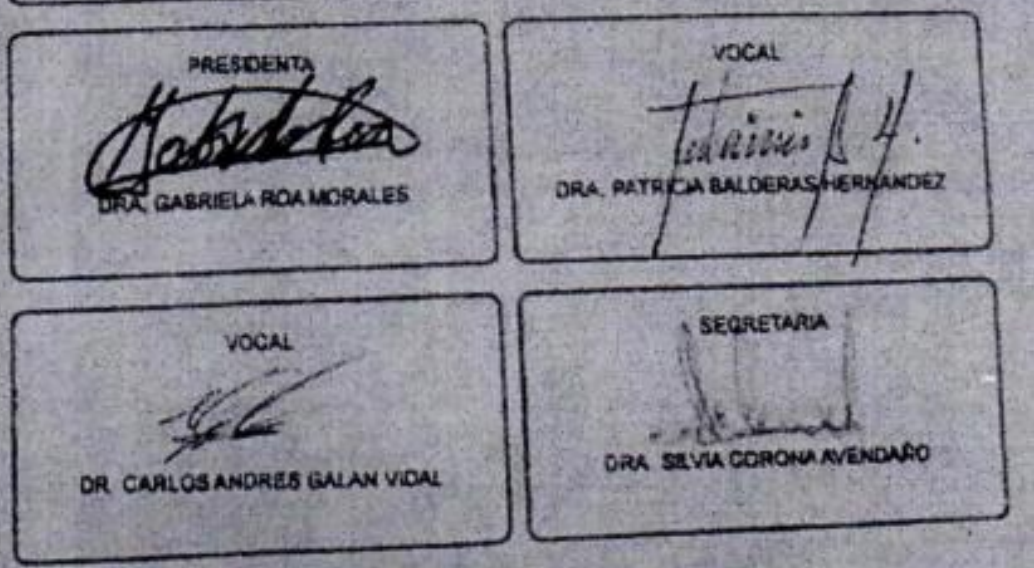